\title{
Medidas invariantes para aplicações unimodais
}

Belmiro Galo da Silva

\author{
DISSERTAÇÃO APRESENTADA \\ AO \\ Instituto de Matemática e Estatística \\ DA \\ Universidade De SÃo Paulo \\ PARA \\ OBTENÇÃO DO TÍTULO \\ $\mathrm{DE}$ \\ Mestre em CiÊnCIAS \\ Programa: Matemática aplicada \\ Orientador: Prof. Dr. Edson Vargas
}

Durante o desenvolvimento deste trabalho o autor recebeu auxílio financeiro do $\mathrm{CNPq}$

São Paulo, Fevereiro de 2014 



\section{Medidas invariantes \\ para aplicações unimodais}

Esta é a versão original da dissertação elaborada pelo candidato Belmiro Galo da Silva, tal como submetida à Comissão Julgadora. 



\section{Medidas invariantes para aplicações unimodais}

Esta versão da dissertação contém as correções e alterações sugeridas pela Comissão Julgadora durante a defesa da versão original do trabalho, realizada em 21/02/2014. Uma cópia da versão original está disponível no Instituto de Matemática e Estatística da Universidade de São Paulo.

Comissão Julgadora:

- Prof. Dr. Edson Vargas - IME-USP

- Prof. Dr. Pedro Antônio Santoro Salomão - IME-USP

- Prof. Dr. Daniel Smania Brandão - ICMC-USP 



\section{Agradecimentos}

A Juan Letelier, Mark Gannon, Pablo Guarino, Simon Lloyd, Tertuliano Franco, André Alves, Arlane Silva, Bruno de Castro, Eliardo Costa e Tiago Estrela por me ajudarem diretamente neste trabalho.

A Bruna Cassol dos Santos, companheira incondicional.

Aos amigos de longas datas e aos novos colegas e amigos que surgiram ao longo do curso por contribuirem durante esse período, sendo muitas vezes com um sorriso, um abraço ou palavras de incentivo.

A minha família: minha mãe Deborah, minha irmã Marcella, meu paidrasto Danilo e ao pequeno Davi.

A Alfredo Jorge Aragona, Orlando Lopes, Rodrigo Bissacot e aos demais professores e funcionários do IME-USP que contribuíram com a minha formação ao longo deste curso.

Não podia deixar de agradecer aos meus professores do IM-UFBA, em especial, Carlos Bahiano e Samuel Silva por contribuirem para a minha formação matemática e crescimento acadêmico.

Ao meu orientador, Edson Vargas pela escolha de um tema que me deixou bastante entusiasmado pela pesquisa na área, além da sua paciência, ajuda e disponibilidade em todas as fases deste trabalho. 


\section{Resumo}

GALO, B. Medidas invariantes para aplicações unimodais. 2014. $84 \mathrm{f}$. Tese (Mestrado) Instituto de Matemática e Estatística, Universidade de São Paulo, São Paulo, 2014.

Neste trabalho estudamos medidas invariantes para aplicações unimodais. Estamos especialmente interessados em detectar as situações que levam uma aplicação unimodal a não possuir uma medida piac, ou seja, uma medida de probabilidade invariante e absolutamente contínua em relação à medida de Lebesgue. Mostramos que a ordem do ponto crítico e a sua capacidade de recorrência são os fatores mais relevantes nesta questão. Os valores das derivadas da aplicação nos pontos periódicos tem uma influência menor, mas suficiente para garantir que numa mesma classe de conjugação topológica podem existir duas aplicações unimodais com ponto crítico de mesma ordem, sendo que uma delas possui medida piac e a outra não possui. A capacidade de recorrência do ponto crítico, talvez o principal fator nesta questão, depende de aspectos combinatórios bem sofisticados. As ferramentas principais para analisar estes aspectos envolvem os conceitos de tempos de corte e de aplicações kneading.

A existência ou não de medidas piac é uma propriedade de natureza métrica, e por isto, é necessário que tenhamos controle de como os iterados da aplicação unimodal distorcem a medida de Lebesgue. Então precisamos usar ferramentas de controle de distorção que incluem principalmente os Princípios de Koebe. Um ponto culminante deste trabalho diz respeito a relação entre existência de mediada piac e existência de atratores selvagens, isto é: atratores métricos que não são atratores topológicos e vice versa. Usamos aqui um argumento probabilístico de rara beleza.

Palavras-chave: aplicação unimodal, aplicação kneading, medida invariante, atrator. 


\section{Abstract}

GALO, B. Invariant measures for unimodal maps. 2014. 84 f. Tese (Mestrado) - Instituto de Matemática e Estatística, Universidade de São Paulo, São Paulo, 2014.

In this work we study invariant measures for unimodal maps. We are especially interested in detecting situations that cause a unimodal map not to have a piac measure, i.e., a measure that is Probability Invariant and Absolutely Continuous with respect to Lebesgue measure. We show that the order of the critical point and its capacity for recurrence are the most relevant factors in this matter. The values of the derivatives of the map at periodic points have a small influence, but enough to ensure that within a single class of topological conjugacy, there can be two unimodal maps with critical points of the same order, one of which has a piac measure and the other does not. The recurrence capacity of the critical point depends on very sophisticated combinatorial aspects and is probably the main factor in this issue. The main tools to analyze these aspects involve the concepts of cutting times and kneading maps.

The existence of piac measures is a property of metric nature, and for this reason we need to have control of how iterations of the unimodal map distort the Lebesgue measure. We therefore need to use distortion control tools, including especially the Principles of Koebe. A culmination of this work concerns the relationship between existence of piac measures and the existence of wild attractors, i.e., metric attractors that not are topological attractors. Here we use a probabilistic argument of rare beauty.

Keywords: Unimodal map, kneading map, invariant measure, attractor. 


\section{Sumário}

$\begin{array}{ll}\text { Lista de Abreviaturas } & \text { ix }\end{array}$

Lista de Símbolos $\quad$ xi

Lista de Figuras $\quad$ xiii

Introdução $\quad$ Xv

1 Resultados Principais $\quad 1$

2 Ferramentas Topológicas e Combinatórias $\quad 5$

2.1 Itinerários, equivalência combinatória e topológica . . . . . . . . . . . 5

2.2 Tempos de Corte e a Aplicação Kneading . . . . . . . . . . . . . . . . . . 7

2.3 Aplicação Kneading e a sequência kneading . . . . . . . . . . . . . . . . 10

3 Ferramentas Métricas 13

3.1 Derivada Schwarziana . . . . . . . . . . . . . . . . . . . . . . 13

3.2 Distorção e a razão cruzada . . . . . . . . . . . . . . . . . . . 15

3.3 Princípio de Koebe . . . . . . . . . . . . . . . . . . . . . . 17

3.4 Aplicações de Markov . . . . . . . . . . . . . . . . . . . . . 20

3.5 Resultados da Teoria da medida e ergódica . . . . . . . . . . . . . . . . 21

4 Conjugações topológicas e a propriedade métrica $\quad 23$

4.1 Construindo a medida . . . . . . . . . . . . . . . . . . . 23

4.2 Retorno Sela-nó . . . . . . . . . . . . . . . . . . . . . . . . 28

4.3 Construção do algoritmo da sequência kneading . . . . . . . . . . . . . . . 33

4.4 Demonstração do Teorema A . . . . . . . . . . . . . . . . . . 36

5 Conjunto de Cantor Absorvente $\quad 41$

5.1 A Combinatória da aplicação de Fibonacci . . . . . . . . . . . . . . . . . . . . 42

5.2 Estimativas . . . . . . . . . . . . . . . . . . . 44

5.3 Ferramentas Probabilísticas e Ergódicas . . . . . . . . . . . . . . . 53

5.4 Demonstração do Teorema B . . . . . . . . . . . . . . . . . . . . 58

$\begin{array}{ll}\text { Referências Bibliográficas } & 61\end{array}$ 
viii SUMÁRIO 


\section{Lista de Abreviaturas}

piac Medida de probabilidade invariante absolutamente contínua

q.t.p. Quase todo ponto

q.c Quase certamente 


\title{
Lista de Símbolos
}

\author{
c Ponto crítico \\ $S_{k} \quad k$-ésimo tempo de corte \\ $z_{k} \quad k$-ésimo ponto pré-crítico mais próximo de $c$ \\ $\tau_{k} \quad k$-ésimo tempo de co-corte \\ $\ell \quad$ Ordem do ponto crítico \\ $Q \quad$ Aplicação Kneading \\ $\hat{x} \quad$ Involução de $x: f(\hat{x})=f(x)$
}




\section{Lista de Figuras}

1.1 Uma aplicação tenda . . . . . . . . . . . . . . . . . . . . . . . 1

1.2 Uma aplicação quadrática . . . . . . . . . . . . . . . . . . . 1

2.1 Aplicação $f^{S_{k}}$ para construção da Kneading . . . . . . . . . . . . . . . . 8

2.2 Aplicação $f^{S_{k}}$ para $f^{S_{k-1}}(c) \in\left(z_{Q(k)-1}, z_{Q(k)}\right) \cup\left(\hat{z}_{Q(k)}, \hat{z}_{Q(k)-1}\right) \ldots \ldots \ldots$

4.1 Aplicação induzida $F$ para a aplicação de Fibonacci . . . . . . . . . . . . . . 24



4.3 Aplicação $f^{n}$ para existência do $\gamma>0 \ldots \ldots \ldots \ldots \ldots \ldots$

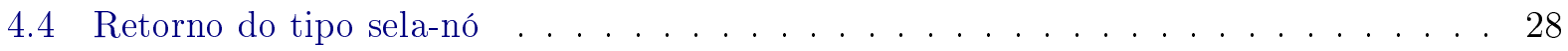

4.5 Aplicação induzida $F$ para $\nu(c)=1011011011011111 \ldots \ldots \ldots$. . . . . . . . . . 34

5.1 Aplicação $f^{S_{n+1}}$ para $d_{n} \in A_{n-1} \ldots \ldots \ldots \ldots \ldots \ldots \ldots$

5.2 Aplicação $f^{S_{n}}$ para estimativas . . . . . . . . . . . . . . . . 44



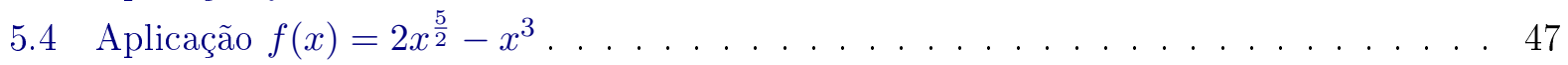

5.5 Aplicação $f^{S_{n}-1}$ para o cálculo de $\left|D f^{S_{n}}(f(a))\right| \ldots \ldots \ldots \ldots$. . . . . . . . 48

5.6 Aplicação $f^{S_{m}-1}$ para obter cota por baixo de $\left|D f^{S_{m}}(f(c))\right| \ldots \ldots \ldots \ldots$. . . . . 50

5.7 Aplicação $f^{S_{m}-1}$ para obter cota por cima de $\left|D f^{S_{m}}(f(c))\right| \ldots \ldots \ldots \ldots$. . . . . 51

5.8 A torre para a aplicação de Fibonacci . . . . . . . . . . . . . . 54 


\section{Introdução}

O objetivo principal deste trabalho é discutir medidas invariantes para sistemas dinâmicos unidimensionais, em particular, para aplicações contínuas, de um intervalo fechado $I$ nele próprio, unimodais. Uma parte importante na compreensão de um sistema dinâmico consiste na compreensão das suas medidas invariantes e suas propriedades. Aplicações unimodais sempre possuem muitas medidas invariantes mas, muitas delas não são interessantes. Dentre as medidas invariantes que são interessantes está a medida de probabilidade invariante e absolutamente contínua em relação à medida de Lebesgue.

No Capítulo 1 introduzimos alguns conceitos básicos e os enunciados dos resultados principais deste trabalho.

No Capítulo 2 faremos estudo sobre diversas ferramentas topológicas e combinatórias no contexto de dinâmica unidimensional. Veremos a importância da dinâmica simbólica para aplicações unimodais e nos familiarizaremos com as aplicações kneading e as sequências kneading.

No Capítulo 3 faremos um apanhado sobre diversos conceitos básicos de natureza métrica que desempenharão um papel importante no trabalho como: a derivada de Schwarz, distorção da razão cruzada, princípio do mínimo, princípio de Koebe e aplicações de Markov.

No Capítulo 4 nosso objetivo principal é provar o seguinte resultado:

Teorema A [Henk Bruin, 1998]. Existem aplicaçôes unimodais $q_{\alpha}$ que possuem uma medida piac e são topologicamente conjugadas a aplicaçôes unimodais $f_{\beta}$ que não possuem medida piac.

Para provarmos, construiremos a medida invariante, nos desvencilhando do ponto crítico, através das aplicações induzidas de Markov. Perceberemos a impotância de possuirmos aplicações com a propriedade de ser ramo-longo, com retornos do tipo sela-nó e que a derivada no ponto fixo assuma valores diferentes para cada uma das aplicações.

No Capítulo 5 estaremos interessados no estudo da medida de Lebesgue dos conjuntos de Cantor minimais de funções unimodais, em especial, estaremos buscando condições de existência para conjuntos de Cantor minimais de medida Lebesgue nula cuja bacia tem medida de Lebesgue total.

As aplicações de Fibonacci desempenharão um papel importante neste contexto, por isso observaremos o comportamento combinatorial desta aplicação e algumas de suas propriedades, pois esta classe de família, com uma ordem crítica suficientemente alta, vai nos permitir concluir o seguinte resultado:

Teorema B.[H. Bruin, G. Keller, T. Nowicki e S. van Strien, 1996] Se $g_{\gamma}$ é aplicação de Fibonacci e $\ell$ é suficientemente grande, o conjunto $\omega$-limite do seu ponto crítico é um conjunto de Cantor minimal de medida Lebesgue nula cuja bacia tem medida de Lebesgue total. 


\section{Capítulo 1}

\section{Resultados Principais}

Este capítulo tem por objetivo introduzir alguns conceitos básicos e os resultados principais deste trabalho. O assunto a ser tratado se insere no contexto de dinâmica unidimensional e diz respeito a iterações de aplicações unimodais. Uma aplicação contínua de um intervalo fechado $I$ nele próprio, $f: I \rightarrow I$, é chamada de aplicação unimodal quando possui um único extremo local no interior de $I$. Este ponto pode ser um ponto de máximo ou de mínimo local e é denotado pela letra $c$. Quando $f$ é de classe $C^{1}$, este extremo local é um ponto crítico, o qual assumiremos ser o único ponto crítico de $f$. Assumimos também, sem perda de generalidade, que $f$ aplica o bordo de $I$ em um único ponto do bordo de $I$.

As aplicaçôes tendas $T_{\alpha}(x)=\min \{\alpha x, \alpha(1-x)\}$ e as aplicações quadráticas $q_{\alpha}(x)=2 \alpha x(1-x)$, onde $x \in I=[0,1]$ e $\alpha$ é um parâmetro que varia em $] 0,2]$, são os exemplos mais populares de aplicação unimodal (veja as figuras abaixo).

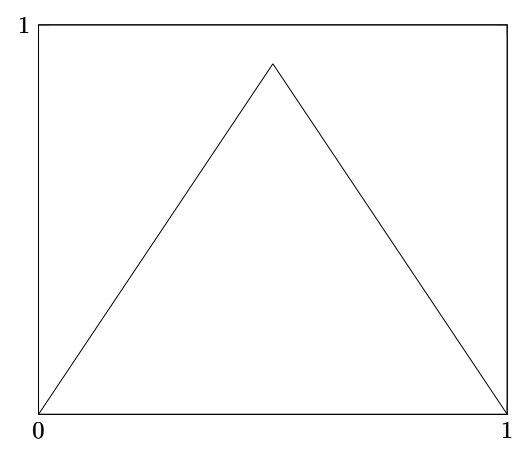

Figura 1.1: Uma aplicação tenda

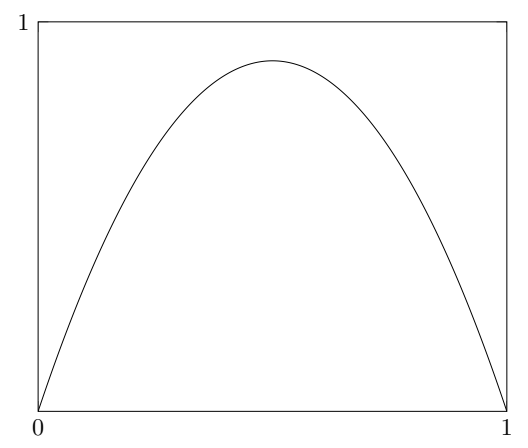

Figura 1.2: Uma aplicação quadrática

O extremo local (ou ponto crítico) $c \in I$ de uma aplicação unimodal $f$ desempenha um papel fundamental para a descrição das propriedades topológicas e métricas da sua dinâmica. A ordem deste ponto crítico, a maneira pela a qual a sua órbita positiva está ordenada no intervalo e o seu grau de recorrência são de grande relevância para aspectos topológicos e métricos desta dinâmica. Dadas duas aplicações unimodais $f$ e $g$, um homeomorfismo $h$ do intervalo $I$ tal que $h \circ f=g \circ h$ é chamado de conjugação topológica entre $f$ e $g$. Neste caso, dizemos que $f$ e $g$ são topologicamente conjugadas. Uma conjugação topológica preserva as propriedades topológicas da dinâmica mas, muitas vezes não preserva a medida de Lebesgue de conjuntos invariantes, por exemplo. Também não preservam outras propriedades métricas como a propriedade de possuir uma medida de probabilidade invariante e absolutamente contínua em relação à medida de Lebesgue. Este é um dos temas que exploramos neste trabalho.

Dizemos que o extremo local (ou ponto crítico) $c$ de uma aplicação unimodal $f$ tem ordem $\ell \geq 1$, 
se existem constantes $0<O_{1} \leq O_{2}$ tais que para todo $x \in I$ vale que:

$$
O_{1}|x-c|^{\ell} \leq|f(x)-f(c)| \leq O_{2}|x-c|^{\ell} .
$$

Observamos que se $f$ é de classe $C^{2}$, então a razão $O_{2} / O_{1}$ pode ser escolhida tão próxima de 1 quanto se queira, para isto é necessário escolher $x$ suficientemente próximo de $c$.

Uma medida $\mu$ definida na $\sigma$-álgebra de Borel do intervalo $I$ é dita invariante por uma aplicação unimodal $f$ se $\mu(A)=\mu\left(f^{-1}(A)\right)$, para todo boreliano $A \subset I$. Se $\mu(I)=1$ dizemos que $\mu$ é uma medida de probabilidade, por brevidade, dizemos apenas probabilidade.

Uma aplicação unimodal sempre possui muitas probabilidades invariantes mas, muitas delas não são interessantes. Dentre as medidas invariantes que são interessantes está a medida de probabilidade invariante e absolutamente contínua em relação à medida de Lebesgue às quais nos referimos como medidas piac. Estas são as probabilidades invariantes que são positivas em todos os borelianos que tem medida de Lebesgue positiva. Por exemplo, uma aplicação tenda $T_{\alpha}$, para $\alpha>1$, sempre possui uma medida piac.

Por outro lado, existem aplicações quadráticas que são topologicamente conjugadas à aplicações tendas mas que não possuem medidas piac. Ainda mais surpreendente é que existem aplicações unimodais $f$ e $g$ analíticas, com ponto crítico de ordem 2, topologicamente conjugadas tais que $f$ possui uma medida piac e $g$ não possui [Bru98]. Na verdade estas aplicações $f$ e $g$ podem ser escolhidas nas famílias $f_{\beta}(x)=\beta \sin (\pi x)$ e $q_{\alpha}(x)=2 \alpha x(1-x)$, respectivamente. Este é o conteúdo do teorema a seguir.

Teorema A [Henk Bruin, 1998]. Existem aplicaçôes unimodais $q_{\alpha}$ que possuem uma medida piac e são topologicamente conjugadas a aplicaçôes unimodais $f_{\beta}$ que não possuem medida piac.

Uma conjugação topológica entre aplicações unimodais como no teorema A aplica um conjunto de medida de Lebesgue positiva em um conjunto com medida de Lebesgue nula. De acordo com este teorema, existem outros fatores além da ordem do ponto crítico e a classe de conjugação topológica que estão relacionados com a existência ou não de uma medida piac. Veremos que os auto valores da derivada da aplicação unimodal nos seus pontos periódicos também tem influência nesta propriedade. O tipo topológico das aplicações unimodais que construímos para provar o teorema acima é muito especial, mesmo fazendo simulações muito precisas dão a impressão que estas aplicações possuem pontos periódicos atratores. Na verdade elas possuem o que chamamos de "retornos selas-nó" que são muito longos e em particular os seus pontos críticos são fortemente recorrentes. Mais a frente neste trabalho formulamos estes aspectos de modo mais preciso.

Dada uma aplicação unimodal $f$ de um intervalo $I$, consideramos a aplicação $\tau: I \rightarrow I$ tal que $f \circ \tau(x)=f(x)$ e, para $x \neq c$, temos que $\tau(x) \neq x$. Se $c$ é recorrente, definimos a sequência de inteiros, chamados de tempos de primeira aproximação, $n_{1}<n_{2}<n_{3}<\ldots$ indutivamente, colocando $n_{1}=1, n_{2}=2$ e escolhendo $n_{i}$ como sendo o menor inteiro tal que $f^{n_{i}}(c)$ esteja entre $f^{n_{i-1}}(c)$ e $\tau\left(f^{n_{i-1}}(c)\right)$. Quanto menores forem os tempos de primeira aproximação mais fortemente recorrente será o ponto $c$. Existem aplicações unimodais tais que os tempos de primeira aproximação são $n_{1}=1, n_{2}=2, n_{3}=2^{2}, \ldots, n_{i+1}=2^{i}, \ldots$ são chamadas de aplicações de Feigenbaum-Tresser. Aquelas em que estes tempos são os números de Fibonacci, a saber $n_{1}=1, n_{2}=2$ e $n_{i+1}=n_{i}+n_{i-1}$, são chamadas de aplicações de Fibonacci. Dentre as aplicações unimodais que não possuem órbita periódica atratora, as aplicações de Feigenbaum-Tresser são as que tem o extremo local $c$ mais fortemente recorrente. A aplicação de Fibonacci são as que tem o extremo local $c$ mais fortemente recorrente dentre as que são topologicamente conjugadas com uma tenda sem intervalo periódico. O conjunto $\omega$-limite de $c$, tanto para as aplicações de Feigenbaum-Tresser como para as aplicações de Fibonacci são conjuntos de Cantor invariantes.

Seja $\Lambda \subseteq I$, um conjunto compacto e invariante por uma aplicação unimodal $f$. Definimos a bacia de $\Lambda$ como sendo o conjunto: $\mathcal{B}(\Lambda)=\{x \in I: \omega(x)=\Lambda\}$.

Para as aplicações unimodais $f=q_{\alpha}$ e $g=f_{\beta}$ do teorema A, $c=1 / 2$ é o ponto crítico e os intervalos $\Lambda_{f}=\left[f^{2}(c), f(c)\right]$ e $\Lambda_{g}=\left[g^{2}(c), g(c)\right]$ são compactos invariantes e transitivos para $f$ 
e $g$, respectivamente. Além disto, as bacias $\mathcal{B}\left(\Lambda_{f}\right)$ e $\mathcal{B}\left(\Lambda_{g}\right)$ são conjuntos genéricos, a saber: são interseção de subconjuntos abertos e densos do intervalo $I$. Resta saber se estas bacias tem medida de Lebesgue positiva ou não.

Existem aplicações unimodais como as aplicações de Feigenbaum-Tresser que possuem intervalos $J_{1} \supset J_{2} \supset J_{3} \ldots$ periódicos com períodos $k_{1}<k_{2}<k_{3}<\ldots$ Estas aplicações são chamadas de infinitamente renormalizáveis. Se $f$ é uma tal aplicação, o conjunto

$$
\Lambda_{f}=\bigcap_{i=0}^{\infty} \bigcup_{j=0}^{k_{i}-1} f^{j}\left(J_{i}\right),
$$

chamado solenóide, é compacto, invariante, coincide com o conjunto $\omega$-limite do extremo local $c$ e a órbita positiva de todo $x \in \Lambda_{f}$ é densa em $\Lambda_{f}$. Neste caso dizemos que $\Lambda_{f}$ é um conjunto minimal e portanto é um conjunto de Cantor. Também sabemos que se $f$ é uma aplicação infinitamente renormalizável de classe $C^{2}$ e o seu ponto crítico tem ordem finita, então a medida de Lebesgue de $\Lambda_{f}$ é nula. Porém a bacia de $\Lambda_{f}$ é um conjunto genérico com medida de Lebesgue total. Em particular, este tipo de aplicação unimodal não possui medida piac.

Observamos que as aplicações tendas são finitamente renormalizáveis, ou seja, possuem no máximo uma quantidade finita de intervalos periódicos. Enquanto que o conjunto $\omega$-limite do seu extremo local $c=1 / 2$ pode ser um conjunto de Cantor invariante e minimal. Porém a bacia deste conjunto é um conjunto magro (ou seja, união enumerável de conjuntos fechados de interior vazio) com medida de Lebesgue nula. Aplicações tendas $T_{\alpha} \operatorname{com} \alpha>1$, sempre possui uma medida piac. No entanto, resta saber se existem aplicações unimodais $f$ de classe $C^{\infty}$, com ponto crítico de ordem finita e topologicamente conjugadas à aplicações tendas, que exibem conjuntos invariantes minimais $\Lambda_{f}$ cuja bacia $\mathcal{B}\left(\Lambda_{f}\right)$ tem medida de Lebesgue positiva. Este é o conteúdo do teorema visto em [BKNvS96] que trabalharemos a seguir. Para enunciá-lo vamos considerar as aplicações unimodais da forma $g_{\gamma}(x)=\gamma-x^{2 \ell}$, para $\ell>1$ e $\gamma$ escolhido de modo que $g_{\gamma}$ possui um intervalo invariante $I=I_{\gamma}$.

Teorema B.[H. Bruin, G. Keller, T. Nowicki e S. van Strien, 1996] Se $g_{\gamma}$ é aplicação de Fibonacci e $\ell$ é suficientemente grande, o conjunto $\omega$-limite do seu ponto crítico é um conjunto de Cantor minimal de medida Lebesgue nula cuja bacia tem medida de Lebesgue total.

As aplicações $g=g_{\gamma}$ do teorema B são topologicamente conjugadas com aplicações tendas, não possuem intervalos periódicos, o intervalo $\left[g^{2}(c), g(c)\right]$ é invariante, transitivo e sua bacia é um conjunto genérico com medida de Lebesgue nula. 


\section{Capítulo 2}

\section{Ferramentas Topológicas e Combinatórias}

\subsection{Itinerários, equivalência combinatória e topológica}

Como já comentamos, o extremo local (ou ponto crítico) $c \in I$ de uma aplicação unimodal $f$ desempenha um papel fundamental para a descrição das propriedades topológicas e métricas da sua dinâmica. A ordem deste ponto crítico, a maneira pela a qual a sua órbita positiva está ordenada no intervalo e o seu grau de recorrência são de grande relevância para aspectos topológicos e métricos desta dinâmica.

Neste capítulo, vamos explorar alguns aspectos combinatórios que terão consequências de naturezas topológicas e métricas. A teoria de Milnor-Thurston [MT88] é um marco inicial para a classificação combinatória e topológica das aplicações unimodais. Começamos relembrando alguns conceitos básicos.

Definição 2.1.1 Seja $f, g: I \rightarrow I$ duas aplicaçôes unimodais com extremos $c_{f}$ e $c_{g}$, respectivamente. Dizemos que $f$ é combinatoriamente equivalente a $g$, se existe um homeomorfismo $h: I \rightarrow I$ tal que $h\left(f^{n}\left(c_{f}\right)\right)=g^{n}\left(h\left(c_{g}\right)\right)$, para todo $n \geq 0$. Dizemos que $f$ é topologicamente conjugada a $g$, se $h(f(x))=g(h(x))$, para todo $x \in I$.

Observamos que duas aplicações unimodais $f$ e $g$ são combinatoriamente equivalentes se, e somente se, as órbitas críticas positivas de $f$ e $g$, denotadas por $\operatorname{orb}^{+}\left(c_{f}\right)$ e $\operatorname{orb}^{+}\left(c_{g}\right)$, estão ordenadas da mesma forma em $\mathbb{R}$. De um ponto de vista mais grosseiro e impreciso, porém bem intuitivo, podemos pensar que duas aplicações unimodais $f$ e $g$ são combinatoriamente equivalentes se, e somente se, os gráficos de todos os iterados positivos $f^{n}$ e $g^{n}$ se "dobram do mesmo modo". Inspirados nestas ideias Milnor-Thurston [MT88] passaram a associar a cada ponto do intevalo uma sequência infinita, chamada itinerário do ponto, mais precisamente temos a seguinte definição.

Definição 2.1.2 Sejam $f: I \rightarrow I$ uma aplicação unimodal $e x \in I$. O itinerário de $x$, denotado por $\nu(x)$, é a sequência infinita

$$
\nu_{f}(x)=\left(\nu_{1}(x) \nu_{2}(x) \nu_{3}(x) \cdots\right)
$$

em que

$$
\nu_{i}(x)=\left\{\begin{array}{lll}
0 & \text { se } & f^{i}(x) \in[0, c), \\
c & \text { se } & f^{i}(x)=c \\
1 & \text { se } & f^{i}(x) \in(c, 1]
\end{array}\right.
$$

Deste modo, dada uma aplicação unimodal $f$, e colocando $S=\{0, c, 1\}$ e

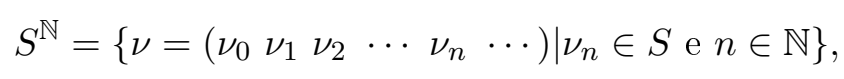


fica definida uma aplicação $\nu_{f}: I \rightarrow S^{\mathbb{N}}$, chamada aplicação itinerário de $f$, que a cada $x \in I$ associa a sequência infinita $\nu_{f}(x)$ do modo definido acima.

Como o ponto crítico é especial, o destacamos dando um nome especial para o itinerário do valor crítico $c$, a saber: $\nu_{f}(c)=\left(\nu_{1}(c) \nu_{2}(c) \nu_{3}(c) \cdots\right)$ que é chamado sequência kneading e é denotado por $k_{f}$. Então, segundo a Teoria de Milnor-Thurston [MT88], a sequência kneading determina a ordem da órbita crítica positiva em $\mathbb{R}$. Deste modo temos uma ideia da prova do teorema a seguir.

Teorema 2.1.3 Duas aplicações unimodais $f$ e g são combinatoriamente equivalentes se, e somente se, $k_{f}=k_{g}$.

A sequência kneading de uma aplicação unimodal $f$ também determina o conjunto $K_{f}$ de todas as sequências admissiveis para $f$, a saber:

$$
K_{f}=\left\{\nu=\left(\begin{array}{llll}
\nu_{1} & \nu_{2} & \nu_{3} & \ldots
\end{array}\right): \text { existe } x \in I \text { tal que } \nu(x)=\nu\right\} .
$$

Uma maneira de provar este fato é introduzindo uma ordem total no conjunto de todas as sequências infintas de símbolos no conjunto $S=\{0, c, 1\}$. Inicialmente definimos uma ordem < em $S$ pondo $0<c<1$. Então, considere duas sequências infinitas $\underline{x}=\left(\begin{array}{llllll}x_{1} & x_{2} & x_{3} & \cdots & x_{n} & \cdots\end{array}\right)$ e $\underline{y}=\left(\begin{array}{lllll}y_{1} & y_{2} & y_{3} & \cdots & y_{n}\end{array} \cdots\right)$ em $S^{\mathbb{N}}$. Dizemos que $\underline{x} \prec \underline{y}$ se, e somente se, existe $n>0$ tal que $x_{i}=y_{i}$, para todo $1 \leq i<n, x_{n} \neq y_{n} \mathrm{e}$



(b) $y_{n}<x_{n}$, se $\#\left\{i<n \mid x_{i}=1\right\}$ é ímpar.

Considerando a ordem crescente no intervalo $I$ e a ordem $\prec$ em $S^{\mathbb{N}}$, a aplicação itinerário $\nu_{f}: I \rightarrow S^{\mathbb{N}}$, preserva ordem. Mais detalhadamente, temos:

(a) se $x<y$, então $\nu_{f}(x) \preceq \nu_{f}(y)$.

(b) se $\nu_{f}(x) \prec \nu_{f}(y)$, então $x<y$.

Relembramos que se $x \neq y$ são tais que $\nu_{f}(x)=\nu_{f}(y)$, então a órbita positiva do intervalo $[x, y]$ jamais encontra o extremo local $c_{f}$. Neste caso todos os iterados $f^{n}$ são homeomorfismos quando restritos a este intervalo e ele recebe o nome de homterval. Então, pode ser que este homterval $[x, y]$ esteja contido na bacia de uma órbita periódica atratora ou pode ser que isto não ocorra e $f^{m}([x, y]) \cap f^{n}([x, y])=\emptyset$, para todo $0 \leq m<n$. Neste último caso dizemos que $[x, y]$ é um intervalo errante. Sendo assim, se $f$ não possui órbitas periódicas atratoras e também não possui intervalos errantes, a aplicação itinerário $\nu_{f}: I \rightarrow K_{f}$ é uma bijeção.

Para uma melhor compreensão da aplicação itinerário $\nu_{f}$ é necessário termos uma melhor descrição da sua imagem que é de fato o conjunto $K_{f}$ de todos os itinerários admissíveis para $f$. Mas como $f(x) \leq f(c)$, para todo $x \in I$, devemos ter que $\nu_{f}(x) \preceq k_{f}=\nu_{f}(c)$. Mais ainda, como podemos aplicar esta desigualdade em $f^{i}(x)$ ao invés de $x$, para todo $i \geq 0$, temos que $\nu_{f}\left(f^{i}(x)\right) \preceq k_{f}$. Usando a aplicação shift $\sigma: S^{\mathbb{N}} \rightarrow S^{\mathbb{N}}$, que é definida por $\sigma\left(\left(x_{1} x_{2} x_{3} \ldots\right)\right)=\left(x_{2} x_{3} \ldots\right)$, podemos ver que vale a equação $\nu_{f}(f(x))=\sigma\left(\nu_{f}(x)\right)$. Então, para que uma sequência $\underline{x} \in S^{\mathbb{N}}$ pertence a $K_{f}$ é necessário que $\sigma^{i}(x) \preceq k_{f}$. Finalmente, podemos caracterizar as sequências $\underline{x} \in K_{f}$ como sendo as sequências que satisfazem as seguintes condições:

(a) $\sigma^{i}(x) \preceq k_{f}$, para todo $i \geq 0$;

(b) se $x_{i}=c$, então $\sigma^{i}(\underline{x})=k_{f}$.

Desta forma, podemos concluir que duas aplicações unimodais $f$ e $g$ que são combinatoriamente equivalentes, e isto é equivalente a terem sequências kneading iguais, tem os mesmos conjuntos de itinerários admissíveis. 
Ainda podemos munir o conjunto $\{0, c, 1\}^{\mathbb{N}}$ de uma distância. Começamos colocando a métrica discreta $\delta$ em $S=\{0, c, 1\}$, ou seja, para $i, j \in S$ colocamos $\delta(i, i)=0$ e, se $i \neq j$, colocamos

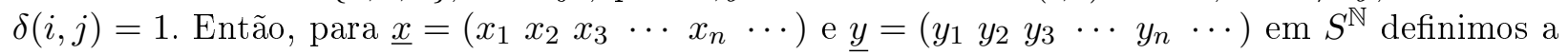
distância entre $\underline{x}$ e $\underline{y}$ pela métrica:

$$
\operatorname{dis}(\underline{x}, \underline{y})=\sum_{i=1}^{\infty} \frac{\delta\left(x_{i}, y_{i}\right)}{2^{i}}
$$

Então, considerando a distância euclidiana no intervalo $I$ e a distância em $S^{\mathbb{N}}$, a aplicação itinerário é contínua. Se $f$ não possui órbitas periódicas atratoras e também não possui intervalos errantes, a aplicação itinerário $\nu_{f}: I \rightarrow K_{f}$ é um homeomorfismo que conjuga $f$ com o shift $\sigma: K_{f} \rightarrow K_{f}$. Observe aqui que $K_{f}$ é invariante pelo shift $\sigma$. Portanto, fica delineada a prova do seguinte teorema.

Teorema 2.1.4 Se $f$ e g são duas aplicações unimodais que tem as mesmas sequências kneading, não possuem órbitas periódicas atratoras e não possuem intervalos errantes, então f e g são topologicamente conjugadas.

\section{Demonstração:}

Basta obervarmos que $h=\nu_{f} \nu_{g}^{-1}$ é uma conjugação topológica entre $f$ e $g$.

\subsection{Tempos de Corte e a Aplicação Kneading}

Aspectos métricos da dinâmica de uma aplicação unimodal $f$ de classe $C^{1}$ depende de uma competição entre uma certa expansividade que ocorre em regiões mais afastadas do ponto crítico e uma contração arbitrariamente grande em regiões próximas deste ponto. A medida que iteramos $f$ a expansividade tende a ganhar da contração, a menos que esta última se realimente muito frequentemente. Isto depende da força de recorrência do ponto crítico que pode ser medida através da sequência de tempos de primeira aproximação do ponto crítico introduzida no capítulo anterior. No entanto, não é claro quais são as sequências de inteiros positivos que podem ser realizadas como sequência de primeira aproximação para alguma aplicação unimodal $f$. Passamos então a analisar a órbita negativa do ponto crítico que também é bem natural uma vez que ela determina os pontos críticos das iteradas $f^{n}$.

Os intervalos $J_{n} \subset I$, máximos nos quais $f^{n}$ é monótona, são chamados de intervalos de monotonicidade. Observe que o bordo de um intervalo de monotonicidade $J_{n}$ são pontos da órbita negativa de $c$ ou estão no bordo de $I$. Já os pontos do bordo de $f^{n}\left(J_{n}\right)$ pertencem a órbita crítica positiva. Em particular, sempre existe $0 \leq i<n$ tal que o bordo de $f^{i}\left(J_{n}\right)$ contém o ponto crítico $c$. Sendo assim dedicamos mais atenção aos intervalos de monotonicidade cujo bordo contém $c$. Se $J_{n}$ é um tal intervalo, chamamos a restrição $f_{\mid J_{n}}^{n}$ de ramo central. Para cada $n \geq 1$, sempre existem dois ramos centrais com a mesma imagem. Se esta imagem contém $c$ dizemos que $n$ é um tempo de corte. Denotamos por $\left\{S_{k}\right\}_{k \geq 0}$ a sequência dos tempos de corte de $f$ e por $\left\{J_{S_{k}}\right\}_{k \geq 0}$, a correspondente sequência dos domínios dos ramos centrais a esquerda de $c$. Observe que se a imagem do bordo de $I$ não é um atrator periódico, então $f$ possui um ponto fixo que inverte orientação. Se este ponto também não é um atrator periódico, então $S_{0}=1 \mathrm{e} J_{s_{0}}=(a, c)$, onde $a \in \partial I$. Existe o ponto $z_{0} \in J_{s_{0}}$ tal que $f^{S_{0}}\left(z_{0}\right)=c$. Em geral, existe $z_{k} \in J_{S_{k-1}}$ tal que $f^{S_{k}}\left(z_{k}\right)=c$. A sequência $\left\{z_{k}\right\}_{k \geq 0}$ é constituída de pontos da órbita negativa de $c$ e são chamados de pontos pré-críticos mais próximos. Também consideramos a sequência $\left\{\hat{z}_{k}\right\}_{k \geq 0}$, onde $\hat{z}_{k}>c$ são tais que $f^{S_{k}}\left(z_{k}\right)=f^{S_{k}}\left(\hat{z}_{k}\right)=c$

O lema a seguir vai nos garantir posteriormente que a diferença entre dois tempos de corte consecutivos também é um tempo de corte.

Lema 2.2.1 Sejam $f$ uma aplicação unimodal, $\left\{S_{k}\right\}_{k \geq 0}$ seus tempos de corte e $\left\{z_{k}\right\}_{k \geq 0}$ seus pontos pré-críticos mais próximos. Então, $f^{S_{k-1}}\left(z_{k}\right)$ é um ponto pré-crítico mais próximo. 


\section{Demonstração:}

Seja $y:=f^{S_{k-1}}\left(z_{k}\right)$. Assim, $y \in f^{-\left(S_{k}-S_{k-1}\right)}(c)$, então temos um ponto pré-crítico. Suponhamos que $y$ não seja um ponto pré-crítico mais próximo, então existe um $z_{n} \in(y, c)$ (ou $\widehat{z}_{n} \in(c, y)$ ) tal que $S_{n}<S_{k}-S_{k-1}$. Observe na figura 2.1



Figura 2.1: Aplicação $f^{S_{k}}$ para construção da Kneading

Voltando $z_{n}$ (ou $\widehat{z}_{n}$ ) via o ramo $\left.f^{S_{k-1}}\right|_{\left(z_{k-1}, z_{k}\right)}$ temos um ponto pertecente a

$$
f^{-S_{n}-S_{k-1}}(c) \cap\left(z_{k-1}, z_{k}\right) .
$$

O que contradiz o fato de $z_{k}$ ser um ponto pré-crítico mais próximo.

Vimos que $y \in f^{-\left(S_{k}-S_{k-1}\right)}(c)$ é um ponto pré-crítico mais próximo, sendo assim $S_{k}-S_{k-1}$ é um tempo de corte. Sendo introduzida por Hofbauer e Keller em [HK90], as aplicações Kneading foram desenvolvidas com o intuito de se entender melhor as aplicações unimodais e exercerão ao longo deste trabalho um papel de grande importância. Como $S_{k}-S_{k-1}$ é um tempo de corte, podemos definir então a aplicação Kneading, $Q: \mathbb{N} \rightarrow \mathbb{N}$ por:

$$
S_{k}-S_{k-1}=S_{Q(k)} .
$$

Para conhecermos um pouco mais as aplicações Kneading, temos a seguinte proposição.

Proposição 2.2.2 Seja $f$ uma aplicação unimodal e $Q(k)$ sua aplicação Kneading. Então

$$
f^{S_{k-1}}(c) \in\left(z_{Q(k)-1}, z_{Q(k)}\right) \cup\left(\hat{z}_{Q(k)}, \hat{z}_{Q(k)-1}\right) .
$$

\section{Demonstração:}

Sabemos que $\left.f^{S_{k}}\right|_{\left[z_{k-1}, c\right]}$ e $\left.f^{S_{Q(k)}}\right|_{\left[c, \hat{z}_{Q(k)-1}\right]}$ são monótonas. Assim, $\left.f^{Q(k)}\right|_{\left[c, f^{\left.S_{k-1}(c)\right]}\right.}$ também é monótona. Observe a figura 2.2.

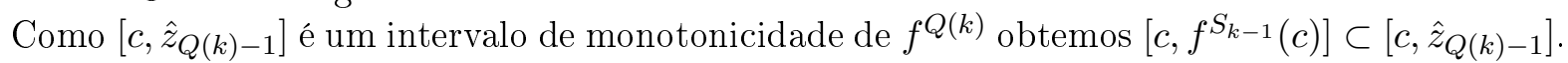
Portanto, neste caso $f^{S_{k-1}}(c) \in\left(z_{Q(k)-1}, z_{Q(k)}\right) \cup\left(\hat{z}_{Q(k)}, \hat{z}_{Q(k)-1}\right)$. 


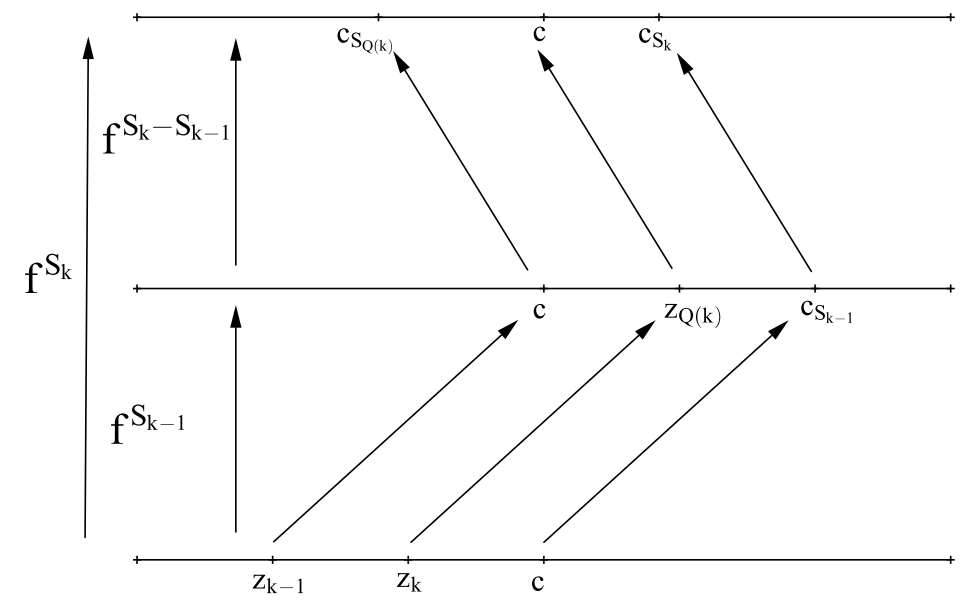

Figura 2.2: Aplicação $f^{S_{k}}$ para $f^{S_{k-1}}(c) \in\left(z_{Q(k)-1}, z_{Q(k)}\right) \cup\left(\hat{z}_{Q(k)}, \hat{z}_{Q(k)-1}\right)$

Em decorrência deste fato, temos o seguinte lema

Lema 2.2.3 Se $f$ não possui atrator periódico então $Q(k)<k$, para todo $k \geq 1$

\section{Demonstração:}

Suponha que $Q(k) \geq k$ para algum $k$. Pela proposição 2.2 .2 sabemos que

$$
f^{S_{k-1}}(c) \in\left(z_{Q(k)-1}, z_{Q(k)}\right) \cup\left(\hat{z}_{Q(k)}, \hat{z}_{Q(k)-1}\right) .
$$

Sendo assim, ficamos com

$$
f^{S_{k-1}}(c) \in\left(z_{k-1}, \hat{z}_{k-1}\right)
$$

Além disso, a aplicação $f^{S_{k-1}}$ aplica $\left(z_{k-1}, c\right)$ e $\left(c, \hat{z}_{k-1}\right)$ monotonicamente em $\left(c, c_{S_{k-1}}\right)$. Consequentemente, a aplicação $f^{S_{k-1}}$ aplica $\left(z_{k-1}, c\right)$ ou $\left(c, \hat{z}_{k-1}\right)$ nele próprio, fornecendo um ponto periódico atrator.

Seja, $H_{n-1}$ um intervalo de monotonicidade de $f^{n-1}$ contendo $c_{1}$. Por construção, quando

$$
f^{n-1}\left(H_{n-1} \cap\left[c_{2}, c_{1}\right]\right) \ni c,
$$

dizemos que $n$ um tempo de corte. Se $f^{n-1}\left(H_{n-1} \backslash\left[c_{2}, c_{1}\right]\right) \ni c$, então dizemos que $n$ é um tempo de co-corte. Denotaremos por:

$$
\left\{\tau_{k}\right\}_{k \geq 0}, \text { onde } \tau_{0}:=\min \left\{n>1 \mid c_{n} \in\left(c, c_{1}\right]\right\}
$$

Assim, perante a definição de tempo de corte e de tempo de co-corte temos

$$
S_{i} \neq \tau_{j} \text { para todo } i, j \in \mathbb{N} \text {. }
$$

Além disso, observaremos na proposição a seguir que a diferença entre tempos de co-corte consecutivos é um tempo de corte. 
Proposição 2.2.4 Seja $f$ uma aplicação unimodal e $Q(k)$ sua aplicação Kneading. Então, para cada $k \geq 1$ existe $i \in \mathbb{N}$ tal que

$$
\tau_{k}-\tau_{k-1}=S_{i} .
$$

Isto é, a diferença entre dois tempos de co-corte consecutivos é um tempo de corte.

\section{Demonstração:}

Sejam $y_{\tau_{k-1}}$ e $y_{\tau_{k}}$ pontos pré-críticos tais que

$$
f^{\tau_{k-1}}\left(y_{\tau_{k-1}}\right)=c \text { e } f^{\tau_{k}}\left(y_{\tau_{k}}\right)=c .
$$

Defina $z:=f^{\tau_{k-1}}\left(y_{\tau_{k}}\right)$. Como $f^{\tau_{k}-\tau_{k-1}}(z)=c$, logo $z \in f^{-\tau_{k}+\tau_{k-1}}(c)$. Assim, concluímos que $z$ é um ponto pré-crítico. Agora, suponha que $z$ não seja um ponto pré-crítico mais próximo. Assim, existe $z_{n} \in(z, c)$ (ou $\left.\hat{z}_{n} \in(c, z)\right)$ tal que $S_{n}<\tau_{k}-\tau_{k-1}$. Tomando a pré-imagem de $z_{n}$ pelo ramo $\left.f^{\tau_{k-1}}\right|_{\left(y_{\tau_{k}}, y_{\tau_{k-1}}\right)}$ temos $f^{-S_{n}-\tau_{k-1}} \cap\left(y_{\tau_{k}}, y_{\tau_{k-1}}\right) \neq \emptyset$. Com isso, obtemos uma contradição pois não existem pontos pré-crítico em $\left(y_{\tau_{k}}, y_{\tau_{k-1}}\right)$. Portanto, $z$ é um ponto pré-crítico mais próximo, ou seja, $\tau_{k}-\tau_{k-1}$ é tempo de corte.

\subsection{Aplicação Kneading e a sequência kneading}

Vimos que ao determinarmos a sequência de tempos de corte estamos definindo de maneira única a aplicação kneading. Além disso, ao determinarmos a aplicação kneading estamos definindo unicamente os tempos de corte da aplicação. Vale salientar que, como foi mostrado em [Bru94a], os tempos de corte de uma aplicação unimodal podem ser redefinidos a partir da sequência kneading como a seguir:

$$
S_{0}=1 \text { e } S_{k}=\min \left\{n>S_{k-1} \mid \nu_{n} \neq \nu_{n-S_{k-1}}\right\} .
$$

Assim, percebemos que ao termos uma aplicação kneading determinamos unicamente a sequência kneading. Agora, sabemos que através da sequência kneading, podemos obter os tempos de corte e consequentemente a aplicação kneading estará bem determinada.

Exemplo 2.3.1 Duas famosas aplicações unimodais podem ser descritas pela aplicação kneading

(a) Aplicação de Feigenbaum

Para esta aplicação os tempos de corte são definidos da seguinte forma: $S_{k}=2^{k}$.

Consequentemente, sua órbita crítica descreve a seguinte sequência de símbolos:

$$
\nu=10111010101110111011101010111010 \cdots
$$

Desta forma, segundo a condição de admissibilidade, temos $\nu$ admissivel.

Como $S_{Q(k)}=S_{k}-S_{k-1}=2^{k}-2^{k-1}=2^{k-1}=S_{k-1}$ obtemos para todo $k \geq 1$ que $Q(k)=k-1$.

(b) Aplicação de Fibonacci

Para esta aplicação os tempos de corte são definidos da seguinte forma: $S_{0}=1, S_{1}=2$ e $S_{k}=S_{k-1}+S_{k-2}$.

Consequentemente, sua órbita crítica descreve a seguinte sequência de símbolos:

$$
\nu=100111011001010011100 \cdots
$$

Desta forma, segundo a condição de admissibilidade, temos $\nu$ admissivel.

Como $S_{Q(k)}=S_{k}-S_{k-1}=S_{k-1}+S_{k-2}-S_{k-1}=S_{k-2}$ obtemos que $Q(k)=\max \{k-2,0\}$. 
No livro [BB04] podemos encontrar outras propriedades interessantes à respeito das aplicações Kneading e no Capítulo 5 veremos com mais detalhes as aplicações de Fibonacci. 


\section{Capítulo 3}

\section{Ferramentas Métricas}

Neste capítulo, reuniremos diversos conceitos básicos de natureza métrica que terão importância no desenvolvimento dos capítulos seguintes. Tendo como objetivo principal contruir as medidas invariantes através das aplicações induzidas, abordaremos propriedades métricas que nos ajudarão a obter boas propriedades de distorção.

\subsection{Derivada Schwarziana}

O conceito que vamos estudar nesta seção foi utilizada em 1978 por D. Singer em dinâmica unidimensional [Sin78]. Ela é chamada de derivada de Schwarz e tem um papel relevante neste trabalho e de maneira geral tem grande importância no estudo de dinâmica de funções complexas.

Definição 3.1.1 Seja $f: I \rightarrow I$ uma aplicação unimodal de classe $C^{3}$. Assim, a derivada de Schwarz da função $f$ é dada por

$$
\mathcal{S} f=\frac{D^{3} f}{D f}-\frac{3}{2}\left(\frac{D^{2} f}{D f}\right)^{2}
$$

no complementar do ponto crítico de $f$. Dizemos que $f$ tem derivada Schwarziana negativa se $\mathcal{S} f<0$ no complementar do ponto crítico de $f$.

Vejamos dois exemplos de funções com derivada Schwarziana negativa

Exemplo 3.1.2 Seja $f_{a}:[0,1] \rightarrow[0,1]$ definida por $f_{a}(x)=a \sin (\pi x)$ com $a \in(0,1]$. Como

- $D f(x)=a \pi \cos (\pi x)$,

- $D^{2} f(x)=-a \pi^{2} \sin (\pi x)$,

- $D^{3} f(x)=-a \pi^{3} \cos (\pi x)$.

Assim,

$$
\mathcal{S} f(x)=-\pi^{2}-\frac{3}{2}\left(\frac{-\pi \sin (\pi x)}{\cos (\pi x)}\right)^{2}=-\pi^{2}\left(1+\frac{3}{2} \tan ^{2}(\pi x)\right)<0
$$

$\operatorname{para} x \neq \frac{1}{2}$.

Exemplo 3.1.3 Seja $g_{\beta}:[0,1] \rightarrow[0,1]$ definida por $g_{\beta}(x)=\beta x(1-x)$ com $\beta \in(0,4]$. Como

- $D g_{\beta}(x)=\beta-2 \beta x$,

- $D^{2} g_{\beta}(x)=-2 \beta$,

- $D^{3} g_{\beta}(x)=0$. 
Assim,

$$
\mathcal{S} g_{\beta}(x)=-\frac{3}{2}\left(\frac{-2 \beta}{\beta-2 x \beta}\right)^{2}=\frac{-6}{(1-2 x)^{2}}<0
$$

para $x \neq \frac{1}{2}$.

Propriedade 3.1.4 Seja $f$ uma função real de classe $C^{3}$ e sem pontos críticos. A derivada Schwarziana de $f$ é identicamente nula se, e somente se, $f$ é uma transformação de Moebius.

\section{Demonstração:}

Se $f$ é uma transformação de Moebius então $f(x)=\frac{a+b x}{c+d x}$ tal que $a d-b c \neq 0$. Assim, devemos calcular $D f, D^{2} f$ e $D^{3} f$ :

- $D f(x)=\frac{b c-a d}{(c+d x)^{2}}$

- $D^{2} f(x)=\frac{-2 d(b c-a d)}{(c+d x)^{3}}$

- $D^{3} f(x)=\frac{6 d^{2}(b c-a d)}{(c+d x)^{4}}$

Logo,

$$
\mathcal{S} f(x)=\frac{\frac{6 d^{2}(b c-a d)}{(c+d x)^{4}}}{\frac{b c-a d}{(c+d x)^{2}}}-\frac{3}{2}\left(\frac{\frac{-2 d(b c-a d)}{(c+d x)^{3}}}{\frac{b c-a d}{(c+d x)^{2}}}\right)^{2}=\frac{6 d^{2}}{(c+d x)^{2}}-\frac{6 d^{2}}{(c+d x)^{2}}=0
$$

Por outro lado, suponhamos $\mathcal{S} f \equiv 0$.

Considere a função $g=\frac{f^{\prime \prime}}{f^{\prime}}$. Assim, $g$ satisfaz a equação diferencial

$$
g^{\prime}=\frac{g^{2}}{2}
$$

cuja solução é dada por $g \equiv 0$ ou $g(x)=\frac{-2}{x+p}$, sendo $p$ uma constante real.

Logo, se $\frac{f^{\prime \prime}}{f^{\prime}}=0$ obtemos $f(x)=a x+b$ e se $\frac{f^{\prime \prime}}{f^{\prime}}=\frac{-2}{x+p}$ obtemos $f(x)=\frac{a x+b}{c x+d}$, sendo $a, b, c$ e $d$ constantes reais.

Uma das principais razões de utilizarmos a derivada de Schwarz é que ela se adapta ao estudo de sistemas dinâmicos por ter um bom comportamento perante à composição de funções.

Proposição 3.1.5 Sejam g e $\phi$ funções reais de classe $C^{3}$. Então

$$
\mathcal{S}(g \circ \phi)=(\mathcal{S} g \circ \phi) \cdot\left(\phi^{\prime}\right)^{2}+\mathcal{S} \phi .
$$

\section{Demonstração:}

Vamos calcular as seguintes derivadas apenas aplicando a regra da cadeia

$$
\begin{gathered}
(g \circ \phi)^{\prime}=g^{\prime}(\phi) \phi^{\prime} \\
(g \circ \phi)^{\prime \prime}=g^{\prime \prime}(\phi)\left(\phi^{\prime}\right)^{2}+g^{\prime}(\phi) \phi^{\prime \prime} \\
(g \circ \phi)^{\prime \prime \prime}=g^{\prime \prime \prime}(\phi)\left(\phi^{\prime}\right)^{3}+3 g^{\prime \prime}(\phi) \phi^{\prime} \phi^{\prime \prime}+g^{\prime}(\phi) \phi^{\prime \prime \prime}
\end{gathered}
$$

Sendo assim,

$$
S(g \circ \phi)=\frac{g^{\prime \prime \prime}(\phi)\left(\phi^{\prime}\right)^{2}}{g^{\prime}(\phi)}+\frac{3 g^{\prime \prime}(\phi) \phi^{\prime \prime}}{g^{\prime}(\phi)}+\frac{\phi^{\prime \prime \prime}}{\phi^{\prime}}-\frac{3}{2}\left[\frac{g^{\prime \prime}(\phi)}{g^{\prime}(\phi)} \phi^{\prime}+\frac{\phi^{\prime \prime}}{\phi^{\prime}}\right]^{2} .
$$


Portanto,

$$
S(g \circ \phi)=\frac{g^{\prime \prime \prime}(\phi)\left(\phi^{\prime}\right)^{2}}{g^{\prime}(\phi)}-\frac{3}{2}\left(\frac{g^{\prime \prime}(\phi) \phi^{\prime}}{g^{\prime}(\phi)}\right)^{2}+\frac{\phi^{\prime \prime \prime}}{\phi^{\prime}}-\frac{3}{2}\left(\frac{\phi^{\prime \prime}}{\phi^{\prime}}\right)^{2}
$$

ou seja,

$$
\mathcal{S}(g \circ \phi)=(\mathcal{S} g \circ \phi) \cdot\left(\phi^{\prime}\right)^{2}+\mathcal{S} \phi
$$

Observamos ainda que se fizermos a composição de uma aplicação $g$ de classe $C^{3}$, que possui derivada Scharziana negativa, com uma transformação de Moebius ou com uma aplicação com derivada Schwarzina negativa, digamos $\phi$, ainda sim, teremos

$$
\mathcal{S}(g \circ \phi)(x)<0
$$

A condição da derivada de Schwarz negativa tem propriedades boas em relação a composição de funções, pois se $g$ e $\phi$ a satisfazem, $g \circ \phi$ também a satisfaz. Temos então, uma propriedade que se propaga quando olhamos para seus iterados.

\subsection{Distorção e a razão cruzada}

Nesta seção estudaremos ferramentas que serão bastante utilizadas, principalmente no Capítulo 5: A razão cruzada e o controle da distorção. Além disso, veremos a derivada Schwarziana realizar um papel importante neste contexto.

A distorção é a medida da não-linearidade da função e pode ser definida como o supremo da razão dos valores absolutos da derivada em dois pontos distintos, isto é:

Definição 3.2.1 Seja $g: J \rightarrow g(J)$ uma aplicação monótona $C^{1}$. O valor

$$
\operatorname{dis}(g, J):=\sup _{x, y \in J} \frac{|D g(x)|}{|D g(y)|}
$$

é chamado de distorção de g em J. Sendo J um intervalo limitado da reta.

Denotaremos por $(J, T)$ um par de intervalos abertos e limitados da reta tal que $\bar{J} \subset T$, e por $L$ e $R$ as componentes conexas de $T \backslash J$.

Definição 3.2.2 Para cada par de intervalos $(J, T)$ defina a razão cruzada dos intervalos $T$ e $J$ por

$$
D(T, J)=\frac{|J||T|}{|L||R|}
$$

onde $|I|$ denota o comprimento do intervalo $I$.

Definição 3.2.3 Se $g: I \rightarrow \mathbb{R}$ é contínua e monótona definimos a distorção da razão cruzada como

$$
B(g, T, J)=\frac{D(g(T), g(J))}{D(T, J)},
$$

para cada para de intervalos $(J, T)$ contidos em $I$.

Sendo assim, vejamos duas proposições importantes que se inserem neste contexto.

Proposição 3.2.4 Seja $g: I \rightarrow \mathbb{R}$ uma função contínua e monótona. Então, para qualquer par de intervalos $(J, T)$ contidos em I tem-se que

$$
B(g, T, J)=1
$$


se, e somente se, g é a restrição de uma tranformação de Moebius em T.

\section{Demonstração:}

Seja $T=\left[x_{1}, x_{2}\right]$. Suponhamos inicialmente que $g$ é uma transformação de Moebius. Assim, podemos escrever $g$ como sendo

$$
g(x)=\frac{a x+b}{c x+d}=\frac{a}{c}-\frac{a d-b c}{c} \cdot \frac{1}{c x+d},
$$

para $c \neq 0$ e $x \in \mathbb{R}$. Seja $J=\left[x_{3}, x_{4}\right]$. Como $g$ é uma função monótona, obtemos

$$
\begin{aligned}
D(g(T), g(J)) & =\frac{|g(J)||g(T)|}{|g(L)||g(R)|} \\
& =\frac{\left|g\left(x_{3}\right)-g\left(x_{4}\right)\right|\left|g\left(x_{1}\right)-g\left(x_{2}\right)\right|}{\left|g\left(x_{1}\right)-g\left(x_{3}\right)\right|\left|g\left(x_{4}\right)-g\left(x_{2}\right)\right|} \\
& =\frac{\left|x_{3}-x_{4}\right|\left|x_{1}-x_{2}\right|}{\left|x_{1}-x_{3}\right|\left|x_{4}-x_{2}\right|} \\
& =\frac{|J||T|}{|L||R|} \\
& =D(T, J) .
\end{aligned}
$$

Portanto, $B(g, T, J)=1$. Por outro lado, suponhamos $B(g, T, J)=1$ e fixemos um ponto $x_{0} \in\left(x_{1}, x_{2}\right)$. Já que $x_{1}, x_{2}$ e $x_{0}$ são distintos, existe uma única transformação de Moebius $\phi$ que satisfaz

$$
\begin{aligned}
& \phi\left(x_{0}\right)=g\left(x_{0}\right) \\
& \phi\left(x_{1}\right)=g\left(x_{1}\right) \\
& \phi\left(x_{2}\right)=g\left(x_{2}\right) .
\end{aligned}
$$

Mostraremos que $\phi(x)=g(x)$, para todo $x \in T$. De fato, tomemos $x \in\left(x_{1}, x_{2}\right)$ de maneira que $x \neq x_{0}$, suponhamos sem perda de generalidade que $x_{0}<x$, e consideremos o intervalo $J=\left(x_{0}, x\right)$. Por hipótese e pelo que provamos anteriormente, temos

$$
B(g, T, J)=B(\phi, T, J)=1 .
$$

Assim, para todo par de intervalos $(J, T)$ contidos em $I$ temos

$$
\frac{|g(J)|}{|g(R)|}=\frac{|\phi(J)|}{|\phi(R)|} .
$$

Já que $\phi(T)=g(T)$ e $\phi\left(x_{0}\right)=g\left(x_{0}\right)$ obtemos

$$
|g(J)|+|g(R)|=|\phi(J)|+|\phi(R)| .
$$

Com isso, concluímos que $|g(J)|=|\phi(J)|$ e $|g(R)|=|\phi(R)|$. Como $x$ é arbitrário, segue que $\phi(x)=f(x)$, para todo $x \in T$.

Proposição 3.2.5 Se $g: T \rightarrow \mathbb{R}$ é uma aplicação de classe $C^{3}$ com derivada Schwarziana negativa então

$$
B\left(g, T^{*}, J^{*}\right)>1
$$

Para todo $J^{*} \subset T^{*} \subset T$. 


\section{Demonstração:}

Sejam $T^{*}=\left[x_{0}, x_{1}\right]$ e $J^{*}=\left[y_{0}, y_{1}\right]$. Seja $\phi$ uma transformação de Moebius tal que a composição $\phi \circ g$ tenha como ponto fixo os pontos $x_{0}, y_{0}$ e $x_{1}$.

Afirmação: $\phi\left(g\left(y_{1}\right)\right)>y_{1}$.

Suponha que $\phi\left(g\left(y_{1}\right)\right) \leq y_{1}$. Sendo assim, pelo Teorema do Valor Médio existem $z_{0} \in\left[x_{0}, y_{0}\right]$, $z_{1} \in\left[y_{0}, y_{1}\right]$ e $z_{2} \in\left[y_{1}, x_{1}\right]$ tais que

$$
\begin{gathered}
D(\phi \circ g)\left(z_{0}\right)=\frac{\phi\left(g\left(y_{0}\right)\right)-\phi\left(g\left(x_{0}\right)\right)}{y_{0}-x_{0}}=\frac{y_{0}-x_{0}}{y_{0}-x_{0}}=1 \\
D(\phi \circ g)\left(z_{1}\right)=\frac{\phi\left(g\left(y_{1}\right)\right)-\phi\left(g\left(y_{0}\right)\right)}{y_{1}-y_{0}}=\frac{\phi\left(g\left(y_{1}\right)\right)-y_{0}}{y_{1}-y_{0}} \leq 1 \\
D(\phi \circ g)\left(z_{2}\right)=\frac{\phi\left(g\left(x_{1}\right)\right)-\phi\left(g\left(y_{1}\right)\right)}{x_{1}-y_{1}}=\frac{x_{1}-\phi\left(g\left(y_{1}\right)\right)}{x_{1}-y_{1}} \geq 1 .
\end{gathered}
$$

Como $\mathcal{S}(\phi \circ g)=\mathcal{S} g<0$ isto contradiz o Princípio do Mínimo (veremos a seguir em 3.3.1). Com isso, provamos a afirmação, ou seja, $\phi\left(g\left(y_{1}\right)\right)>y_{1}$.

Assim, temos $\left|\phi\left(g\left(J^{*}\right)\right)\right|>\left|J^{*}\right|$ e que $\left|R^{*}\right|>\left|\phi\left(g\left(R^{*}\right)\right)\right|$. Então

$$
B\left(\phi \circ g, T^{*}, J^{*}\right)=\frac{\frac{\left|\phi\left(g\left(T^{*}\right)\right)\right|}{\left|T^{*}\right|} \frac{\left|\phi\left(g\left(J^{*}\right)\right)\right|}{\left|J^{*}\right|}}{\frac{\left|\phi\left(g\left(L^{*}\right)\right)\right|}{\left|L^{*}\right|} \frac{\left|\phi\left(g\left(R^{*}\right)\right)\right|}{\left|R^{*}\right|}}=\frac{\left|\phi\left(g\left(J^{*}\right)\right)\right|}{\left|J^{*}\right|} \frac{\left|R^{*}\right|}{\left|\phi\left(g\left(R^{*}\right)\right)\right|}>1
$$

Como $B\left(\phi \circ g, T^{*}, J^{*}\right)=B\left(\phi, g\left(T^{*}\right), g\left(J^{*}\right)\right) \cdot B\left(g, T^{*}, J^{*}\right)$, e como a transformação de Moebius preserva a razão cruzada, temos $B\left(g, T^{*}, J^{*}\right)>1$.

Assim, como trabalharemos sempre com funções unimodais $C^{3}$ com derivada Schwarziana negativa estaremos pensando em funções com a distorção da razão cruzada maior que 1.

\subsection{Princípio de Koebe}

Nesta seção temos como objetivo principal apresentar o princípio de Koebe. Contudo, é preciso conhecer inicialmente uma ferramante bastante útil: O Princípio do mínimo.

Teorema 3.3.1 (Princípio do mínimo) Seja $T=[a, b] \subset I$ e $g: T \rightarrow g(T) \subset I$ um difeomorfismo de classe $C^{1}$. Seja $x \in(a, b)$. Se para qualquer $J^{*} \subset T^{*} \subset T$

$$
B\left(g, T^{*}, J^{*}\right) \geq C_{0}>0
$$

entâo $|D g(x)| \geq C_{0}^{3} \cdot \min \{|D g(a)|,|D g(b)|\}$.

\section{Demonstração:}

Podemos tomar $T^{*}=[a, b]$ em $T$ e considere

$$
B_{0}\left(g, T^{*}\right)=\left(\frac{\left|g\left(T^{*}\right)\right|}{\left|T^{*}\right|}\right)^{2} \frac{1}{|D g(a)||D g(b)|}
$$

Além disso, defina

$$
B_{1}(g, T, x)=\frac{|D g(x)| \frac{|g(T)|}{|T|}}{\frac{|g(L)|}{|L|} \frac{|g(R)|}{|R|}}
$$

Aqui, L e R são componentes de $T \backslash\{x\}$. Observe que

$$
B_{0}\left(g, T^{*}\right)=\lim _{J^{*} \rightarrow T^{*}} B\left(g, T^{*}, J^{*}\right)
$$


$\mathrm{e}$

$$
B_{1}(g, T, x)=\lim _{T \rightarrow x} B(g, T, J) .
$$

Com isso, é fácil ver que $B_{0}(g, L), B_{0}(g, R), B_{1}(g, T, x) \geq C_{0}>0$. Obtemos então

$$
B_{0}(g, L)=\left(\frac{|g(L)|}{|L|}\right)^{2} \frac{1}{|D g(a)||D g(x)|} \geq C_{0}
$$

ou seja, $\left(\frac{|g(L)|}{|L|}\right)^{2} \geq C_{0}|D g(a)||D g(x)|$.

Além disso, temos

$$
B_{0}(g, R)=\left(\frac{|g(R)|}{|R|}\right)^{2} \frac{1}{|D g(x)||D g(b)|} \geq C_{0}
$$

que nos fornece $\left(\frac{|g(R)|}{|R|}\right)^{2} \geq C_{0}|D g(x)||D g(b)| \mathrm{e}$

$$
B_{1}(g, T, x)=\frac{|D g(x)| \frac{|g(T)|}{|T|}}{\frac{|g(L)|}{|L|} \frac{|g(R)|}{|R|}} \geq C_{0}
$$

que obtemos $|D g(x)| \frac{|g(T)|}{|T|} \geq C_{0} \frac{|g(L)|}{|L|} \frac{|g(R)|}{|R|}$.

Como $\left.g\right|_{T}$ é difeomorfismo, temos

$$
\min \left\{\frac{|g(L)|}{|L|}, \frac{|g(R)|}{|R|}\right\} \leq \frac{|g(T)|}{|T|} \leq \max \left\{\frac{|g(L)|}{|L|}, \frac{|g(R)|}{|R|}\right\}
$$

Com isso,

$$
\begin{aligned}
|D g(x)|^{2} & \geq C_{0}^{2}\left(\frac{\frac{|g(L)|}{|L|} \frac{|g(R)|}{|R|}}{\frac{|g(T)|}{|T|}}\right)^{2} \\
& \geq C_{0}^{2} \min \left\{\left(\frac{|g(L)|}{|L|}\right)^{2},\left(\frac{|g(R)|}{|R|}\right)^{2}\right\} \\
& \geq C_{0}^{3} \min \{|D g(a)||D g(x)|,|D g(b)||D g(x)|\}
\end{aligned}
$$

Portanto, $|D g(x)| \geq C_{0}^{3} \min \{|D g(a)|,|D g(b)|\}$

A partir deste resultado, podemos discutir uma das ferramentas importantes da dinâmica unidimensional: O princípio de Koebe. O princípio de Koebe juntamente com a condição da função unimodal ter derivada Schwarziana negativa nos fornece um certo controle para não-linearidade das iteradas da função, o que nos permite estudar certas propriedades métricas, como a medida de Lebesgue de conjuntos invariantes.

Lema 3.3.2 (Princípio de Koebe) Seja $C_{0} \in(0,1]$ e suponha que $g: T \rightarrow g(T)$ é um difeomorfismo de classe $C^{1}$ com $B(g, T, J) \geq C_{0}>0$ em que $J \subset T$ e que ambas as componentes de $g(T \backslash J)$ tem tamanho maior ou igual a $\delta|g(J)|$. Então, existe $K=K\left(C_{0}, \delta\right)$ tal que

$$
\frac{1}{K\left(C_{0}, \delta\right)} \leq \frac{D g(x)}{D g(y)} \leq K\left(C_{0}, \delta\right), \quad \forall x, y \in J
$$

\section{Demonstração:}


A fim de simplicar as contas, podemos reescalar $\mathrm{J}$ de forma que $J=g(J)=[0,1]$ e $g$ crescente. Usaremos $B_{0}$, definido anteriormente no teorema do princípio do mínimo, no intervalo $J$. Assim,

$$
|D g(0)||D g(1)| \geq \frac{1}{C_{0}}\left(\frac{|g(J)|}{|J|}\right)^{2}=\frac{1}{C_{0}}
$$

Usaremos agora $B_{1}$, também definido anteriormente no teorema, em $[a, 1]$, para $x=0$. Com isso,

$$
|D g(0)| \geq C_{0} \frac{\frac{|g(L)|}{|L|} \frac{|g(J)|}{|J|}}{\frac{|g(L \cup J)|}{|L \cup J|}}
$$

Como $|g(J)|=|J|=1,|g(L)| \geq \delta|g(J)|$ e como $|g(L \cup J)|=|g(L)|+|g(J)|$. Temos

$$
\begin{aligned}
\frac{|g(L)|}{|L|} \frac{|L \cup J|}{|g(L \cup J)|} & =\frac{|g(L)|}{|g(L \cup J)|} \frac{|L \cup J|}{|L|} \\
& \geq \frac{|g(L)|}{|g(L \cup J)|} \\
& =\frac{|g(L)|}{|g(L)|+|g(J)|} \\
& \geq \frac{|g(L)|}{|g(L)|+\frac{|g(L)|}{\delta}} \\
& =\frac{\delta}{1+\delta}
\end{aligned}
$$

Logo, voltando para (3.4), ficamos com

$$
|D g(0)| \geq C_{0} \frac{\delta}{1+\delta}
$$

Usando os mesmo argumentos para o intervalo $[0, b]$ para $x=1$ concluimos que

$$
|D g(1)| \geq C_{0} \frac{\delta}{1+\delta}
$$

Com isso, observando (3.3) obtemos

$$
C_{0} \frac{\delta}{1+\delta} \leq|D g(0)|,|D g(1)| \leq \frac{1}{C_{0}^{2}} \frac{1+\delta}{\delta}
$$

Pelo princípio do mínimo, resultamos em

$$
|D g(x)| \geq C_{0}^{4} \frac{\delta}{1+\delta} \quad \forall x \in[0,1] .
$$

Assim, qualquer que seja $x \in(0,1)$ defina $L_{x}=[0, x]$ e $R_{x}=[x, 1]$. Como $g$ é um difeomorfismo ocorrerá apenas um dos casos

- $\frac{\left|g\left(L_{x}\right)\right|}{\left|L_{x}\right|} \leq \frac{|g(J)|}{|J|}=1 ;$

- $\frac{\left|g\left(R_{x}\right)\right|}{\left|R_{x}\right|} \leq \frac{|g(J)|}{|J|}=1$. 
Suponha sem perda de generalidade que $\frac{\left|g\left(L_{x}\right)\right|}{\left|L_{x}\right|} \leq 1$. Assim usando (3.3) no intervalo $L_{x}$ temos

$$
\frac{\left(\frac{\left|g\left(L_{x}\right)\right|}{\left|L_{x}\right|}\right)^{2}}{|D g(0)||D g(x)|} \geq C_{0}
$$

Com isso, $|D g(0)||D g(x)| \leq \frac{1}{C_{0}}$. Assim, por (3.5) e por (3.6) concluimos que para todo $x \in J$ temos

$$
C_{0}^{4} \frac{\delta}{1+\delta} \leq|D g(x)| \leq \frac{1}{C_{0}^{2}} \frac{1+\delta}{\delta} .
$$

Logo,

$$
C_{0}^{6}\left(\frac{\delta}{1+\delta}\right)^{2} \leq \frac{D g(x)}{D g(y)} \leq \frac{1}{C_{0}^{6}}\left(\frac{1+\delta}{\delta}\right)^{2}:=K\left(C_{0}, \delta\right)
$$

O Princípio de Koebe provado acima continua válido no caso em que $\mathcal{S} g<0$, pois anteriormente precisávamos supor que $B(g, T, J) \geq C_{0}>0$ mas vimos pela propriedade 3.2 .5 que se $\mathcal{S} g<0$ então $B\left(g, T^{*}, J^{*}\right)>1$. Logo, a constante de distorção, $\mathrm{K}$, vai depender apenas do $\delta$. Assim,

$$
\operatorname{dis}(g, J)=\sup _{x, y \in J} \frac{|D g(x)|}{|D g(y)|} \leq K(\delta)
$$

As componentes de $g(T \backslash J)$ são chamadas de espaços de Koebe.

\subsection{Aplicações de Markov}

Uma aplicação importante e muito utilizada neste trabalho são as aplicações de Markov. Por exemplo, para construirmos as medidas invariantes no Capítulo 4 utilizaremos as aplicações induzidas de Markov.

Definição 3.4.1 (Aplicações de Markov) Uma aplicação $F:[0,1] \rightarrow[0,1]$ é chamada de aplicação de Markov se existe uma família enumerável de intervalos abertos disjuntos $Y_{i}$ em $[0,1]$ tais que

(i) Se $F\left(Y_{i}\right) \bigcap Y_{j} \neq \emptyset$ então $F\left(Y_{i}\right) \supset Y_{j}$. Em outras palavras, $F$ preserva a partição;

(ii) $\left.F\right|_{Y_{i}}$ é monótona para cada $i$.

Definição 3.4.2 Dizemos que uma aplicação $f: I \rightarrow I$ induz uma aplicação de Markov se existe um intervalo $J \subset I$ e uma aplicação de Markov F em $J$, definido num subconjunto $\bigcup_{j=1}^{\infty} I_{j}$ de $J$ com medida de Lebesgue total tal que, cada $j \in \mathbb{N}$, a restrição de $F$ em $I_{j}$ é um iterado $f^{k(j)}$ de $f$ com $f^{k(j)}\left(I_{j}\right) \subset J$. Dizemos que $F$ é induzida por $f$ em $J$.

Sabemos que a aplicação de Markov admitirá piac quando satisfizer as hipóteses do teorema de folclore. Este nome foi atribuido ao teorema pois os avanços para sua prova podem ser encontrados nos trabalhos de diversos matemáticos e, portanto, sua demonstração não pode ser atribuida a nenhum autor em específico. Uma versão deste teorema pode ser encontrada em [dMvS93].

Teorema 3.4.3 (Folclore) Seja $F$ uma aplicação de Markov no intervalo. Se existir $\beta>0 e$ $K \geq 1$ tal que dis $\left(F^{n}, J\right) \leq K$ e $\left|F^{n}(J)\right| \geq \beta$ para todo $n$ e todo ramo $\left.F^{n}\right|_{J}$, então $F$ admite piac, $m$, e a derivada de Radon-Nikodým $\mathrm{dm} / \mathrm{dx}$ é limitada. 
Contudo, quando temos uma aplicação induzida de Markov $F$ possuindo piac, em geral não implica que $f$ também tenha tal medida. Porém, vemos o seguinte

Lema 3.4.4 Seja $f: I \rightarrow I$ a aplicação que induz a aplicação de Markov $F: \bigcup_{j=1}^{\infty} I_{j} \rightarrow J$ seja $k(j)$ de modo que $\left.F\right|_{I_{j}}=f^{k(j)}$ e seja $\mu$ uma medida de probabilidade invariante absolutamente continua de F. Temos

$$
\sum_{j=1}^{\infty} k(j)\left|I_{j}\right|<\infty
$$

se, e somente se, $f$ tem medida de probabilidade invariante absolutamente continua.

\section{Demonstração:}

Ver [dMvS93].

\subsection{Resultados da Teoria da medida e ergódica}

A propriedade métrica que estaremos interessados em investigar são as medidas de probabilidade invariantes e absolutamente contínuas com respeito à medida de Lebesgue pois uma parte importante na compreensão de um sistema dinâmico consiste na compreensão das suas medidas invariantes e suas propriedades.

Definição 3.5.1 Seja $\mu$ uma medida definida na $\sigma$-álgebra de borel do espaço X. Dizemos que $\mu$ é invariante pela transformação $f$ se

$$
\mu(A)=\mu\left(f^{-1}(A)\right)
$$

para todo conjunto mensurável $A \subset X$.

Além disso, dizemos que uma medida é $\sigma$-finita se existir uma sequência de subconjuntos $V_{1}, V_{2}, \cdots$ de $X$ satisfazendo $\mu\left(V_{i}\right)<\infty$ para todo $i \in \mathbb{N}$ e

$$
X=\bigcup_{i=1}^{\infty} V_{i} .
$$

Definição 3.5.2 Sejam $\mu$ e $\nu$ duas medidas num espaço mensurável $(X, \mathcal{B})$. Dizemos que $\nu$ é absolutamente contínua em relação a $\mu$, se todo conjunto mensurável $A$ que satisfaz $\mu(A)=0$ também satisfaz $\nu(A)=0$. Neste caso, escrevemos $\nu \ll \mu$.

Exemplo 3.5.3 Seja a aplicação quadrática $f:[-1,1] \rightarrow[-1,1]$ definida por $f(x)=1-2 x^{2}$. Em dinâmica unidimensional este é um dos mais simples exemplos de aplicaçẫo que possui piac. De fato, consideremos a tenda $T:[-1,1] \rightarrow[-1,1]$ definido por $T(x)=1-2|x|$. A aplicação $h:[-1,1] \rightarrow[-1,1]$ definido por $h(x)=\frac{2}{\pi} \sin ^{-1}(x)$ é uma conjugação entre $f$ e T. Neste caso, as aplicações $T$ e $f$ admitem piac.

Um resultado importante com este enfoque, conhecido como teorema de Radón-Nikodym, afirma que quando temos $\nu \ll \mu$, a medida $\nu$ pode ser vista como a integral com respeito a $\mu$ de uma certa função mensurável $\rho$, que é chamada de densidade ou derivada de Radón-Nikodym de $\nu$ relativamente a $\mu$.

Teorema 3.5.4 (Radón-Nikodym) Se $\mu$ e $\nu$ são duas medidas finitas tais que $\nu \ll \mu$ então existe uma função mensurável $\rho: X \rightarrow[0,+\infty]$ tal que $\nu=\rho \mu$, ou seja, tal que

$$
\nu(A)=\int_{A} \rho d \mu \text { para todo conjunto mensurável } A \subset X
$$


Além disso, essa função é essencialmente única: duas quaisquer funções que as satisfazem são iguais para $\mu$-quase todo ponto.

Queremos controlar o comportamento de órbitas de maneira assintótica e a teoria ergódica oferece uma ferramenta para lidar com este problema, dado que o sistema possua alguma medida invariante suficientemente boa (por exemplo, absolutamente contínua com respeito à medida de Lebesgue). Como podemos ver pelo Teorema Ergódico de Birkhoff.

Teorema 3.5.5 (Ergódico de Birkoff) Sejam $f: X \rightarrow X$ uma transformação mensurável, $(X, \mathcal{A}, \mu)$ um espaço de probabilidade e $\mu$ uma probabilidade invariante por $f$. Dado qualquer conjunto mensurável $E \subset X$, o tempo médio visita

$$
\tau_{E}(x)=\lim _{n} \frac{1}{n} \#\left\{j=0,1, \cdots, n-1 \mid f^{j}(x) \in E\right\}
$$

existe em $\mu$ q.t.p. $x \in X$.

Definição 3.5.6 Um intervalo $J, c \in J \subset I$, é chamado de restritivo se $f^{n}(J) \subset J$ para algum $n>1$. Se um intervalo restritivo existir, $f$ é chamado de renormalizável.

Teorema 3.5.7 Seja $f$ não renormalizável e sem pontos periódicos atratores então vale a seguinte propriedade:

$$
\text { Se }|A|>0, \text { logo } \bigcup_{i} f^{-i}(A) \text { tem medida de Lebesgue total. }
$$

Aqui, $|\cdot|$ denota a medida de Lebesgue. Se $f$ é finitamente renormalizável, então 3.5.7 continuará válida se $A$ for tomado no menor intervalo restritivo de $f$. 


\section{Capítulo 4}

\section{Conjugações topológicas e a propriedade métrica}

Nosso objetivo principal neste capítulo é provar o seguinte resultado:

Teorema A. Existem aplicações unimodais $q_{\alpha}$ que possuem uma medida piac e são topologicamente conjugadas a aplicaçôes unimodais $f_{\beta}$ que não possuem medida piac.

Para provarmos este teorema utilizaremos algumas ferramentas vistas no Capítulo 2 como as aplicações Kneading e as sequências Kneading, pois estudaremos tais aplicações sob o ponto de vista combinatorial. Além disso, vamos definir as aplicações ramo-longo. Trabalhar com aplicações do tipo ramo-longo nos garantirá boas propriedades de distorção e também uma limitação das aplicações Kneading para assim contruirmos a medida invariante. Além disso, veremos que será necessário construir aplicações em que o ramo central é quase tangente a diagonal e que a derivada no ponto fixo assuma valores diferentes em cada uma das aplicações a fim de construirmos a prova do resultado.

As aplicações unimodais em questão serão aplicações de classe $C^{3}$, com derivada Schwarziana negativa, ordem crítica $\ell$, em que $1<\ell<\infty$, simétrica e sem atrator periódico. A família das aplicações que satisfazem tais condições chamaremos de $\mathcal{F}$

\subsection{Construindo a medida}

Com o objetivo de construirmos a medida vamos desvencilhar do ponto crítico ao construirmos as aplicações induzidas. Sendo assim, defina o anel

$$
A_{k}:=\left(z_{k-1}, z_{k}\right) \cup\left(\widehat{z}_{k}, \widehat{z}_{k-1}\right)
$$

Definamos também a aplicação induzida $F$ como

$$
F: \bigcup_{k \geq 1} A_{k} \rightarrow\left(z_{0}, \hat{z}_{0}\right),\left.\quad F\right|_{A_{k}}=f^{S_{k-1}}
$$

Temos então $F\left(A_{k}\right)=\left(z_{Q(k)}, c\right)$ ou $F\left(A_{k}\right)=\left(c, \hat{z}_{Q(k)}\right)$.

Exemplo 4.1.1 Vejamos exemplos desta aplicação induzida para as aplicaçôes de Fibonacci e para uma aplicação tenda.

(i) Aplicação de Fibonacci

Como vimos em 2.3.1 a sequência Kneading da aplicação de Fibonacci é dada por:

$$
\nu=100111011001010011100 \cdots
$$


Sendo assim, temos:

$F\left(z_{0}, z_{1}\right)=f^{S_{0}}\left(z_{0}, z_{1}\right)=\left(c, \hat{z}_{0}\right), F\left(z_{1}, z_{2}\right)=f^{S_{1}}\left(z_{1}, z_{2}\right)=\left(z_{0}, c\right), F\left(z_{2}, z_{3}\right)=f^{S_{2}}\left(z_{2}, z_{3}\right)=\left(z_{1}, c\right)$, $F\left(z_{3}, z_{4}\right)=f^{S_{3}}\left(z_{3}, z_{4}\right)=\left(z_{2}, c\right), \cdots$ podemos visualizar a aplicação na figura 4.1

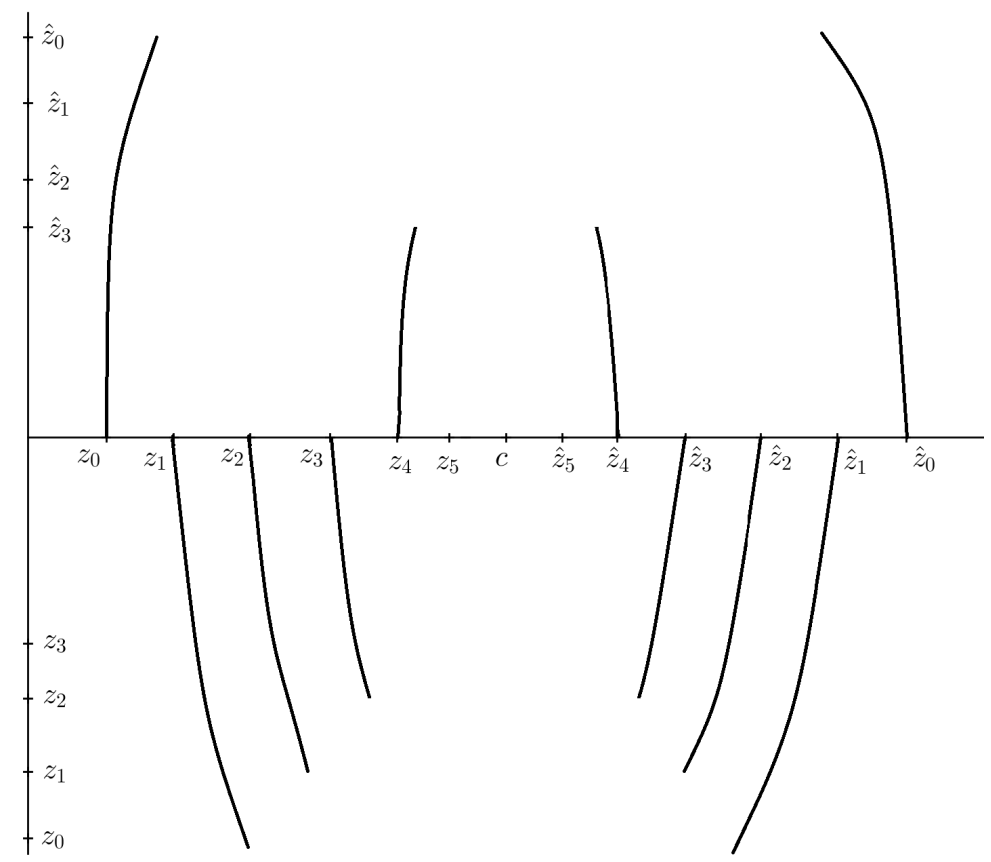

Figura 4.1: Aplicação induzida F para a aplicação de Fibonacci

Neste caso, vemos os domínios e as imagens ficarem menores cada vez mais, impossibilitando um bom controle de distorção e consequentemente, nos impedindo de garantir que a aplicação admita piac utilizando o teorema de Folclore.

(ii) A aplicação tenda, $T(x)=\min \{\sqrt{2} x, \sqrt{2}(1-x)\}$

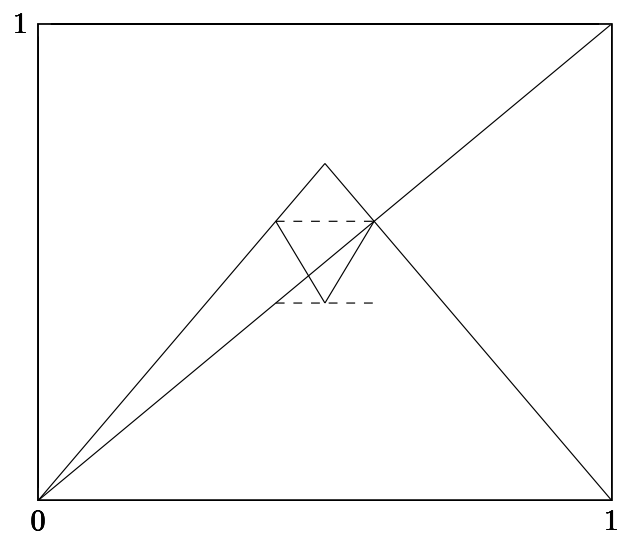

Figura 4.2: $T(x)=\min \{\sqrt{2} x, \sqrt{2}(1-x)\}$ 
A sequência Kneading desta aplicação é dada por:

$$
\nu=10111111111111 \cdots
$$

Neste caso, $F\left(A_{1}\right)=\left(c, \hat{z}_{0}\right)$ e para todo $k \geq 2$ temos $F\left(A_{k}\right)=\left(z_{1}, c\right)$ ou $F\left(A_{k}\right)=\left(c, \hat{z}_{1}\right)$. Com isso, apesar dos domínios ficarem cada vez menores, as imagens permanecem grandes, nos garantindo boas propriedades de distorção. Observamos também que esta aplicação induzida $F$ preserva a partição e $\left.F\right|_{A_{k}}$ é monótona para todo $k$, portanto é uma aplicação de Markov. Além disto, $\left|F^{n}\left(A_{k}\right)\right| \geq \beta$ para todo $n$ e todo ramo $\left.F^{n}\right|_{A_{k}}$. Sendo assim, podemos utilizar o teorema de Folclore 3.4.3 e concluir que $F$ admite piac.

Sendo assim, nosso interesse será construir uma aplicação induzida de forma que cada imagem de $F$ por $A_{k}$ permaneça sempre grande, isto é, $\left|z_{Q(k)}-c\right| \geq B>0$ a fim de nos garantir boas propriedades de distorção e que seja de Markov para podermos utilizar o Teorema de Folclore. Com isso, definimos as aplicações ramo-longo.

Uma aplicação $f$ é chamada de ramo-longo se existe $\beta>0$ tal que pra todo $n$ e todo intervalo de monotonicidade $T$ de $f^{n}$ temos $\left|f^{n}(T)\right| \geq \beta$.

Assim, veremos dois lemas bastante úteis para a construção do nosso resultado. O primeiro lema caracteriza as aplicações ramo-longo através da limitação da aplicação Kneading e o segundo nos fornece um espaço de Koebe a fim de garantir posteriormente que a distorção de cada ramo de $F^{n}$ é limitada.

Lema 4.1.2 Uma aplicação $f$ é uma aplicação ramo-longo se, e somente se, existir $B$ tal que $Q(k) \leq B$ para todo $k \in \mathbb{N}$.

\section{Demonstração:}

Suponha, a priori, que $T$ seja um intervalo de monotocidade de $f^{n}$ tal que $f^{n}(T) \ni c$ e que $Q(k) \leq B$. Por conseguinte, existem $0 \leq a<b<n$ tais que

- $f^{a}(T)=\left(z_{r-1}, c\right)\left(\right.$ ou $\left.\left(c, \hat{z}_{r-1}\right)\right)$

- $f^{b}(T)=\left(c, c_{S_{r-1}}\right)$

Com isso, $n=b+S_{Q(r)}$ e $f^{n}(T)=\left(c_{S_{Q(r)}}, c_{S_{r}}\right)$.

Pela proposição 2.2 .2 , temos $c_{S_{r}} \notin\left(z_{Q(r+1)}, \hat{z}_{Q(r+1)}\right)$. Logo, como $Q(r) \leq B$ para todo $r$ então $c_{S_{r}}$ não pode está próximo de $c$, então existe $B^{\prime}$ tal que $\left(z_{B^{\prime}}, \hat{z}_{B^{\prime}}\right) \subset f^{n}(T)$ sendo assim, temos $f$ é ramo-longo.

Caso não tenhamos $f^{n}(T) \ni c$, sabemos também que existem $0 \leq a<b<n$ tais que

- $f^{a}(T)=\left(z_{r-1}, c\right)\left(\right.$ ou $\left.\left(c, \hat{z}_{r-1}\right)\right)$

- $f^{b}(T)=\left(c, c_{S_{r-1}}\right)$

Pela proposição 2.2.2, sabemos que $c_{S_{r-1}} \notin\left(z_{Q(r)}, \hat{z}_{Q(r)}\right)$. Logo, supondo $Q(r) \leq B$ para todo $r$ então $c_{S_{r-1}}$ não pode está próximo de $c$, então existe $B^{\prime}$ tal que $\left(z_{B^{\prime}}, \hat{z}_{B^{\prime}}\right) \subset f^{b}(T)$, com isso, garantimos que $\rho<\left|c-c_{S_{r-1} \mid}\right|$. Contudo, sabemos que $b \leq n<b+S_{Q(r)}$, e para todo $n$ neste intervalo $c \notin f^{n}(T)$, sendo assim, temos $f$ ramo-longo.

Por outro lado, seja $f$ ramo-longo. Para todo $T$ um intervalo de monotonicidade de $f^{n}$ sabemos que existe $\beta>0$ tal que $\left|f^{n}(T)\right| \geq \beta$ para todo $n$. Se $f^{n}(T) \ni c$, sabemos ainda que para cada $T$ existe um $r$ tal que $f^{n}(T)=\left(c_{S_{Q(r)}}, c_{S_{r}}\right)$. Como $c_{S_{r}} \notin\left(z_{Q(r+1)}, \hat{z}_{Q(r+1)}\right), c_{S_{r}}$ não pode está próximo de $c$ e para um $B$ fixo temos $S_{Q(r)} \leq S_{B}$, consequentemente $Q(r) \leq B$. Caso $f^{n}(T) \not \supset c$, sabemos que para um $0<b \leq n$ temos $f^{b}(T)=\left(c, c_{S_{r-1}}\right)$ e com argumentos similares concluimos que $S_{Q(r)} \leq S_{B}$ 
Lema 4.1.3 Seja $f \in \mathcal{F}$ uma aplicação ramo-longo. Existe $\gamma>0$ tal que se $T$ é um intervalo de monotonicidade tal que $f^{n}(T) \ni z_{k}$ (respectivamente $\widehat{z}_{k}$ ), entâo $f^{n}(T) \supset\left(z_{k}-\gamma, z_{k}\right)$ (respectivamente $\left.\left(\widehat{z}_{k}, \widehat{z}_{k}+\gamma\right)\right)$.

\section{Demonstração:}

Como $f$ é uma aplicação ramo-longo, pelo lema anterior sabemos que existe um $B$ tal que $Q(k) \leq B$. Sem perda de generalidade, seja $T$ um intervalo de monotonicidade de $f^{n}$ tal que $f^{n}(T) \ni c$, por argumentos análogos a prova anterior sabemos que existem $0 \leq a<b<n$ tais que

- $f^{a}(T)=\left(z_{k-1}, c\right)\left(\right.$ ou $\left.\left(c, \hat{z}_{k-1}\right)\right)$

- $f^{b}(T)=\left(c, c_{S_{k-1}}\right)$

- $f^{n}(T)=\left(c_{S_{Q(k)}}, c_{S_{k}}\right)$

Neste caso, $b=a+S_{k-1}$ e $n=a+S_{k}$. Tome $z_{r} \in f^{n}(T)$. Se $r<B$ o resultado é imediato pois $Q(r) \leq B$, logo existe $\gamma>0$ tal que $f^{n}(T) \supset\left(z_{r}-\gamma, z_{r}\right)$. Sendo assim, suponha que $r \geq B$. Para garantirmos a existência do $\gamma>0$, chamemos $L=\max _{x}|D f(x)|$. Sabemos que $T$ contém um intervalo de monotonicidade $T^{\prime}$ de $f^{a+S_{k+2}}$. Como $f$ é ramo-longo, $\left|f^{a+S_{k+2}}\left(T^{\prime}\right)\right|>\beta$. Tomando a pré-imagem de $f^{S_{k+2}+a}\left(T^{\prime}\right)$ por um tempo limitado $S_{Q(k+2)}+S_{Q(k+1)}$ concluímos que

$$
\left[z_{Q(k+1)}, c_{S_{k}}\right] \supseteq f^{a+S_{k+2}-S_{Q(k+2)}-S_{Q(k+1)}}\left(T^{\prime}\right)>\frac{\beta}{L^{S_{Q(k+2)}+S_{Q(k+1)}}}=\gamma>0 .
$$

Podemos entender melhor observando a figura 4.3:



Figura 4.3: Aplicação $f^{n}$ para existência do $\gamma>0$

Aplicando a mesma ideia para o outro lado, concluímos que existe $\gamma>0$ tal que $f^{n}(T) \supset$ $\left(z_{k}-\gamma, z_{k}\right)$. 
Como $F\left(A_{k}\right)=\left(z_{Q(k)}, c\right)$ ou $F\left(A_{k}\right)=\left(c, \widehat{z}_{Q(k)}\right)$ observamos então que $F$ preserva a partição $\bigcup_{k} A_{k}$ de $\left(z_{0}, \widehat{z}_{0}\right)$ e com isso, $F$ é uma aplicação de Markov. Além disso, observamos que se $f$ é uma aplicação ramo-longo, então a aplicação induzida também é ramo-longo no lema a seguir.

Lema 4.1.4 Se f é uma aplicação ramo-longo, então a aplicação induzida também é ramo-longo, e existe $K>0$ tal que para todo $n \geq 0$, a distorção de todos os ramos de $F^{n}$ é limitada por $K$.

\section{Demonstração:}

Como $f$ é uma aplicação ramo-longo, sabemos que a aplicação Kneading é limitada. Como $F\left(A_{k}\right)=\left(z_{Q(k)}, c\right)$ ou $F\left(A_{k}\right)=\left(c, \widehat{z}_{Q(k)}\right)$ temos $F$ é ramo-longo. Como $F$ é uma aplicação de Markov, para calcularmos a distorção, tomemos $J$ algum ramo do domínio de algum iterado $F^{n}$, digamos $F^{n}(J)=f^{m}(J)=\left(z_{k}, c\right)$. Seja $T$ o intervalo de monotonicidade de $f^{m}$ contendo $J$.

Pelo lema 4.1.3, como $f^{m}(T) \ni z_{k}$, existe um $\gamma>0$ tal que $f^{m}(T)$ contém $\left(z_{k}-\gamma, z_{k}\right]$. Além disso, $f^{m}(T)$ também contém $\left[c, \hat{z}_{B}\right)$. Com isso, obtemos um espaço de Koebe. E pelo princípio de Koebe segue o resultado.

Pelo teorema de Folclore, percebemos que a aplicação induzida $F$ possui piac, contudo não garantimos ainda que $f$ também possua. A fim de garantir que $f$ possua piac, vemos a seguinte proposição.

Proposição 4.1.5 Seja $f \in \mathcal{F}$ uma aplicação de ramo-longo. Então $f$ admite piac se, e somente se, a condição de somatória

$$
\sum_{k} S_{k-1}\left|A_{k}\right|<\infty
$$

é satisfeita. Cada aplicação ramo-longo tem pelo menos uma medida invariante absolutamente continua $\sigma-f i n i t a$.

\section{Demonstração:}

Pelo Teorema de Folclore, sabemos que $F$ tem uma medida de probabilidade invariante $m$ que é absolutamente contínua com respeito à medida de Lebesgue. Definiremos a medida $\mu$ para a aplicação original $f$ da seguinte forma: para qualquer conjunto mensurável $A$ temos

$$
\mu(A)=\sum_{k \geq 1}^{\infty} \sum_{i=0}^{S_{k-1}-1} m\left(f^{-i}(A) \cap A_{k}\right) .
$$

Portanto, $\mu$ é absolutamente contínua pois se $|A|=0$ então $m(A)=0$ resultando em $\mu(A)=0$. Além disso, temos $\mu f$-invariante, como já vimos no lema 3.4.4. Sendo assim, $\mu\left(f^{-1}(A)\right)=\mu(A)$ e com isso,

$$
\mu(I)=\sum_{k \geq 0} \sum_{i=0}^{S_{k-1}-1} m\left(f^{-i}(I) \cap A_{k}\right) \leq \sum_{k \geq 0} S_{k-1} m\left(A_{k}\right) .
$$

Sabemos também pelo teorema de Folclore que $\frac{d m}{d x}$ é limitada. Seja $M$ o limite superior de $\frac{d m}{d x}$. Assim,

$$
m(I) \leq \sum_{k \geq 1} S_{k-1} M\left|A_{k}\right|
$$

Como cada ramo de $F$ cobre $\left(z_{B}, c\right)$ ou $\left(c, \hat{z}_{B}\right)$, existe então um $M^{\prime}$ tal que $m(A) \geq M^{\prime}|A|$ sempre que $A \subset\left(z_{B}, \hat{z}_{B}\right)$ for mensurável. Com isso,

$$
\sum_{k \geq B} S_{k-1} M^{\prime}\left|A_{k}\right| \leq m(I)
$$


Portanto, $\mu$ é infinita se, e somente se, $\sum_{k} S_{k-1}\left|A_{k}\right|=\infty$. Além disso, cada aplicação ramolongo tem pelo menos uma medida invariante absolutamente contínua $\sigma$-finita como podemos ver abaixo Sejam $U \subset\left(z_{B}, \hat{z}_{B}\right)$ um intervalo tal que

$$
\operatorname{orb}(c) \cap U=\emptyset
$$

e $V$ um intervalo tal que é o terço central de $U$ e assumimos que $f^{n}\left(A_{k}\right) \cap V \neq \emptyset$ para algum $0 \leq n<S_{k-1}$.

Seja $N$ um número natural tal que as componentes $U \backslash V$ contém um ponto de $\bigcup_{n \leq N} f^{-n}(c)$. Como $\left.f^{S_{k}}\right|_{\left(c, z_{k-1}\right)}$ é monótona segue que $S_{k-1}-n<N$. Assim

$$
\begin{aligned}
\mu(V) & =\sum_{j \geq 0} \sum_{i=0}^{S_{j-1}-1} m\left(f^{-i}(V) \cap A_{j}\right) \\
& \leq \sum_{j \geq 0} N m\left(A_{j}\right) \\
& \leq N .
\end{aligned}
$$

Pelo teorema 3.5.7, sabemos que $\bigcup_{n} f^{-n}(V)$ tem medida total. Sejam $V_{0}=V, V_{1}=f^{-1}\left(V_{0}\right) \backslash V_{0}$ e de maneira geral, $V_{j}=f^{-j}\left(V_{0}\right) \backslash \bigcup_{i=0}^{j-1} V_{i}$. Então podemos escrever $I=\bigcup_{n} V_{n} \cup W$ em que $W$ tem medida nula. Portanto,

$$
\mu\left(V_{j}\right) \leq \mu\left(f^{-j}(V)\right)=\mu(V)<\infty
$$

portanto, $\mu$ é de fato $\sigma$-finita.

\subsection{Retorno Sela-nó}

Chamaremos $c_{n}$ de retorno mais próximo de $c$ se não existe $c_{j}$ com $j<n$ tal que $c_{j} \in\left(\widehat{c}_{n}, c_{n}\right)$, em que $f\left(\widehat{c}_{n}\right)=f\left(c_{n}\right)$. Assim, chamaremos o retorno mais próximo de $c$ de retorno sela-nó se o ramo central é quase tangente a diagonal, vejamos na figura 4.4.

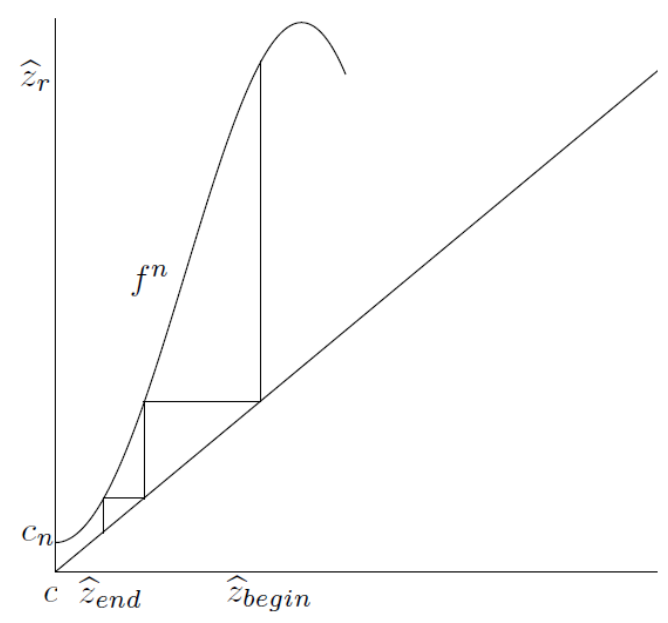

Figura 4.4: Retorno do tipo sela-nó 
Nos instantes $n$ em que $c$ é um retorno sela-nó, o ramo central de $f^{n}$ não cobre $c$ neste caso, então $n$ não é um tempo de corte. Seja $S_{k}$ o menor tempo de corte maior do que $n$. Então $f^{n}$ é monótona em $\left(c, \widehat{z}_{k-1}\right)$, e $f^{n}\left(\widehat{z}_{k}\right)=\widehat{z}_{r}$ para algum $r<k$, detotaremos $\widehat{z}_{k}=\widehat{z}_{\text {begin }}$. Se $f$ é uma aplicação ramo-longo, então $r \leq B$.

Voltando $\widehat{z}_{\text {begin }}$ ao longo do ramo central de $f^{n}$, teremos novos pontos pré-críticos mais próximos. Se $f_{n}$ é muito próximo da diagonal, estes pontos pré-críticos se aglomeram perto do funil. Passado do funil, as pré-imagens se separam novamente.

Chamaremos de $\widehat{z}_{\text {end }}$ a menor das pré-imagens para os quais $\left|\widehat{z}_{\text {end }}-c\right| \geq\left|c_{n}-c\right|$. A quantidade de pontos pré-críticos mais próximos entre $\widehat{z}_{\text {begin }}$ e $\widehat{z}_{\text {end }}$ pode ser arbitrariamente grande, desde que tomemos o ramo central de $f^{n}$ próximo da diagonal.

Para $t \in \mathbb{R}$, seja $x_{t} \in\left(c, \widehat{z}_{\text {begin }}\right)$ o ponto próximo de $c$ tal que $D f^{n}\left(x_{t}\right)=t$.

Lema 4.2.1 Sejam $f \in \mathcal{F}$ e $c_{n}$ um retorno sela-nó. Seja $\varepsilon=\left|\widehat{z}_{\text {begin }}-c\right|$, existem $K_{1}, K_{2}$ e $K_{3}$, independente de $n$, tal que

$$
K_{1} \varepsilon^{\ell /(\ell-1)} \leq\left|x_{2 \ell}-c\right| \leq K_{2} \varepsilon^{\ell /(\ell-1)},
$$

e se $r$ é o menor iterado tal que $f^{n r}\left(x_{2 \ell}\right) \notin\left(c, \widehat{z}_{\text {begin }}\right)$, então $r \leq K_{3} \log \log 1 / \varepsilon$.

\section{Demonstração:}

Escrevemos o ramo central $\left.f^{n}\right|_{(c, c+\varepsilon)}$ como sendo $\left.g \circ f\right|_{(c, c+\varepsilon)}$. Como $\left.f^{n}\right|_{(c, c+\varepsilon)}$ é monótona temos $\left.g\right|_{(f(c), f(c+\varepsilon))}$ também monótona, mas $(f(c), f(c+\varepsilon))$ não é o intervalo maximal em que $g$ é monótona. Assim, existe um intervalo de monotonicidade $T \supset(f(c), f(c+\varepsilon))$ de $g$ e chamemos $J=(f(c), f(c+\varepsilon))$.

Como $f^{n}\left(\widehat{z}_{\text {begin }}\right)=\widehat{z}_{r}$ por definição, resulta que $g(T) \ni \widehat{z}_{r}$. Com isso, pelo lema 4.1.3 existe um $\gamma$ tal que $g(T) \supset\left(\widehat{z}_{r}, \widehat{z}_{r}+\gamma\right)$.

Assim, sabemos que umas das componentes de $g(T \backslash J)$ sempre será maior ou igual a um $\delta_{1}|g(J)|$.

Por outro lado, afirmamos que $c \in g(T)$. De fato, sabemos que $g(T)=\left(c_{n-S_{k-1}}, c_{n-t}\right)$ e que $c_{n} \in g(T)$. Assim, se $c \notin g(T)$ então teremos que um ponto anterior ao $c_{n}$, sendo ele $c_{n-S_{k-1}}$ ou $c_{n-t}$, está mais próximo de $c$ do que o $c_{n}$, o que contradiz o fato de $c_{n}$ ser um retorno mais próximo de $c$. Logo, $c \in g(T)$.

Como, $c \in g(T)$ e $c_{n-p} \notin\left(\widehat{c}_{n}, c_{n}\right)$ para todo $0<p<n$, sabemos que existe um $\delta_{2}>0$ tal que a outra componente de $g(T \backslash J)$ sempre será maior ou igual a $\delta_{2}|g(J)|$.

Com isso, as componentes de $g(T \backslash J)$ sempre terão tamanho maior ou igual a $\delta|g(J)|$ em que $\delta=\min \left\{\delta_{1}, \delta_{2}\right\}$. E pelo Princípio de Koebe temos

$$
\operatorname{dis}(g, J) \leq K(\delta)
$$

Defina $\eta:=\left|f^{n}\left(\widehat{z}_{k}\right)-c_{n}\right|=\left|\widehat{z}_{r}-c_{n}\right|$ e $\rho:=\left|z_{B}-c\right|$. Como $f$ é ramo-longo, segue que existe um $\xi>0$ tal que

$$
1 \geq \eta \geq \frac{\left|z_{B}-c\right|}{\xi}
$$

Com isso,

$$
t=\left|D f^{n}\left(x_{t}\right)\right|=\left|D(g \circ f)\left(x_{t}\right)\right|=\left|D g\left(f\left(x_{t}\right)\right)\right| \cdot\left|D f\left(x_{t}\right)\right| .
$$

Por um lado, como a ordem crítica é $\ell$, temos

$$
\left|D f\left(x_{t}\right)\right| \leq \ell O_{2}\left|x_{t}-c\right|^{\ell-1} \quad \text { e } O_{1}\left|\widehat{z}_{k}-c\right|^{\ell} \leq\left|f\left(\widehat{z}_{k}\right)-f(c)\right|
$$

e

$$
\left|D g\left(f\left(x_{t}\right)\right)\right| \leq K \frac{\left|f^{n}\left(\widehat{z}_{k}\right)-f^{n}(c)\right|}{\left|f\left(\widehat{z}_{k}\right)-f(c)\right|} .
$$

Logo, como $\left|f^{n}\left(\widehat{z}_{k}\right)-f^{n}(c)\right| \leq 1$, concluímos que

$$
t=\left|D f^{n}\left(x_{t}\right)\right| \leq \frac{K \ell O_{2}\left|x_{t}-c\right|^{\ell-1}}{O_{1}\left|\widehat{z}_{k}-c\right|^{\ell}} .
$$


Por outro lado, como a ordem crítica é $\ell$, temos

$$
\ell O_{1}\left|x_{t}-c\right|^{\ell-1} \leq\left|D f\left(x_{t}\right)\right| \quad e \quad\left|f\left(\widehat{z}_{k}\right)-f(c)\right| \leq O_{2}\left|\widehat{z}_{k}-c\right|^{\ell}
$$

$\mathrm{e}$

$$
\frac{1}{K} \frac{\left|f^{n}\left(\widehat{z}_{k}\right)-f^{n}(c)\right|}{\left|f\left(\widehat{z}_{k}\right)-f(c)\right|} \leq\left|D g\left(f\left(x_{t}\right)\right)\right| .
$$

Assim,

$$
\frac{\ell O_{1} \rho\left|x_{t}-c\right|^{\ell-1}}{K \xi O_{2}\left|\widehat{z}_{k}-c\right|^{\ell}} \leq\left|D f^{n}\left(x_{t}\right)\right|=t
$$

e por (4.1) e (4.2) temos

$$
\frac{\ell O_{1} \rho\left|x_{t}-c\right|^{\ell-1}}{K \xi O_{2}\left|\widehat{z}_{k}-c\right|^{\ell}} \leq t=\left|D f^{n}\left(x_{t}\right)\right| \leq \frac{K \ell O_{2}\left|x_{t}-c\right|^{\ell-1}}{O_{1}\left|\widehat{z}_{k}-c\right|^{\ell}} .
$$

Portanto, tomando $t=2 \ell$, segue que

$$
\left(\frac{2 O_{1}}{K O_{2}}\right)^{\frac{1}{\ell-1}} \varepsilon^{\frac{\ell}{\ell-1}} \leq\left|x_{2 \ell}-c\right| \leq\left(\frac{2 K \xi O_{2}}{\rho O_{1}}\right)^{\frac{1}{\ell-1}} \varepsilon^{\frac{\ell}{\ell-1}},
$$

isto é, existem $K_{1}$ e $K_{2}$, independente de $n$, tais que

$$
K_{1} \varepsilon^{\frac{\ell}{\ell-1}} \leq\left|x_{2 \ell}-c\right| \leq K_{2} \varepsilon^{\frac{\ell}{\ell-1}}
$$

concluimos então a primeira parte do lema.

Agora, mostraremos a segunda parte do lema: Se $r$ é o menor iterado tal que $f^{n r}\left(x_{2 l}\right) \notin\left(c, \widehat{z}_{\text {begin }}\right)$, então $r \leq K_{3} \log \log 1 / \varepsilon$.

Como

$$
\frac{\ell O_{1} \rho|x-c|^{\ell-1}}{K \xi O_{2}\left|\widehat{z}_{k}-c\right|^{\ell}} \leq\left|D f^{n}(x)\right| \leq \frac{K \ell O_{2}|x-c|^{\ell-1}}{O_{1}\left|\widehat{z}_{k}-c\right|^{\ell}},
$$

podemos integrar $D f^{n}$ a fim de obter uma aproximada para $f^{n}$ além disso devemos que escolher uma função aproximada abaixo da função $f^{n}$ original para obtermos um tempo de escape que seja válido para $f^{n}$. Assim, podemos reescalar $\left.f^{n}\right|_{(c, c+\varepsilon)}$ como sendo $\varphi:[0, \varepsilon] \rightarrow \mathbb{R}$ definida por

$$
\varphi(x)=\eta\left(\frac{x}{\varepsilon}\right)^{\ell}+C \varepsilon^{\frac{\ell}{\ell-1}}
$$

e como

$$
\left|x_{2 \ell}-c\right| \leq\left(\frac{2 K \xi O_{2}}{\rho O_{1}}\right)^{\frac{1}{\ell-1}} \varepsilon^{\frac{\ell}{\ell-1}}
$$

para esta função $\varphi$ segue que

$$
x_{2 \ell}=\left(\frac{2}{\eta}\right)^{\frac{1}{\ell-1}} \varepsilon^{\frac{\ell}{\ell-1}}:=d_{0} \varepsilon^{\frac{\ell}{\ell-1}} .
$$

Assim, sabemos que

$$
\begin{aligned}
\varphi\left(d_{0} \varepsilon^{\frac{\ell}{\ell-1}}\right) & =\eta\left(\frac{d_{0} \varepsilon^{\frac{\ell}{\ell-1}}}{\varepsilon}\right)^{\ell}+C \varepsilon^{\frac{\ell}{\ell-1}} \\
& =\left(\eta d_{0}^{\ell}+C\right) \varepsilon^{\frac{\ell}{\ell-1}} \\
& =d_{1} \varepsilon^{\frac{\ell}{\ell-1}}
\end{aligned}
$$

Com isso, vamos calcular o $r$-ésimo iterado $\varphi$ aplicado no ponto $x_{2 \ell}$ com o objetivo de encon- 
tramos o tempo de escape, para assim concluirmos a segunda parte da prova.

De maneira geral, sabemos que

$$
\varphi^{r}\left(d_{0} \varepsilon^{\frac{\ell}{\ell-1}}\right)=\left(\eta d_{r-1}^{\ell}+C\right) \varepsilon^{\frac{\ell}{\ell-1}}:=d_{r} \varepsilon^{\frac{\ell}{\ell-1}} .
$$

Vamos provar então que

$$
d_{r} \geq 2^{\left(\ell^{r-1}\right)} d_{0} .
$$

Faremos a prova por indução. Primeiro passo, $r=1$ :

$$
\begin{aligned}
d_{1} & =\eta d_{0}^{\ell}+C \\
& =\eta\left(\frac{2}{\eta}\right)^{\frac{\ell}{\ell-1}}+C \\
& =2\left(\frac{2}{\eta}\right)^{\frac{1}{\ell-1}}+C \\
& =2 d_{0}+C \\
& \geq 2 d_{0}
\end{aligned}
$$

Hipótese de indução:

$$
d_{r} \geq 2^{\left(\ell^{r-1}\right)} d_{0}
$$

Segundo passo, mostrar que é válido para $r+1$. Assim,

$$
\begin{aligned}
d_{r+1} & =\eta d_{r}^{\ell} \\
& \geq \eta\left(2^{\ell^{r-1}}\right)^{\ell} d_{0}^{\ell}+C \\
& =2^{\ell^{r}}\left(\eta d_{0}^{\ell}\right)+C \\
& =2^{\ell^{r}} 2 d_{0}+C \\
& \geq 2^{\ell^{r}} d_{0}
\end{aligned}
$$

Logo, pelo princípio de indução, temos

$$
d_{r} \geq 2^{\ell^{r-1}} d_{0} \text { para todo } r \in \mathbb{N}
$$

e com isso, segue que

$$
\eta \geq \varphi^{r}\left(x_{2 \ell}\right)=d_{r} \varepsilon^{\frac{\ell}{\ell-1}} \geq 2^{\ell^{r-1}}\left(\frac{2}{\eta}\right)^{\frac{1}{\ell-1}} \varepsilon^{\frac{\ell}{\ell-1}}
$$

isto é,

$$
\left(\frac{\eta}{\varepsilon}\right)^{\frac{\ell}{\ell-1}} \geq 2^{\ell^{r-1}}
$$

Em vista disso, obtemos

$$
\ell^{r-1} \leq \frac{\ell}{\ell-1} \cdot \frac{1}{\log 2} \cdot \log \frac{\eta}{\varepsilon}
$$

que acarreta em

$$
(r-1) \log \ell \leq \log \frac{\ell}{\ell-1}-\log \log 2+\log \log \frac{\eta}{\varepsilon},
$$

ou seja,

$$
\begin{aligned}
r & \leq \frac{1}{\log \ell}\left(\log \frac{\ell}{\ell-1}-\log \log 2+\log \log \frac{\eta}{\varepsilon}\right)+1 \\
& \leq K_{3} \log \log \frac{1}{\varepsilon} .
\end{aligned}
$$


No lema a seguir vamos explorar um outro aspecto importante, a inclinação do ponto fixo.

Lema 4.2.2 Seja $f \in \mathcal{F}$, e seja $p$ o ponto fixo de orientação reversa. Para cada $\varepsilon>0$, existe $N$ com a propriedade a seguir. Suponha que $n$ é um tempo de corte e $n+1$ um tempo de co-corte. Suponha também que $c_{j} \in(c, 1)$ para cada $n \leq j \leq m$. Então para cada $k$ tal que $n+N \leq S_{k} \leq m-N$,

$$
|D f(p)|^{-2 / \ell}-\varepsilon \leq \frac{\left|z_{k+1}-c\right|}{\left|z_{k}-c\right|} \leq|D f(p)|^{-2 / \ell}+\varepsilon .
$$

\section{Demonstração:}

Como $n$ é um tempo de corte e $n+1$ é um tempo de co-corte, $f^{n-1}\left(H_{n-1}\right) \supset\left(c, \hat{z}_{0}\right)$. Sejam

$$
c=q_{0}<q_{2}<q_{4}<\cdots<p<\cdots<q_{5}<q_{3}<q_{1}=\hat{z}_{0}
$$

pontos pré-críticos próximos de $p$. Em particular,

$$
f\left(q_{i+1}\right)=q_{i}
$$

para cada $i \geq 0$.

Pelo Teorema do Valor Médio e pela continuidade da derivada segue que

$$
\frac{\left|q_{i+2}-p\right|}{\left|q_{i}-p\right|} \stackrel{i \rightarrow \infty}{\longrightarrow} \frac{1}{|D f(p)|^{2}}
$$

Seja $r=m-n$. Como $c_{j} \in(c, 1)$ para todo $n \leq j \leq m, c_{n}$ deve estar contido em $\left(q_{r}, q_{r-1}\right)$.

Além disso, os pontos $z_{k}$, com $n \leq S_{k} \leq m$, devem estar aplicados em $q_{0}, q_{2}$, etc. Seja $\varepsilon>0$ arbitrário. Então existe $N$, independente de $n$ e $m$, tal que por (4.3), para todo $i \geq N$ temos

$$
\left|\frac{\left|q_{i+2}-p\right|}{\left|q_{i}-p\right|}-\frac{1}{|D f(p)|^{2}}\right| \leq \frac{\varepsilon}{10}
$$

Para cada $i \leq r-N$,

$$
\left|\frac{\left|q_{i}-c_{n}\right|}{\left|q_{i}-p\right|}-1\right| \leq \frac{\varepsilon}{10}
$$

Sendo assim, como

$\left|\frac{\left|q_{i+2}-p\right|}{\left|q_{i}-p\right|}-\frac{1}{|D f(p)|^{2}}\right|=\left|\frac{\left|q_{i+2}-p\right|}{\left|q_{i}-p\right|} \cdot \frac{\left|q_{i+2}-c_{n}\right|}{\left|q_{i+2}-c_{n}\right|} \cdot \frac{\left|q_{i}-c_{n}\right|}{\left|q_{i}-c_{n}\right|}-\frac{1}{|D f(p)|^{2}}\right| \leq \frac{\varepsilon}{10}$ para $i \geq N$.

Por possuirmos $\left|\frac{\left|q_{i}-c_{n}\right|}{\left|q_{i}-p\right|}-1\right| \leq \frac{\varepsilon}{10}$ para $i \leq r-N$, temos também que $\left|\frac{\left|q_{i}-p\right|}{\left|q_{i}-c_{n}\right|}-1\right| \leq \frac{\varepsilon}{10}$ para $i \leq r-N$.

Com isso, é possível concluir que para cada $N \leq i \leq r-N$

$$
\left|\frac{\left|q_{i+2}-c_{n}\right|}{\left|q_{i}-c_{n}\right|}-\frac{1}{|D f(p)|^{2}}\right| \leq \frac{3 \varepsilon}{10} \leq \frac{\varepsilon}{3}
$$

A distorção de $\left.f^{n-1}\right|_{H}$ é limitada por $1+\left(\frac{\varepsilon}{10}\right)$, onde $c_{1} \in H \subset H_{n-1}\left(c_{1}\right)$ é o intervalo que é levado em $\left(q_{N}, q_{N-1}\right)$. Segue que os pontos $f\left(z_{k}\right), f\left(z_{k+1}\right), \cdots$, para $n+N \leq S_{k}<S_{k+1}<\cdots \leq m-N$, se acumulam em $f(c)$ com taxa exponencial aproximadamente $|D f(p)|^{-2}$.

Assim, para todo $x, y \in f^{-1}(H) \backslash\{c\}$ por ter ordem crítica $\ell$, ficamos com

$$
\left|\frac{|f(x)-f(c)|}{|x-c|^{\ell}} \cdot \frac{|y-c|^{\ell}}{|f(y)-f(c)|}-1\right| \leq \frac{\varepsilon}{10}
$$

Concluimos assim a demonstração. 


\subsection{Construção do algoritmo da sequência kneading}

A partir de agora, vamos contruir uma sequência kneading de uma aplicação unimodal de forma que esta seja ramo-longo e que garanta a existência de cascatas de bifurcação sela-nó. O algoritmo é dado da seguinte forma

(i) Seja $k=1, y_{k}=y_{1}=3$ e $\nu_{1} \nu_{2} \nu_{3}=101$.

(ii) Considere a concatenação $\nu_{1} \cdots \nu_{y_{k}} \nu_{1} \cdots \nu_{y_{k}} \nu_{1} \cdots \nu_{y_{k}} \cdots$ e escolha $w_{k}$ arbitrariamente mas de forma que a $w_{k}$-ésima e a $w_{k+3}$-ésima entradas da concatenação são zeros. Seja $\nu_{1} \cdots \nu_{w_{k}}$ a cadeia que consiste das primeiras $w_{k}$ entradas da concatenação.

(iii) Aumente $k$ por 1.

(iv) Seja $y_{k}>w_{k-1}+3$ arbitrário, tal que $y_{k}-w_{k-1}$ é ímpar. Seja $\nu_{1} \cdots \nu_{y_{k}}$ a concatenação de $\nu_{1} \cdots \nu_{w_{k-1}}$ e a cadeia de $y_{k}-w_{k-1}$ uns.

(v) Vá para a etapa $(i i)$.

Podemos verificar uma consequência importante da construção deste algoritmo da sequência kneading na proposição abaixo.

Proposição 4.3.1 Sejam $S_{k}$ e $S_{k-1}$ tempos de corte. Então, de acordo com a construção do algoritmo da sequência kneading, temos $S_{k}-S_{k-1} \in\{1,2\}$ para qualquer $k \in \mathbb{N}$.

\section{Demonstração:}

Pelo algoritmo dado sabemos que nossa sequência $\nu=\nu_{1} \nu_{2} \nu_{3} \cdots$ inicia com 101. Consequentemente $S_{0}=1$ e $S_{1}=2$. Para provarmos que

$$
S_{k}-S_{k-1} \in\{1,2\} \quad \forall k \in \mathbb{N}
$$

faremos uma prova por indução. Primeiro passo, tomemos $k=1$. Assim,

$$
S_{1}-S_{0}=1
$$

Hipótese de indução:

$$
S_{k}-S_{k-1} \in\{1,2\} .
$$

Segundo passo, devemos mostrar que

$$
S_{k+1}-S_{k} \in\{1,2\},
$$

ou seja, ou $S_{k+1}=S_{k}+1$ ou $S_{k+1}=S_{k}+2$. Por definição, temos

$$
S_{k+1}=\min \left\{n>S_{k} \mid \nu_{n} \neq \nu_{n-S_{k}}\right\}
$$

Suponha inicialmente que $\nu_{S_{k}}=0$. Pela construção da sequência kneading, existe no máximo um zero a cada três entradas consecutivas, $\operatorname{logo} \nu_{S_{k}+1}=1$ e $\nu_{S_{k}+2}=1$. Ou seja, $S_{k}+2$ é um tempo de corte.

Suponha agora que $\nu_{S_{k}}=1$. Se $\nu_{S_{k}+1}=0$ então $S_{k}+1$ é tempo de corte. Contudo, se $\nu_{S_{k}+1}=1$ devemos provar que $\nu_{S_{k}+2}=1$.

Suponha por absurdo que $\nu_{S_{k}+1}=1$ e $\nu_{S_{k}+2}=\nu_{2}=0$. Como $S_{k}$ é tempo de corte e $\nu_{S_{k}}=1$ temos $1=\nu_{S_{k}} \neq \nu_{2}=0$ e com isso $\nu_{S_{k}-1}=\nu_{1}=1$, isto é, $S_{k}-1$ não é um tempo de corte.

Como $S_{k}-S_{k-1} \in\{1,2\}$ temos $S_{k}-2$ tempo de corte.

Se $\nu_{S_{k}-2}=0$ então chegamos numa contradição devido a construção da sequência kneading, pois sabemos que $y_{k}>w_{k-1}+3$ e a posição $S_{k}$ é um $y_{j}$ para algum $j$. 
Se $\nu_{S_{k}-2}=1$ então concluimos que $\nu_{S_{k}-3}=1$ novamente chegariamos numa contradição da contrução da kneading ao analisar $\nu_{S_{k}-4}$ pois sabemos que $y_{k}-w_{k-1}$ é ímpar.

Portanto, se $\nu_{S_{k}+1}=1$ então $\nu_{S_{k}+2}=1$. Isto é, $S_{k+1}-S_{k} \in\{1,2\}$.

Logo, pelo princípio de indução, $S_{k}-S_{k-1} \in\{1,2\} \forall k \in \mathbb{N}$.

Através desta proposição percebemos que $S_{Q(k)} \in\{1,2\}$, isto é, existe um $B$ tal que $Q(k) \leq B$ $\forall k \in \mathbb{N}$. Pelo lema 4.1.2 sabemos que a aplicação unimodal $f$ é ramo-longo.

Exemplo 4.3.2 A sequência

$$
\nu(c)=1011011011011111 \cdots
$$

é um exemplo de início de uma sequência kneading que seguem os passos da construção do algoritmo definido anteriormente.



Figura 4.5: Aplicação induzida F para $\nu(c)=1011011011011111 \cdots$

Além deste resultado, podemos obter outros lemas interessantes diante da construção da sequência Kneading

Lema 4.3.3 Se $S_{j}<\tau_{i}<S_{j+1}$ então $\tau_{i}$ é único e $\tau_{i}=S_{j}+1$.

\section{Demonstração:}

Sabemos que $\tau_{k} \neq S_{r}$ para quaisquer que sejam $k$ e $r$, devido a sua definição.

Assim, pela proposição 4.3.1 temos $S_{k}-S_{k-1} \in\{1,2\}$ e como $S_{j}<\tau_{i}<S_{j+1}$ então $S_{j}=\tau_{i}-1$.

A unicidade de $\tau_{i}$ vem devido a construção dos números naturais.

Lema 4.3.4 Defina $w_{0}:=0$. Para cada $k \in \mathbb{N}$ resulta que $y_{k}-2 i$ é tempo co-corte para cada $0 \leq i \leq \frac{y_{k}-w_{k-1}-3}{2}$. 


\section{Demonstração:}

Faremos uma prova por indução. Primeiro passo, tomemos $k=1$ (devemos verificar que $y_{1}-2 i$ é um tempo de co-corte, em que $\left.0 \leq i \leq \frac{y_{1}-w_{0}-3}{2}\right)$.

Pela construção da sequência kneading, sabememos que $y_{1}=3$ e como por definição $w_{0}=0$, temos apenas o caso em que $i=0$. Portanto, $y_{1}-2 i=y_{1}=3=\min \left\{n>1 \mid \nu_{n}=1\right\}=\tau_{0}$.

Hipótese de indução: $y_{k}-2 i$ é um tempo de co-corte, para cada $0 \leq i \leq \frac{y_{k}-w_{k-1}-3}{2}$.

Segundo passo, mostrar que $y_{k+1}-2 i$ onde $0 \leq i \leq \frac{y_{k+1}-w_{k}-3}{2}$ é tempo co-corte.

Sabemos que $y_{k+1}-w_{k}$ é ímpar e que $y_{k+1}>w_{k}+3$ logo,

$$
\frac{y_{k+1}-w_{k}-3}{2} \in \mathbb{N}
$$

Como assumimos válido para $k$, nosso último tempo de co-corte é $y_{k}$. Logo, o próximo será o $\min \left\{n>y_{k} \mid \nu_{n} \neq \nu_{n-y_{k}}\right\}$. Devido a construção da kneading, vamos concatenar a sequência $\nu_{1} \cdots \nu_{y_{k}}$ até a escolha do $w_{k+1}$. Assim, só existirá a diferença entre $\nu_{n}$ e $\nu_{n-y_{k}}$ para $n=w_{k+1}+3$, como estaremos numa cadeia de uns, os demais tempos de co-corte ocorrerão em $w_{k+1}+5, \cdots, y_{k+1}$.

Com isso, temos válido para $k+1$. Logo, pelo princípio de indução, temos $y_{k}-2 i$ tempo de co-corte, onde $0 \leq i \leq \frac{y_{k}-w_{k-1}-3}{2}$, para cada $k \in \mathbb{N}$.

Proposição 4.3.5 Para todo $k \in \mathbb{N}$ temos ou $\tau_{k+1}-\tau_{k}=2$ ou $\tau_{k+1}-\tau_{k}=w_{k}+3-y_{k}$.

\section{Demonstração:}

Este resultado é uma consequência imediata do lema anterior, pois vimos que $\tau_{k}=y_{k}-2 i \mathrm{em}$ que $0 \leq i \leq \frac{y_{k}-w_{k-1}-3}{2}$. Assim, se $i=0$ então $\tau_{k}=y_{k}$ e $\tau_{k+1}=w_{k}+3$. Logo,

$$
\tau_{k+1}-\tau_{k}=w_{k}+3-y_{k}
$$

Se $i \neq 0$ então $\tau_{k}=w_{k-1}+3+2 j$ e $\tau_{k+1}=w_{k-1}+3+2(j+1)$ para algum $j$. Logo,

$$
\tau_{k+1}-\tau_{k}=2
$$

Portanto, temos válido o resultado.

Lema 4.3.6 Para cada inteiro $n$ tal que $\nu_{n}=0, n$ é um tempo de corte.

\section{Demonstração:}

Suponha que exista um inteiro $n$ tal que $\nu_{n}=0$ e que não seja um tempo de corte.

Assim, $\nu_{n-1}=1$ e $\nu_{n+1}=1$ por construção e como $S_{k}-S_{k-1} \in\{1,2\}$ devido a proposição 4.3.1 concluimos que $n-1$ e $n+1$ são tempos de corte.

Como $n-1$ é tempo de corte, o próximo tempo de corte é o $\min \left\{k>n-1 \mid \nu_{k} \neq \nu_{1}=1\right\}$.

Como $\nu_{n}=0$, temos $k=n$. Contradição, pois $n$ não é tempo de corte.

Portanto, para cada inteiro $n$ tal que $\nu_{n}=0$ temos um tempo de corte.

Lema 4.3.7 Para cada $k$, a sequência $\nu_{1} \nu_{2} \cdots \nu_{S_{k}}$ contém um número ímpar de uns.

\section{Demonstração:}

Para $k=0$, a sequência $\nu_{1} \nu_{2} \cdots \nu_{S_{0}}=\nu_{1}=1$. Logo, temos uma quantidade ímpar de uns. Assumimos o resultado válido para $k$, isto é, $\nu_{1} \nu_{2} \cdots \nu_{S_{k}}$ contém um número ímpar de uns.

Pela proposição 4.3 .1 sabemos que ou $S_{k+1}=S_{k}+1$ ou $S_{k+1}=S_{k}+2$. 
Se $S_{k+1}=S_{k}+1$ então pela construção da sequência kneading sabemos que $\nu_{S_{k}}=1$ e $\nu_{S_{k+1}}=0$, como até $\nu_{S_{k}}$ tinhamos uma quantidade ímpar de uns e $\nu_{S_{k+1}}=0$ mantivemos a quantidade ímpar de uns.

Se $S_{k+1}=S_{k}+2$ então pela construção da sequência kneading sabemos que $\nu_{S_{k}+1}=1 \mathrm{e}$ $\nu_{S_{k}+2}=1$, como até $\nu_{S_{k}}$ tinhamos uma quantidade ímpar de uns e como $\nu_{S_{k}+1}=1$ e $\nu_{S_{k}+2}=1$ mantivemos a quantidade ímpar de uns.

Logo, pelo princípio de indução concluimos que a sequência $\nu_{1} \nu_{2} \cdots \nu_{S_{k}}$ contém um número ímpar de uns, para todo $k$.

Lema 4.3.8 Para cada escolha de $\left\{y_{k}\right\}$ e $\left\{w_{k}\right\}$ construidos a partir do algoritmo a sequência Kneading $\nu$ resultante é admissivel, e corresponde a uma aplicação ramo-longo.

\section{Demonstração:}

Pela proposição 4.3.1 temos uma aplicação ramo-longo.

De acordo com [Bru94a], $\nu$ é admissível se a diferença $S_{k}-S_{k-1}$ e $\tau_{k}-\tau_{k-1}$ são todos tempos de corte.

Como $S_{k}-S_{k-1} \in\{1,2\}$ obtemos que esta diferença é um tempo de corte.

Pela proposição 4.3 .5 obtemos que ou $\tau_{k+1}-\tau_{k}=2$ ou $\tau_{k+1}-\tau_{k}=w_{k}+3-y_{k}$. Para $\tau_{k+1}-\tau_{k}=2$, sabemos que 2 é um tempo de corte. Para $\tau_{k+1}-\tau_{k}=w_{k}+3-y_{k}$ sabemos que $\nu_{w_{k}+3-y_{k}}=0 \mathrm{e}$ pelo lema 4.3.6 temos que $w_{k}+3-y_{k}$ é um tempo de corte.

\subsection{Demonstração do Teorema A}

Após termos construido todos pré-requisitos necessários para a prova do resultado procuraremos construir duas aplicações $f$ e $g$ topologicamente conjugadas tais que $\sum_{k} S_{k-1}\left|A_{k}(f)\right|<\infty$ e $\sum_{k} S_{k-1}\left|A_{k}(g)\right|=\infty$ pois vimos na proposição 4.1 .5 ao usarmos este critério mostraremos que $f$ possui medida de probabilidade invariante e absolutamente contínua enquanto $g$ não possui. Uma das ferramentas que vamos explorar é a diferença da inclinação do ponto crítico.

Teorema A. Existem aplicações unimodais $q_{\alpha}$ que possuem uma medida piac e são topologicamente conjugadas a aplicaçôes unimodais $f_{\beta}$ que não possuem medida piac.

\section{Demonstração:}

Tome $f, g \in \mathcal{F}$ conjugadas, tendo uma sequência Kneading como do tipo descrito no lema 4.3.8. Isso significa que ainda devemos determinar as sequências $y_{k}$ e $w_{k}$ e de forma que

$$
\left|D f\left(p_{f}\right)\right|>\left|D g\left(p_{g}\right)\right|,
$$

em que $p_{f}$ e $p_{g}$ denotam os pontos fixos de orientação reversa de $f$ e $g$ respectivamente.

Sendo assim, sejam $f_{a}=a \sin (\pi x)$ e $g_{b}=b x(1-x)$ duas famílias unimodais que, como vimos anteriormente, possuem derivada Schwarziana negativa.

Como é possível vermos em [dMvS93] para toda sequência Kneading $\nu$, existem $a$ e $b$ tais que $f_{a}$ e $g_{b}$ tem a mesma sequência Kneading $\nu$, e pelo teorema 2.1.4 estas funções serão topologicamente conjugadas. Além disso, sempre que $a \geq 0.92$ e $b \leq 3.83$ temos

$$
D f\left(p_{f}\right) \leq-1.85<-1.83 \leq D g\left(p_{g}\right)
$$

Portanto, existem $\lambda_{f}$ e $\lambda_{g}$ tais que, sob as condições do lema 4.2.2, 


$$
\frac{\left|z_{k+1}(f)-c\right|}{\left|z_{k}(f)-c\right|} \leq \lambda_{f}<\lambda_{g} \leq \frac{\left|z_{k+1}(g)-c\right|}{\left|z_{k}(g)-c\right|}
$$

Sendo assim, determinaremos as sequências $\left\{y_{k}\right\}$ e $\left\{w_{k}\right\}$ e consequentemente, estaremos definindo $\nu$. Por conseguinte, existem parâmetros $a \in[0.92,0.93]$ e $b \in[3.82,3.83]$, de maneira que $\nu\left(f_{a}\right)=\nu\left(g_{b}\right)=\nu$. De acordo com [GS97], $b$ é unicamente determinado.

Veremos que $f$ e $g$ exibirão uma sequência infinita de retornos do tipo sela-nó além dos iterados de $c$ ficarão constantemente muito próximos do ponto fixo.

Sejam então $\left\{a_{k}\right\}_{k \geq 1}$ e $\left\{b_{k}\right\}_{k \geq 1}$ sequências de inteiros correspondentes as sequências $\widehat{z}_{\text {begin }}$ e $\widehat{z}_{\text {end }}$ vistas anteriormente.

A escolha de $\left\{a_{k}\right\}_{k \geq 1}$ e $\left\{b_{k}\right\}_{k \geq 1}$ determina a escolha de $\left\{y_{k}\right\}$ e $\left\{w_{k}\right\}$ e vice-versa.

Podemos então, escolher $a_{k+1}>2 b_{k}+4 N$ tão grande que

(i) $\left|z_{a_{k+1}}(g)-c\right| \leq \frac{1}{2}\left|z_{b_{k}}(g)-c\right|$

(ii) $\left|z_{a_{k+1}}(f)-c\right| \leq \frac{1}{(k+1)^{2}}\left|z_{a_{k+1}}(g)-c\right|$

e podemos escolher o $b_{k+1}$ tão grande que

(iii) $1 \leq b_{k+1}\left|z_{a_{k+1}}(g)-c\right|^{\frac{\ell}{\ell-1}} \leq 2$.

Mostraremos que $\sum S_{i}\left|A_{i}(g)\right|=\infty$, isto é, que $g$ não admite medida de probabilidade invariante e absolutamente contínua. Por construção, sabemos que

$$
S_{i} \geq i
$$

para todo $i$. Com isso, prontamente obtemos

$$
\sum_{i} S_{i}\left|A_{i}(g)\right| \geq \sum_{i} i\left|A_{i}(g)\right|
$$

Além disso, percebemos que

$$
\begin{aligned}
\sum_{i=b_{k}}^{a_{k+1}} i\left|A_{i}\right| & \geq b_{k}\left|z_{b_{k}}-z_{b_{k}-1}\right|+\cdots+a_{k+1}\left|z_{a_{k+1}}-z_{a_{k+1}-1}\right| \\
& \geq b_{k}\left(\left|z_{b_{k}}-z_{b_{k}-1}\right|+\cdots+\left|z_{a_{k+1}}-z_{a_{k+1}-1}\right|\right) \\
& =b_{k}\left|z_{b_{k}-1}-z_{a_{k+1}}\right| \\
& \geq b_{k}\left|z_{b_{k}}-z_{a_{k+1}}\right|
\end{aligned}
$$

Sendo assim,

$$
\sum_{i} i\left|A_{i}(g)\right| \geq \sum_{k}\left(\sum_{i=b_{k}}^{a_{k+1}} i\left|A_{i}(g)\right|\right) \geq \sum_{k} b_{k}\left|z_{a_{k+1}}(g)-z_{b_{k}}(g)\right| .
$$


Ademais, por $(i)$ temos:

$$
\begin{aligned}
\left|z_{a_{k+1}}-z_{b_{k}}\right| & =\left|z_{a_{k+1}}-c+c-z_{b_{k}}\right| \\
& =\left|z_{b_{k}}-c\right|-\left|z_{a_{k+1}}-c\right| \\
& \geq\left|z_{b_{k}}-c\right|-\frac{1}{2}\left|z_{b_{k}}-c\right| \\
& =\frac{1}{2}\left|z_{b_{k}}-c\right|
\end{aligned}
$$

Através disso, obtemos

$$
\sum_{k} b_{k}\left|z_{a_{k+1}}(g)-z_{b_{k}}(g)\right| \geq \sum_{k} \frac{b_{k}}{2}\left|z_{b_{k}}(g)-c\right|
$$

Por (iii) e pelo lema 4.2.1, concluímos que

$$
\begin{aligned}
\sum_{i} S_{i}\left|A_{i}(g)\right| & \geq \sum_{k} \frac{b_{k}}{2}\left|z_{b_{k}}(g)-c\right| \\
& \geq \sum_{k} b_{k} \frac{\widetilde{K}_{1}}{2}\left|z_{a_{k}}(g)-c\right|^{\frac{\ell}{\ell-1}} \\
& =\frac{\widetilde{K}_{1}}{2} \sum_{k} b_{k}\left|z_{a_{k}}(g)-c\right|^{\frac{\ell}{\ell-1}} \\
& \geq \frac{\widetilde{K}_{1}}{2} \sum_{k} 1 \\
& =\infty
\end{aligned}
$$

Mostraremos agora que $\sum S_{i}\left|A_{i}(f)\right|<\infty$, isto é, que $f$ admite medida de probabilidade invariante absolutamente contínua.

Para $f$, seja $x_{k, 2 \ell} \in\left(c, \widehat{z}_{a_{k}}\right)$ o ponto próximo de c, tal que $D f^{n}\left(x_{k, 2 l}\right)=2 \ell$ (aqui, $n$ é o iterado correspondente do $k$-ésimo retorno sela-nó).

Pelo lema 4.2.1 temos

$$
\left|z_{b_{k}}-c\right| \leq\left|x_{k, 2 \ell}-c\right| \leq K_{2}\left|z_{a_{k}}-c\right|^{\frac{\ell}{\ell-1}} .
$$

Além disso, sabemos que se $r$ é o menor iterado tal que $f^{n r}\left(x_{k, 2 \ell}\right) \notin\left(c, \widehat{z}_{\text {begin }}\right)$ então

$$
r \leq K_{3} \log \log \frac{1}{\left|\widehat{z}_{\text {begin }}-c\right|}
$$

Como $\left|c-\hat{z}_{a_{k}}\right| \leq \lambda_{f}^{a_{k}-b_{k-1}-2 N}\left|c-\hat{z}_{b_{k-1}}\right| \leq \lambda_{f}^{\frac{a_{k}}{2}}\left|c-\hat{z}_{b_{k-1}}\right|$, o número de conjuntos $A_{i}$ disjuntos de $\left(\widehat{x}_{k, 2 \ell}, x_{k, 2 \ell}\right)$ é limitado por $K_{4} a_{k} \log a_{k}$, para algum $K_{4}$. Considere $C=C\left(K_{1}, K_{2}, K_{3}, K_{4}\right)$.

Sendo assim, como $f$ é uma aplicação ramo-longo existe um $B$ de forma que

$$
\sum_{i \geq b_{1}} S_{i}\left|A_{i}(f)\right| \leq S_{B} \sum_{i \geq b_{1}} i\left|A_{i}(f)\right|
$$


Podemos observar que no somatório acima começamos em $b_{1}$, contudo como vamos mostrar que o somatório converge, não perderemos a validade do resultado ao olharmos o somatório a partir de 1, pois estaremos acrescentando a soma um número finito de termos. Além disso, podemos observar que podemos separar $\sum_{i \geq b_{1}} i\left|A_{i}(f)\right|$ em três somas, de maneira conveniente, a fim de obtermos nosso resultado. Sendo assim, segue que

$$
S_{B} \sum_{i \geq b_{1}} i\left|A_{i}(f)\right| \leq S_{B} \sum_{k \geq 2}[\underbrace{\sum_{i=b_{k-1}}^{a_{k}} i\left|A_{i}(f)\right|}_{(1)}+\underbrace{\sum_{i=a_{k}}^{a_{k}+K_{4} a_{k} \log a_{k}} i\left|A_{i}(f)\right|}_{(2)}+\underbrace{\sum_{i=a_{k}+K_{4} a_{k} \log a_{k}}^{b_{k}} i\left|A_{i}(f)\right|}_{(3)}]
$$

Analizaremos cada um dos membros deste somatótio a fim de obtermos cotas por cima em função de $\left|z_{b_{k}}(f)-c\right|$ ou $\left|x_{2 \ell}-c\right|$.

Em (1) temos

$$
\begin{aligned}
\sum_{i=b_{k-1}}^{a_{k}} i\left|A_{i}(f)\right| & \leq \sum_{i=0}^{a_{k}-b_{k-1}}\left(i+b_{k-1}\right)\left|A_{i+b_{k-1}}(f)\right| \\
& \leq \sum_{i=0}^{a_{k}-b_{k-1}}\left(i+b_{k-1}\right) \lambda_{f}^{i-2 N}\left|z_{b_{k-1}}(f)-c\right|
\end{aligned}
$$

Em (2), como $\left|z_{a_{k}}(f)-c\right|$ é o maior intervalo dos somatório dos anéis e como $a_{k}+K_{4} a_{k} \log a_{k}$ é o maior número do somatório, obtemos

$$
\begin{aligned}
\sum_{i=a_{k}}^{a_{k}+K_{4} a_{k} \log a_{k}} i\left|A_{i}(f)\right| & \leq\left(a_{k}+K_{4} a_{k} \log a_{k}\right)\left|z_{a_{k}}(f)-c\right| \\
& \leq\left(a_{k}+K_{4} a_{k} \log a_{k}\right) \lambda_{f}^{a_{k}-b_{k-1}-2 N}\left|z_{b_{k-1}}(f)-c\right|
\end{aligned}
$$

Já em (3), devido a localização do $x_{k, 2 \ell}$, segue que

$$
\sum_{i=a_{k}+K_{4} a_{k} \log a_{k}}^{b_{k}} i\left|A_{i}(f)\right| \leq b_{k}\left|x_{k, 2 \ell}-c\right|
$$

Por conseguinte, através destas cotas encontradas em função de $\left|z_{b_{k}}(f)-c\right|$ e $\left|x_{k, 2 \ell}-c\right|$ e utilizando o fato de que $\left|z_{b_{k}}-c\right| \leq\left|x_{k, 2 \ell}-c\right| \leq K_{2}\left|z_{a_{k}}-c\right|^{\frac{\ell}{\ell-1}}$ temos:

$$
S_{B} \sum_{i \geq b_{1}} i\left|A_{i}(f)\right| \leq S_{B} \sum_{k \geq 1} C K_{2} b_{k}\left|z_{a_{k}}(f)-c\right|^{\frac{\ell}{\ell-1}} .
$$


Usando agora (ii) e (iii) concluímos que

$$
\begin{aligned}
\sum_{i \geq b_{1}} S_{i}\left|A_{i}(f)\right| & \leq S_{B} \sum_{k \geq 1} C K_{2} b_{k}\left|z_{a_{k}}(f)-c\right|^{\frac{\ell}{\ell-1}} \\
& \leq S_{B} C K_{2} \sum_{k \geq 1} \frac{1}{k^{2}} b_{k}\left|z_{a_{k}}(g)-c\right|^{\frac{\ell}{\ell-1}} \\
& \leq S_{B} C K_{2} \sum_{k \geq 1} \frac{2}{k^{2}} \\
& <\infty
\end{aligned}
$$

Como queríamos demonstrar.

A prova original deste resultado por ser encontrado em [Bru98]. 


\section{Capítulo 5}

\section{Conjunto de Cantor Absorvente}

Um aspecto importante em dinâmica é o estudo de conjuntos minimais. Neste capítulo, estaremos interessados no estudo da medida de Lebesgue dos conjuntos de Cantor minimais de funções unimodais, em especial, estaremos buscando condições de existência para conjuntos de Cantor minimais de medida Lebesgue nula cuja bacia tem medida de Lebesgue total, ou seja, condições de existência de um atrator métrico mas que não é um atrator topológico.

Segundo Guckenheimer [Guc79] para a parte topológica, e Blokh e Lyubich [LB86] para a parte métrica temos a seguinte classificação de atratores.

Classificação de atratores. Seja $f:[0,1] \rightarrow[0,1]$ uma aplicação $S$-unimodal com ordem crítica $\ell$. Então $f$ tem um único atrator topológico, que é um dos seguintes

(i) Uma órbita periódica atratora

(ii) A união de $n$ intervalos que são ciclicamente permutados pela $f$. Em cada um destes intervalos $f^{n}$ é topologicamente transitiva.

(iii) Um conjunto de Cantor em que $f$ age como uma máquina de somar. Este é o caso infinitamente renormalizável. Além disso, $f$ tem uma única métrica atratora, que pode ser do tipo $(i),(i i)$, (iii) ou

(iv) Um conjunto de Cantor, mas (iii) não se aplica: $f$ é finitamente renormalizável.

Um atrator do tipo (iv) é chamado de Conjunto de Cantor Absorvente, essa terminologia apareceu no trabalho do J. Guckenheimer e S. Johnson em [GJ90].

Observamos que as aplicações tendas são finitamente renormalizáveis, ou seja, possuem no máximo uma quantidade finita de intervalos periódicos. Enquanto que o conjunto $\omega$-limite do seu extremo local $c=1 / 2$ pode ser um conjunto de Cantor invariante e minimal. Porém a bacia deste conjunto é um conjunto magro (ou seja, união enumerável de conjuntos fechados de interior vazio) com medida de Lebesgue nula. Aplicações tendas $T_{\alpha} \operatorname{com} \alpha>1$, sempre possui uma medida piac.

Existem muitas publicações em que se foi suspeitado que uma aplicação $f: I \rightarrow I$, pelo menos $C^{3}$, não poderia ter um conjunto de Cantor absorvente. Devemos notar, no entanto, que, em 1992, Misha Lyubich e Folkert Tangerman fizeram estimativas computacionais, sugerindo que existem conjuntos de Cantor absorventes para aplicações de Fibonacci da forma $x \mapsto x^{6}+k$. Além disso, foram publicados vários resultados que comprovam que estes conjuntos não podem existir em alguns casos particulares como, por exemplo, no caso unimodal quadrático em [Lyu91] quando tomamos $\ell=2$.

Neste contexto, as aplicações unimodais que nos interessaremos são aplicações unimodais de Fibonacci, de classe $C^{3}$, com derivada Schwarziana negativa, ou simplesmente, aplicações de Fibonacci $S$-unimodais.

A recorrência da órbita crítica de Fibonacci apareceu no trabalho de Branner e Hubbard sobre polinômios cúbicos complexos [BH92] e no trabalho de Yoccoz [Yoc]. Em seguida, Mikhail Lyubich e John Milnor em [ML93] analisaram com bastante cuidado e rigor, propriedades da aplicação unimodal de Fibonacci. 
Sendo assim, nosso foco será mostrar o seguinte resultado

Teorema B. Se $g_{\gamma}$ é aplicação de Fibonacci e $\ell$ é suficientemente grande, o conjunto w-limite do seu ponto crítico é um conjunto de Cantor minimal de medida Lebesgue nula cuja bacia tem medida de Lebesgue total.

É interessante salientar que podemos obter a validade deste resultado para aplicações unimodais de Fibonacci apenas de classe $C^{2}$, como em [BKNvS96], tornando assim, o resultado mais geral. Todavia, com o objetivo apenas de simplificar as contas vamos requerer o caso em que estas aplicações unimodais sejam pelo menos de classe $C^{3}$ com derivada Schwarziana negativa e utilizaremos uma ferramanta bastante interessante para a conclusão deste trabalho: a torre de Hofbauer. Além desta ferramenta, exploraremos algumas estimativas úteis para o desenvolvimento desse capítulo, além de apresentarmos alguns resultados probabilísticos e ergódicos necessários para tal demonstração.

A fim de abreviar a notação, denotaremos

$$
f^{S_{n}}(c)=d_{n}
$$

\subsection{A Combinatória da aplicação de Fibonacci}

O primeiro indício de estudos da aplicação de Fibonacci apareceu numericamente no trabalho de Tsuda [Tsu85] e nesta seção, vamos conhecer algumas de suas propriedades. Definiremos $f$ como uma aplicação de Fibonacci se a sequência de tempos de corte, $S_{i}$, coincide com os números de Fibonacci: $S_{0}=1, S_{1}=2$ e

$$
S_{n}=S_{n-1}+S_{n-2}
$$

Como vimos no capítulo anterior, sabemos que a aplicação Kneading $Q: \mathbb{N} \rightarrow \mathbb{N}$ é definida por:

$$
S_{n}-S_{n-1}=S_{Q(n)} \text {. }
$$

Dessa maneira, observamos que

$$
S_{n}-S_{n-1}=S_{n-2}=S_{Q(n)}
$$

Com isso, quando estivermos trabalhando com aplicações de Fibonacci a aplicação Kneading é definida simplesmente por $Q(n)=\max \{n-2,0\}$.

Exemplo 5.1.1 Para cada ordem crítica $\ell$ existe pelo menos um $\lambda>0$ tal que $f:[0,1] \rightarrow[0,1]$ definida por

$$
f(x)=\lambda\left[1-|2 x-1|^{\ell}\right]
$$

é uma aplicação unimodal de Fibonacci com ponto crítico $c=\frac{1}{2}$.

Vejamos alguns resultados fundamentais nesse contexto.

Lema 5.1.2 Seja $f$ uma aplicação de Fibonacci. Então $d_{n} \in A_{n-1}=\left(z_{n-2}, z_{n-1}\right) \cup\left(\hat{z}_{n-1}, \hat{z}_{n-2}\right)$.

\section{Demonstração:}

Suponhamos, sem perda de generalidade, que $d_{n} \in(c, 1)$. Como

$$
S_{n+1}=S_{n}+S_{n-1}
$$

Segue que $f^{S_{n}}\left(z_{n+1}\right)=\hat{z}_{n-1}$ como podemos observar na figura 5.1

Agora, suponhamos por absurdo que $\hat{z}_{n-2} \in\left(\hat{z}_{n-1}, d_{n}\right)$. Como $f^{S_{n-1}}\left(z_{n-2}\right)=d_{n-3}$ obtemos uma contradição pois $d_{n+1}$ é um retorno mais próximo de $c$ do que $d_{n-3}$. Portanto, $d_{n} \in A_{n-1}$. 




Figura 5.1: Aplicação $f^{S_{n+1}}$ para $d_{n} \in A_{n-1}$

Como consequência deste lema, segue a seguinte proposição

Proposição 5.1.3 Seja $f$ uma aplicação de Fibonacci então $d_{n} \in\left(d_{n-1}, \widehat{d}_{n-1}\right)$ e $c_{i} \notin\left(d_{n-1}, \widehat{d}_{n-1}\right)$ para cada $0<i<S_{n}$.

\section{Demonstração:}

Segue do lema anterior.

Através da proposição anterior, conseguimos ter uma boa noção da localização dos retornos dos pontos críticos nos tempos de corte.

$$
d_{1}<d_{2}<d_{5}<d_{6}<\cdots<c<\cdots<d_{7}<d_{4}<d_{3}<d_{0} .
$$

A sequência Kneading da aplicação de Fibonacci é dada por:

$$
\nu_{f}(c)=1001110110010 \cdots
$$

Além disso, podemos obter algumas propriedades bastante interessantes

Proposição 5.1.4 A aplicação de Fibonacci $f:[0,1] \rightarrow[0,1]$ satisfaz as seguintes propriedades.

(i) $c_{S_{n}}$ e $c_{S_{n+2}}$ então em lados opostos de c para todo $n \geq 0$.

(ii) Se T é um intervalo maximal, onde $c \in \partial T$, tal que $\left.f^{S_{n}}\right|_{T}$ é monotóna, então $f^{S_{n}}(T)=$ $\left(d_{n}, d_{n-2}\right)$.

\section{Demonstração:}

(i) Para verificar que $c_{S_{n}}$ e $c_{S_{n+2}}$ estão em lados opostos de $c$ para todo $n \geq 0$ vamos verificar que $\nu_{S_{n}} \neq \nu_{S_{n+2}}$ para todo $n \geq 0$. Por definição,

$$
S_{n+2}=\min \left\{i>S_{n+1} \mid \nu_{i} \neq \nu_{i-S_{n+1}}\right\}
$$

Isto significa que $\nu_{S_{n+2}} \neq \nu_{S_{n+2}-S_{n+1}}$. Como $f$ é uma aplicação de Fibonacci, $S_{n+2}=S_{n+1}+S_{n}$. Portanto, concluimos que $\nu_{S_{n+2}} \neq \nu_{S_{n}}$. 
(ii) Como $c \in \partial T$ sabemos que $f^{S_{n}}(c)=d_{n}$. Além disso, como $T$ é um intervalo maximal tal que $\left.f^{S_{n}}\right|_{T}$ é monotóna sabemos que $z_{n-1} \in \partial T$ e como $f^{S_{n}}\left(z_{n-1}\right)=f^{S_{n-1}+S_{n-2}}\left(z_{n-1}\right)=d_{n-2}$. Portanto, $f^{S_{n}}(T)=\left(d_{n}, d_{n-2}\right)$

\subsection{Estimativas}

Nesta seção, calculamos algumas estimativas que nos ajudarão no desenvolvimento do resultado principal deste capítulo. Para obtermos tais estimativas, precisaremos apenas de alguns conceitos básicos como, por exemplo, o controle de distorção da razão cruzada e a condição da ordem crítica ser $\ell$.

Seja $f\left(t_{n}\right)$ o ponto extremo da direita do intervalo de monotonicidade de $f^{S_{n}-1}$ contendo $f(c)$. Vale ressaltar que $f\left(t_{n}\right)$ não é imagem de nenhum ponto de $\left(z_{n-1}, c\right)$. Além disso, temos $f^{S_{n-1}+S_{n-3}-1}\left(f\left(t_{n}\right)\right)=c$. Por conseguinte, este intervalo de monotonicidade é $\left(f\left(z_{n-1}\right), f\left(t_{n}\right)\right)$ e sua imagem por $f^{S_{n}-1}$ é $\left(d_{n-2}, d_{n-4}\right)$. Podemos verificar na figura 5.2.



Figura 5.2: Aplicação $f^{S_{n}}$ para estimativas

Além disso, observamos o seguinte fato

Observação 5.2.1 Seja $y_{n}=f^{S_{n}}\left(d_{n}\right)$. Como $f$ é uma aplicação de Fibonacci segue que $y_{n} \in$ $\left(d_{n+1}, z_{n-1}\right)$ ou $y_{n} \in\left(\hat{z}_{n-1}, \hat{d}_{n+1}\right)$. Consequentemente,

$$
y_{n} \in\left(d_{n+1}, d_{n}\right) ; \quad f\left(z_{n}\right) \in\left(f\left(d_{n+1}\right), f\left(d_{n+2}\right)\right)
$$

Tendo em vista esta observação, obtemos o seguinte lema

Lema 5.2.2 Seja $\lambda_{n}^{f}=\frac{\left|f\left(d_{n-2}\right)-f(c)\right|}{\left|f\left(d_{n}\right)-f(c)\right|}>1$ então para $n$ suficientemente grande $\lambda_{n}^{f}>3,86$ e $\ln \left(\frac{\left|f\left(d_{n-4}\right)-f(c)\right|}{\left|f\left(d_{n}\right)-f(c)\right|}\right)>2,7$.

\section{Demonstração:}

Sejam

- $l=\left(f\left(z_{n}\right), f\left(d_{n+2}\right)\right)$; 
- $j=\left(f\left(d_{n+2}\right), f(c)\right)$;

- $r=\left(f(c), f\left(t_{n}\right)\right)$

de tal modo que $t=l+j+r=\left(f\left(z_{n}\right), f\left(t_{n}\right)\right)$. Além disso, definamos também

- $L=\left(c, y_{n}\right)$;

- $J=\left(y_{n}, d_{n}\right)$;

- $R=\left(d_{n}, d_{n-4}\right)$

de maneira que $T=L+J+R=\left(c, d_{n-4}\right)$.

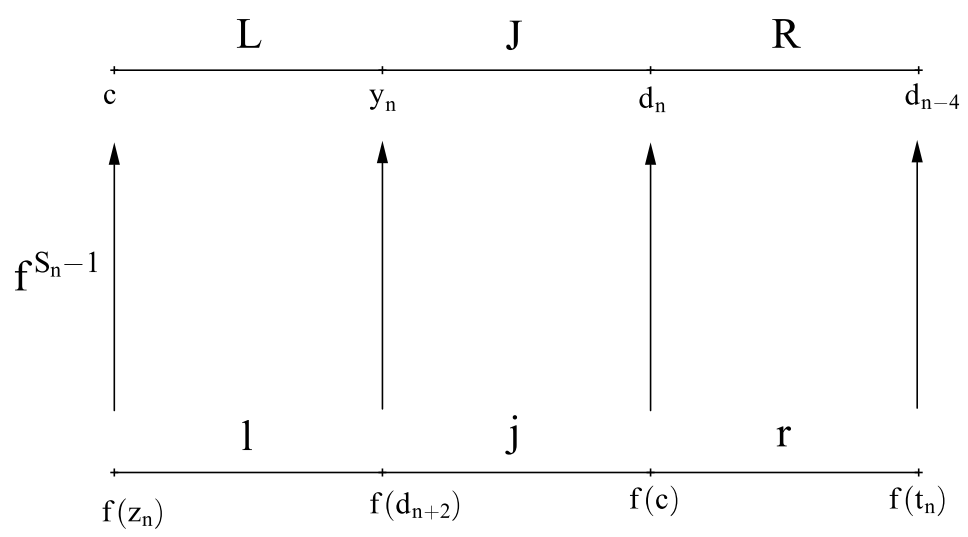

Figura 5.3: Aplicação $f^{S_{n}-1}$

Vimos que a composição de funções com derivadas de Schwarz negativa também possui derivada de Schwarz negativa. Com isso, concluímos pela proposição 3.2.5 que

$$
B\left(f^{S_{n}-1}, t, j\right) \geq 1
$$

Utilizando a Definição 3.2.3 que se refere a distorção da razão cruzada obtemos

$$
B\left(f^{S_{n}-1}, t, j\right)=\frac{D\left(f^{S_{n}-1}(t), f^{S_{n}-1}(j)\right)}{D(t, j)}=\frac{|J||T|}{|L||R|} \cdot \frac{|l||r|}{|t||j|} \geq 1
$$

Dessa maneira, vemos que

$$
\frac{|J||T|}{|j||t|} \geq \frac{|L||R|}{|l||r|}
$$

Portanto, ao substituirmos tais valores obtemos

$$
\frac{\left|d_{n}-y_{n}\right|\left|d_{n-4}-c\right|}{\left|f\left(d_{n+2}\right)-f(c)\right||t|} \geq \frac{\left|y_{n}-c\right|\left|d_{n-4}-d_{n}\right|}{\left|f\left(z_{n}\right)-f\left(d_{n+2}\right)\right||r|}
$$

Que nos garante que

$$
\frac{\left|d_{n-4}-d_{n}\right|}{\left|d_{n-4}-c\right|} \leq \frac{\left|d_{n}-y_{n}\right|\left|f\left(z_{n}\right)-f\left(d_{n+2}\right)\right||r|}{\left|f\left(d_{n+2}\right)-f(c)\right|\left|y_{n}-c\right||t|}
$$


Sabemos pelo Teorema do Valor Médio e pelo fato da aplicação $f$ ter ponto crítico de ordem $\ell$ que

$$
\begin{aligned}
\frac{1}{\ell} \cdot\left(1-\frac{\left|f\left(d_{n}\right)-f(c)\right|}{\left|f\left(d_{n-4}\right)-f(c)\right|}\right) & =\frac{1}{\ell} \cdot\left(\frac{\left|f\left(d_{n-4}\right)-f(c)\right|-\left|f\left(d_{n}\right)-f(c)\right|}{\left|f\left(d_{n-4}\right)-f(c)\right|}\right) \\
& =\frac{1}{\ell} \cdot \frac{\left|f\left(d_{n-4}\right)-f\left(d_{n}\right)\right|}{\left|f\left(d_{n-4}\right)-f(c)\right|} \\
& \leq \frac{1}{\ell} \cdot \frac{\left|D f\left(d_{n-4}\right)\right|\left|d_{n-4}-d_{n}\right|}{\left|f\left(d_{n-4}\right)-f(c)\right|} \\
& \leq \frac{O_{2}}{O_{1}} \cdot \frac{\left|d_{n-4}-d_{n}\right|}{\left|d_{n-4}-c\right|}
\end{aligned}
$$

Pela observação 5.2.1 e pela razão cruzada temos

$$
\begin{aligned}
\frac{O_{2}}{O_{1}} \cdot \frac{\left|d_{n-4}-d_{n}\right|}{\left|d_{n-4}-c\right|} & \leq \frac{O_{2}}{O_{1}} \cdot \frac{\left|d_{n}-y_{n}\right|}{\left|f\left(d_{n+2}\right)-f(c)\right|} \cdot \frac{|r|}{|t|} \cdot \frac{\left|f\left(z_{n}\right)-f\left(d_{n+2}\right)\right|}{\left|y_{n}-c\right|} \\
& \leq \frac{O_{2}}{O_{1}} \cdot \frac{\left|d_{n}-d_{n+1}\right|}{\left|d_{n+1}-c\right|} \cdot \frac{\left|f\left(d_{n+2}\right)-f\left(d_{n+1}\right)\right|}{\left|f\left(d_{n+2}\right)-f(c)\right|} \\
& =\frac{O_{2}}{O_{1}} \cdot \frac{\left|d_{n}-d_{n+1}\right|}{\left|d_{n+1}-c\right|} \cdot\left(\frac{\left|f\left(d_{n+1}\right)-f(c)\right|}{\left|f\left(d_{n+2}\right)-f(c)\right|}-1\right) \\
& \leq \frac{O_{2}}{O_{1}} \cdot \frac{\left|d_{n}-d_{n+1}\right|}{\ell \cdot \frac{O_{2}}{O_{1}} \cdot \frac{\left|f\left(d_{n+1}\right)-f(c)\right|}{\left|D f\left(d_{n+1}\right)\right|}} \cdot\left(\frac{\left|f\left(d_{n+1}\right)-f(c)\right|}{\left|f\left(d_{n+2}\right)-f(c)\right|}-1\right)
\end{aligned}
$$

Novamente pelo Teorema do Valor Médio e usando o fato que $(\sqrt{a b}-1)^{2} \geq(a-1)(b-1)$ ficamos com

$$
\begin{aligned}
\frac{1}{\ell} \cdot\left(1-\frac{\left|f\left(d_{n}\right)-f(c)\right|}{\left|f\left(d_{n-4}\right)-f(c)\right|}\right) & \leq\left(\frac{O_{2}}{O_{1}}\right)^{2} \cdot \frac{1}{\ell} \cdot \frac{\mid f\left(d_{n}\right)-f\left(d_{n+1} \mid\right.}{\left|f\left(d_{n+1}\right)-f(c)\right|} \cdot\left(\frac{\left|f\left(d_{n+1}\right)-f(c)\right|}{\left|f\left(d_{n+2}\right)-f(c)\right|}-1\right) \\
& \leq\left(\frac{O_{2}}{O_{1}}\right)^{2} \cdot \frac{1}{\ell} \cdot\left(\frac{\left|f\left(d_{n}\right)-f(c)\right|}{\left|f\left(d_{n+1}\right)-f(c)\right|}-1\right) \cdot\left(\frac{\left|f\left(d_{n+1}\right)-f(c)\right|}{\left|f\left(d_{n+2}\right)-f(c)\right|}-1\right) \\
& \leq\left(\frac{O_{2}}{O_{1}}\right)^{2} \cdot \frac{1}{\ell} \cdot\left(\sqrt{\frac{\left|f\left(d_{n}\right)-f(c)\right|}{\left|f\left(d_{n+2}\right)-f(c)\right|}-1}\right)^{2}
\end{aligned}
$$

Assim, como $\lambda_{n}^{f}=\frac{\left|f\left(d_{n-2}\right)-f(c)\right|}{\left|f\left(d_{n}\right)-f(c)\right|}$ segue que

$$
\left(\lambda_{n}^{f} \lambda_{n-2}^{f}\right)^{-1}=\frac{\left|f\left(d_{n}\right)-f(c)\right|}{\left|f\left(d_{n-4}\right)-f(c)\right|}
$$

Portanto, concluímos que

$$
1-\left(\lambda_{n}^{f} \lambda_{n-2}^{f}\right)^{-1} \leq\left(\frac{O_{2}}{O_{1}}\right)^{2} \cdot\left(\sqrt{\lambda_{n+2}^{f}}-1\right)^{2}
$$

Definimos $\lambda_{\infty}^{f}=\liminf \lambda_{n}^{f}$. 
Observando que $\frac{O_{2}}{O_{1}} \stackrel{n \rightarrow \infty}{\longrightarrow} 1$. Assim, quando $n \rightarrow \infty$ ficamos com

$$
2\left(\lambda_{\infty}^{f}\right)^{\frac{5}{2}}-\left(\lambda_{\infty}^{f}\right)^{3} \leq 1
$$

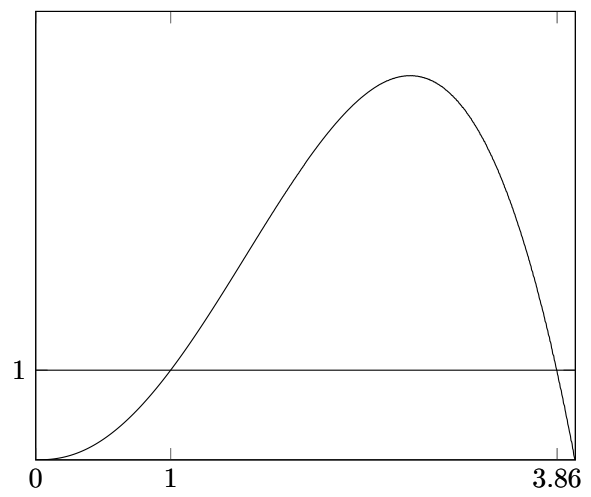

Figura 5.4: Aplicação $f(x)=2 x^{\frac{5}{2}}-x^{3}$

Como $\lambda_{n}^{f}=\frac{\left|f\left(d_{n-2}\right)-f(c)\right|}{\left|f\left(d_{n}\right)-f(c)\right|}>1$ concluímos que $\lambda_{\infty}^{f}>3,86$. Observe a figura 5.4. Com isso, obtemos que

$$
\ln \left(\frac{\left|f\left(d_{n-4}\right)-f(c)\right|}{\left|f\left(d_{n}\right)-f(c)\right|}\right)=\ln \left(\frac{\left|f\left(d_{n-2}\right)-f(c)\right|\left|f\left(d_{n-4}\right)-f(c)\right|}{\left|f\left(d_{n}\right)-f(c)\right|\left|f\left(d_{n-2}\right)-f(c)\right|}\right) \geq 2 \ln \lambda_{\infty}^{f}>2,7 .
$$

Para encontrarmos outras cotas necessárias, é importante sabermos que

Observação 5.2.3 Para todo $x>0$ temos

$$
\frac{x-1}{x} \leq \ln (x) \leq x-1
$$

Esta simples observação vai nos ajudar a encontrar algumas das cotas desejadas.

Lema 5.2.4 Sejam $a \in\left(z_{n}, \hat{z}_{n}\right)$ um ponto do intervalo de monotonicidade de $f^{S_{n}}, b=f^{S_{n}}(a)$ e $f(b)=f^{S_{n}}(f(a))$. Então para $n$ suficientemente grande

$\left|D f^{S_{n}}(f(a))\right| \leq \frac{|f(b)-f(c)|}{|f(a)-f(c)|} \cdot \ln \left(\frac{\left|f\left(d_{n-4}\right)-f(c)\right|}{|f(b)-f(c)|}\right) \cdot \ln \left(\frac{\left|f\left(d_{n}\right)-f(c)\right|}{|f(b)-f(c)|}\right) \cdot\left(\frac{\left|f\left(d_{n-4}\right)-f(c)\right|}{|f(b)-f(c)|}\right)^{\frac{1}{\ell}}$.

\section{Demonstração:}

Vamos usar a razão cruzada para $f^{S_{n}-1}$ com o intervalo $l$ reduzido ao ponto $f(a)$. Sendo assim, sejam

- $l=\{f(a)\} ;$

- $j=(f(a), f(c))$; 


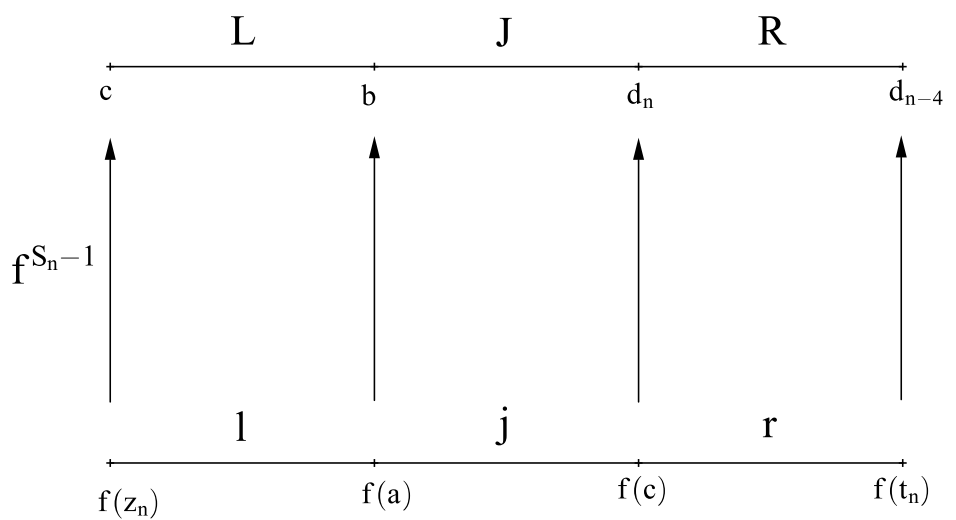

Figura 5.5: Aplicação $f^{S_{n}-1}$ para o cálculo de $\left|D f^{S_{n}}(f(a))\right|$

- $r=\left(f(c), f\left(t_{n}\right)\right)$

Sabemos que

$\left|D f^{S_{n}}(f(a))\right|=\left|D\left(f \circ f^{S_{n-1}}\right)(f(a))\right|=\left|D f\left(f^{S_{n}}(a)\right)\right|\left|D f^{S_{n-1}}(f(a))\right|=|D f(b)|\left|D f^{S_{n-1}}(f(a))\right|$

Assim, pelo fato da aplicação $f$ ter ponto crítico de ordem $\ell$ e pela razão cruzada temos

$$
\begin{aligned}
\left|D f^{S_{n}}(f(a))\right| & =|D f(b)|\left|D f^{S_{n-1}}(f(a))\right| \\
& \leq \frac{O_{2}}{O_{1}} \ell \frac{|f(b)-f(c)|}{|b-c|}\left|D f^{S_{n-1}}(f(a))\right| \\
& \leq \frac{O_{2}}{O_{1}} \ell \frac{|f(b)-f(c)|}{|b-c|} \cdot \frac{\left|d_{n}-b\right|}{|f(a)-f(c)|} \cdot \frac{\left|d_{n-4}-b\right|}{\left|d_{n-4}-d_{n}\right|} \\
& =\frac{O_{2}}{O_{1}} \frac{|f(b)-f(c)|}{|f(a)-f(c)|} \cdot \ell \frac{\left|d_{n}-b\right|}{\left|d_{n}-c\right|} \cdot \ell \frac{\left|d_{n-4}-b\right|}{\left|d_{n-4}-c\right|} \cdot \frac{1}{\ell \frac{\left|d_{n-4}-d_{n}\right|}{\left|d_{n}-c\right|}} \cdot \frac{\left|d_{n-4}-c\right|}{|b-c|} \\
& =\frac{O_{2}}{O_{1}} \frac{|f(b)-f(c)|}{|f(a)-f(c)|} \cdot \ell \frac{\frac{\left|d_{n}-c\right|}{|b-c|}-1}{\frac{\left|d_{n}-c\right|}{|b-c|}} \cdot \ell \frac{\frac{\left|d_{n-4}-c\right|}{|b-c|}-1}{\frac{\left|d_{n-4}-c\right|}{|b-c|}} \cdot \frac{1}{\ell\left(\frac{\left|d_{n-4}-c\right|}{\left|d_{n}-c\right|}-1\right)} \cdot \frac{\left|d_{n-4}-c\right|}{|b-c|}
\end{aligned}
$$

Pela observação 5.2.3 e novamente pelo fato da aplicação $f$ ter ponto crítico de ordem $\ell$, segue que

$$
\begin{aligned}
\left|D f^{S_{n}}(f(a))\right| & \leq \frac{O_{2}}{O_{1}} \frac{|f(b)-f(c)|}{|f(a)-f(c)|} \cdot \frac{\ln \left(\frac{\left|d_{n-4}-c\right|}{|b-c|}\right)^{\ell} \cdot \ln \left(\frac{\left|d_{n}-c\right|}{|b-c|}\right)^{\ell}}{\ln \left(\frac{\left|d_{n-4}-c\right|}{\left|d_{n}-c\right|}\right)^{\ell}} \cdot \frac{\left|d_{n-4}-c\right|}{|b-c|} \\
& \leq \frac{O_{2}}{O_{1}} \frac{|f(b)-f(c)|}{|f(a)-f(c)|} \frac{\ln \left(\frac{\left|f\left(d_{n-4}\right)-f(c)\right|}{|f(b)-f(c)|}\right) \cdot \ln \left(\frac{\left|f\left(d_{n}\right)-f(c)\right|}{|f(b)-f(c)|}\right)}{\ln \left(\frac{\left|f\left(d_{n-4}\right)-f(c)\right|}{\left|f\left(d_{n}\right)-f(c)\right|}\right)} \cdot\left(\frac{\left|f\left(d_{n-4}\right)-f(c)\right|}{|f(b)-f(c)|}\right)^{\frac{1}{\ell}} .
\end{aligned}
$$


Pelo lema 5.2.2 sabemos que $\ln \left(\frac{\left|f\left(d_{n-4}\right)-f(c)\right|}{\left|f\left(d_{n}\right)-f(c)\right|}\right)>2$. Sendo assim, segue o resultado.

Observação 5.2.5 A fim de simplificar a notação vamos introduzir

$$
\rho_{n}^{f}=\max \left\{\frac{\left|f\left(d_{k}\right)-f(c)\right|}{\left|f\left(d_{k+1}\right)-f(c)\right|} \mid n-N_{0} \leq k \leq n\right\},
$$

onde $N_{0} \leq 10$ podendo variar de um lema para outro. Suponha que b, como vimos no lema anterior, satisfaz $d_{n+i} \leq$ b para algum $i \leq 6$, então $\frac{\left|f\left(d_{n}\right)-f(c)\right|}{|f(b)-f(c)|} \leq\left(\rho_{n+i}^{f}\right)^{i}, \frac{\left|f\left(d_{n-4}\right)-f(c)\right|}{|f(b)-f(c)|} \leq\left(\rho_{n+i}^{f}\right)^{i+4} e$

$$
\left|D f^{S_{n}}(f(a))\right| \leq \frac{|f(b)-f(c)|}{|f(a)-f(c)|} \cdot(4+i) \cdot \ln \left(\rho_{n+i}^{f}\right) \cdot i \cdot \ln \left(\rho_{n+i}^{f}\right)\left(\rho_{n+i}^{f}\right)^{\frac{4+i}{\ell}} .
$$

Lema 5.2.6 Seja $\rho_{n}^{f}$ como definido anteriormente. Assim, obtemos a seguinte estimativa

$$
\left|D f^{S_{m}}\left(f\left(d_{m+1}\right)\right)\right| \leq 160 \cdot \frac{\left|f\left(d_{m+2}\right)-f(c)\right|}{\left|f\left(d_{m+1}\right)-f(c)\right|} \ln ^{4}\left(\rho_{m+2}^{f}\right) \cdot\left(\rho_{m+2}^{f}\right)^{\frac{13}{\ell}} .
$$

\section{Demonstração:}

Sabemos que $y_{m}=f^{S_{m}}\left(d_{m+2}\right)$. Com isso, $f\left(y_{m-1}\right)=f^{S_{m-1}}\left(f\left(d_{m+1}\right)\right)$. Portanto, podemos decompor $D f^{S_{m}}\left(f\left(d_{m+1}\right)\right)$ da seguinte forma

$$
\left|D f^{S_{m}}\left(f\left(d_{m+1}\right)\right)\right|=\left|D f^{S_{m-2}+S_{m-1}}\left(f\left(d_{m+1}\right)\right)\right|=\left|D f^{S_{m-2}}\left(f\left(y_{m-1}\right)\right)\right|\left|D f^{S_{m-1}}\left(f\left(d_{m+1}\right)\right)\right|
$$

Para achar uma cota por cima de $\left|D f^{S_{m-2}}\left(f\left(y_{m-1}\right)\right)\right|$ vamos utilizar o lema 5.2.4, colocando $n=m-2, a=y_{m-1}$ e $b=d_{m+2}$ e a observação 5.2 colocando $i=4$.

$$
\left|D f^{S_{m-2}}\left(f\left(y_{m-1}\right)\right)\right| \leq \frac{\left|f\left(d_{m+2}\right)-f(c)\right|}{\left|f\left(y_{m-1}\right)-f(c)\right|} \cdot 32 \cdot \ln ^{2}\left(\rho_{m+2}^{f}\right) \cdot\left(\rho_{m+2}^{f}\right)^{\frac{8}{\ell}} .
$$

Agora, para achar uma cota por cima de $\left|D f^{S_{m-1}}\left(f\left(d_{m+1}\right)\right)\right|$ vamos utilizar o lema 5.2.4, colocando $n=m-1, a=d_{m+1}$ e $b=y_{m-2}$ e como $d_{m} \in\left(y_{m-1}, \hat{y}_{m-1}\right), i=1$.

$$
\left|D f^{S_{m-1}}\left(f\left(d_{m+1}\right)\right)\right| \leq \frac{\left|f\left(y_{m-1}\right)-f(c)\right|}{\left|f\left(d_{m+1}\right)-f(c)\right|} \cdot 5 \cdot \ln ^{2}\left(\rho_{m}^{f}\right) \cdot\left(\rho_{m}^{f}\right)^{\frac{5}{\ell}} .
$$

Assim, o resultado segue tomando qualquer $\rho_{k}^{f}$, com $m-7 \leq k<m+2$.

O lema a seguir nos fornecerá cotas por cima e por baixo de $\left|D f^{S_{m}}(f(c))\right|$.

Lema 5.2.7 Para $m$ suficientemente grande, segue que

$$
\frac{\left|f\left(d_{m}\right)-f(c)\right|}{\left|f\left(d_{m+1}\right)-f(c)\right|}\left(\rho_{m}^{f}\right)^{-\frac{4}{\ell}} \leq\left|D f^{S_{m}}(f(c))\right| \leq 2 \cdot \frac{\left|f\left(d_{m}\right)-f(c)\right|}{\left|f\left(d_{m+2}\right)-f(c)\right|} \cdot \ln \left(\rho_{m+1}^{f}\right) \cdot\left(\rho_{m+1}^{f}\right)^{\frac{1}{\ell}} .
$$

\section{Demonstração:}

Para provar este resultado usaremos argumentos similares aos do lema 5.2.4.

Para obtermos a cota de $\left|D f^{S_{m}}(f(c))\right|$ por baixo usaremos a razão cruzada para $f^{S_{m}-1} \mathrm{em}$

- $l=\left(f\left(z_{m}\right), f(c)\right)$;

- $j=\{f(c)\}$;

- $r=\left(f(c), f\left(t_{n}\right)\right)$ 


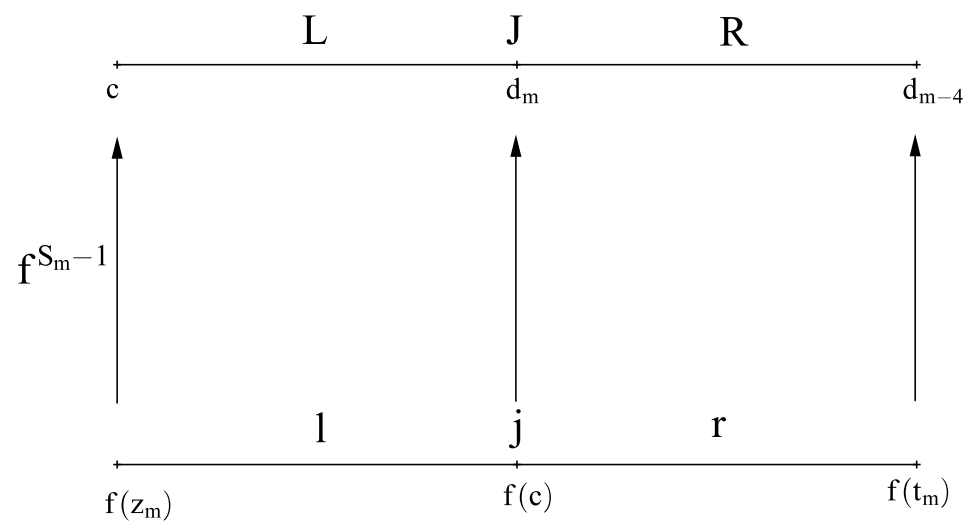

Figura 5.6: Aplicação $f^{S_{m}-1}$ para obter cota por baixo de $\left|D f^{S_{m}}(f(c))\right|$

Como podemos ver na figura 5.6.

Sabemos que

$$
\left|D f^{S_{m}}(f(c))\right|=\left|D\left(f \circ f^{S_{m-1}}\right)(f(c))\right|=\left|D f\left(d_{m}\right)\right|\left|D f^{S_{m-1}}(f(c))\right|
$$

Assim, pelo fato da aplicação $f$ ter ponto crítico de ordem $\ell$ e pela razão cruzada, segue que

$$
\begin{aligned}
\left|D f^{S_{m}}(f(c))\right| & \geq \frac{O_{1}}{O_{2}} \ell \frac{\left|f\left(d_{m}\right)-f(c)\right|}{\left|d_{m}-c\right|}\left|D f^{S_{m-1}}(f(c))\right| \\
& \geq \frac{O_{1}}{O_{2}} \frac{\left|f\left(d_{m}\right)-f(c)\right|}{\left|d_{m}-c\right|} \cdot \ell \frac{\left|d_{m-4}-d_{m}\right|}{\left|d_{m-4}-c\right|} \cdot \frac{\left|d_{m}-c\right|}{\left|f\left(z_{m}\right)-f(c)\right|} \\
& =\frac{O_{1}}{O_{2}} \ell\left(\frac{\left|d_{m-4}-c\right|}{\left|d_{m}-c\right|}-1\right) \cdot \frac{\left|d_{m}-c\right|}{\left|d_{m-4}-c\right|} \cdot \frac{\left|f\left(d_{m}\right)-f(c)\right|}{\left|f\left(z_{m}\right)-f(c)\right|}
\end{aligned}
$$

E utilizando observação 5.2.3 e pelo lema 5.2.2 temos

$$
\begin{aligned}
\left|D f^{S_{m}}(f(c))\right| & \geq \frac{O_{1}}{O_{2}} \ln \left(\frac{\left|d_{m-4}-c\right|}{\left|d_{m}-c\right|}\right)^{\ell} \cdot \frac{\left|d_{m}-c\right|}{\left|d_{m-4}-c\right|} \cdot \frac{\left|f\left(d_{m}\right)-f(c)\right|}{\left|f\left(z_{m}\right)-f(c)\right|} \\
& \geq \frac{O_{1}}{O_{2}} \ln \left(\frac{\left|f\left(d_{m-4}\right)-f(c)\right|}{\left|f\left(d_{m}\right)-c\right|}\right) \cdot\left(\frac{\left|f\left(d_{m}\right)-f(c)\right|}{\left|f\left(d_{m-4}\right)-f(c)\right|}\right)^{\frac{1}{\ell}} \cdot \frac{\left|f\left(d_{m}\right)-f(c)\right|}{\left|f\left(d_{m+1}\right)-f(c)\right|} \\
& \geq \frac{O_{1}}{O_{2}} \frac{\left|f\left(d_{m}\right)-f(c)\right|}{\left|f\left(d_{m+1}\right)-f(c)\right|} \cdot\left(\frac{\left|f\left(d_{m}\right)-f(c)\right|}{\left|f\left(d_{m-4}\right)-f(c)\right|}\right)^{\frac{1}{\ell}}
\end{aligned}
$$

Como

$$
\left(\frac{\left|f\left(d_{m}\right)-f(c)\right|}{\left|f\left(d_{m-4}\right)-f(c)\right|}\right)^{\frac{1}{\ell}}=\left(\frac{\left|f\left(d_{m-1}\right)-f(c)\right|}{\left|f\left(d_{m}\right)-f(c)\right|} \cdot \frac{\left|f\left(d_{m-2}\right)-f(c)\right|}{\left|f\left(d_{m-1}\right)-f(c)\right|} \cdot \frac{\left|f\left(d_{m-3}\right)-f(c)\right|}{\left|f\left(d_{m-2}\right)-f(c)\right|} \cdot \frac{\left|f\left(d_{m-4}\right)-f(c)\right|}{\left|f\left(d_{m-3}\right)-f(c)\right|}\right)^{-\frac{1}{\ell}}
$$

Concluimos que

$$
\frac{\left|f\left(d_{m}\right)-f(c)\right|}{\left|f\left(d_{m+1}\right)-f(c)\right|}\left(\rho_{m}^{f}\right)^{-\frac{4}{l}} \leq\left|D f^{S_{m}}(f(c))\right|
$$


Para obtermos a cota de $\left|D f^{S_{m}}(f(c))\right|$ por cima, usaremos a razão cruzada para $f^{S_{m}-1} \mathrm{em}$

- $l=\left(f\left(z_{m}\right), f\left(d_{m+2}\right)\right)$;

- $j=\left(f\left(d_{m+2}, f(c)\right)\right.$;

- $r=\{f(c)\}$

Como podemos ver na figura 5.7.

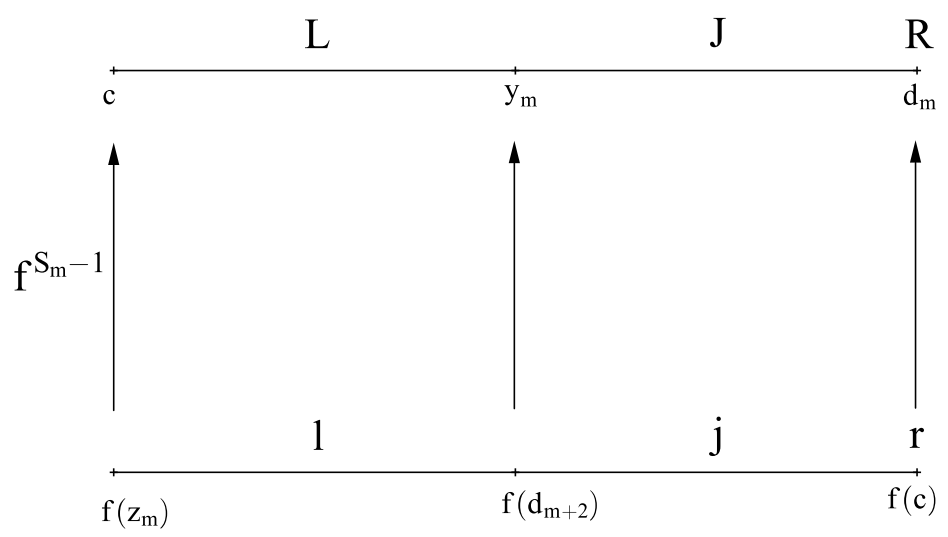

Figura 5.7: Aplicação $f^{S_{m}-1}$ para obter cota por cima de $\left|D f^{S_{m}}(f(c))\right|$

Assim, pelo fato da aplicação $f$ ter ponto crítico de ordem $\ell$ e pela razão cruzada segue que

$$
\begin{aligned}
\left|D f^{S_{m}}(f(c))\right| & =\left|D f\left(d_{m}\right)\right|\left|D f^{S_{m-1}}(f(c))\right| \\
& \leq \frac{O_{2}}{O_{1}} \ell \frac{\left|f\left(d_{m}\right)-f(c)\right|}{\left|d_{m}-c\right|}\left|D f^{S_{m-1}}(f(c))\right| \\
& \leq \frac{O_{2}}{O_{1}} \frac{\left|f\left(d_{m}\right)-f(c)\right|}{\left|d_{m}-c\right|} \cdot \ell \frac{\left|d_{m}-y_{m}\right|}{\left|f\left(d_{m+2}\right)-f(c)\right|} \cdot \frac{\left|d_{m}-c\right|}{\left|f\left(z_{m}\right)-f(c)\right|} \cdot \frac{\left|f\left(z_{m}\right)-f\left(d_{m+2}\right)\right|}{\left|y_{m}-c\right|}
\end{aligned}
$$

Pelas observações 5.2.1 e 5.2.3, e novamente pelo fato da aplicação $f$ ter ordem crítica $\ell$ temos

$$
\begin{aligned}
\left|D f^{S_{m}}(f(c))\right| & \leq \frac{O_{2}}{O_{1}} \frac{\left|f\left(d_{m}\right)-f(c)\right|}{\left|f\left(d_{m+2}\right)-f(c)\right|} \cdot \ell \frac{\left|d_{m}-d_{m+1}\right|}{\left|d_{m}-c\right|} \cdot \frac{\left|d_{m}-c\right|}{\left|d_{m+1}-c\right|} \\
& =\frac{O_{2}}{O_{1}} \frac{\left|f\left(d_{m}\right)-f(c)\right|}{\left|f\left(d_{m+2}\right)-f(c)\right|} \cdot \ell\left(\frac{\frac{\left|d_{m}-c\right|}{\left|d_{m+1}-c\right|}-1}{\frac{\left|d_{m}-c\right|}{\left|d_{m+1}-c\right|}}\right) \cdot \frac{\left|d_{m}-c\right|}{\left|d_{m+1}-c\right|} \\
& \leq \frac{O_{2}}{O_{1}} \frac{\left|f\left(d_{m}\right)-f(c)\right|}{\left|f\left(d_{m+2}\right)-f(c)\right|} \ln \left(\frac{\left|d_{m}-c\right|}{\left|d_{m+1}-c\right|}\right)^{\ell} \cdot \frac{\left|d_{m}-c\right|}{\left|d_{m+1}-c\right|} \\
& \leq \frac{O_{2}}{O_{1}} \frac{\left|f\left(d_{m}\right)-f(c)\right|}{\left|f\left(d_{m+2}\right)-f(c)\right|} \ln \left(\frac{\left|f\left(d_{m}\right)-f(c)\right|}{\left|f\left(d_{m+1}\right)-f(c)\right|}\right)\left(\frac{\left|f\left(d_{m}\right)-f(c)\right|}{\left|f\left(d_{m+1}\right)-f(c)\right|}\right)^{\frac{1}{\ell}} \\
& \leq \frac{O_{2}}{O_{1}} \frac{\left|f\left(d_{m}\right)-f(c)\right|}{\left|f\left(d_{m+2}\right)-f(c)\right|} \ln \left(\rho_{m+1}^{f}\right)\left(\rho_{m+1}^{f}\right)^{\frac{1}{\ell}}
\end{aligned}
$$


Assim, concluimos que

$$
\left|D f^{S_{m}}(f(c))\right| \leq \frac{\left|f\left(d_{m}\right)-f(c)\right|}{\left|f\left(d_{m+2}\right)-f(c)\right|} \cdot \ln \left(\rho_{m+1}^{f}\right) \cdot\left(\rho_{m+1}^{f}\right)^{\frac{1}{\ell}}
$$

Observação 5.2.8 Neste resultado poderíamos ter tomado $\rho^{f}$ como $\max \left\{\frac{\left|f\left(d_{k}\right)-f(c)\right|}{\left|f\left(d_{k+1}\right)-f(c)\right|} \mid m-4 \leq k \leq m\right\}$.

$\mathrm{Na}$ proposição a seguir ampliaremos a visão do lema anterior pensando no caso em que $n$ e a ordem crítica $\ell$ sejam suficientemente grandes.

Proposição 5.2.9 Para n e $\ell$ suficientemente grandes as derivadas $D f^{S_{n}}(f(c))$ e as razões $\frac{\left|f\left(d_{n}\right)-f(c)\right|}{\left|f\left(d_{n+1}\right)-f(c)\right|}$ são limitadas por cima e limitada por baixo por 1 mais uma constante que independente de $n e \ell$.

\section{Demonstração:}

Sabemos que

$$
\begin{aligned}
\left|D f^{S_{n}}(f(c))\right| & =\left|D f^{S_{n-2}+S_{n-1}}(f(c))\right| \\
& =\left|D f^{S_{n-2}}\left(f\left(d_{n-1}\right)\right)\right|\left|D f^{S_{n-1}}(f(c))\right| \\
& =\left|D f^{S_{n-2}}\left(f\left(d_{n-1}\right)\right)\right|\left|D f^{S_{n-3}+S_{n-2}}(f(c))\right| \\
& =\left|D f^{S_{n-2}}\left(f\left(d_{n-1}\right)\right)\right|\left|D f^{S_{n-3}}\left(f\left(d_{n-2}\right)\right)\right|\left|D f^{S_{n-2}}(f(c))\right|
\end{aligned}
$$

Usaremos o lema $5.2 .6 \mathrm{em}\left|D f^{S_{n-2}}\left(f\left(d_{n-1}\right)\right)\right|$ tomando $m=n-2$ e em $\left|D f^{S_{n-3}}\left(f\left(d_{n-2}\right)\right)\right|$ tomando $m=n-3$. Além disto, usaremos o lema $5.2 .7 \mathrm{em}\left|D f^{S_{n-2}}(f(c))\right|$ tomando $m=n-2$. Sendo assim, ficamos com

$$
\left|D f^{S_{n}}(f(c))\right| \leq 51200 \cdot \ln ^{9}\left(\rho_{n}^{f}\right) \cdot\left(\rho_{n}^{f}\right)^{\frac{27}{\ell}}
$$

Como sabemos pelo lema 5.2.7 que $\frac{\left|f\left(d_{m}\right)-f(c)\right|}{\left|f\left(d_{m+1}\right)-f(c)\right|}\left(\rho_{m}^{f}\right)^{-\frac{4}{\ell}} \leq\left|D f^{S_{m}}(f(c))\right|$, concluímos que

$$
\frac{\left|f\left(d_{m}\right)-f(c)\right|}{\left|f\left(d_{m+1}\right)-f(c)\right|} \leq 51200 \cdot \ln ^{9}\left(\rho_{n}^{f}\right) \cdot\left(\rho_{n}^{f}\right)^{\frac{31}{\ell}} .
$$

onde $\rho_{n}^{f}$ depende em quase 10 quocientes consecutivos $\frac{\left|f\left(d_{k}\right)-f(c)\right|}{\left|f\left(d_{k+1}\right)-f(c)\right|}$, $\operatorname{com} n-10 \leq k<n$. Isso nos fornece um limite superior do aumento de $\rho_{\infty}^{f}=\lim \sup \rho_{n}^{f}=\lim \sup \frac{\left|f\left(d_{n}\right)-f(c)\right|}{\left|f\left(d_{n+1}\right)-f(c)\right|}$ por

$$
\rho_{\infty}^{f} \leq 51200 \cdot \ln ^{9}\left(\rho_{\infty}^{f}\right) \cdot\left(\rho_{\infty}^{f}\right)^{\frac{31}{\ell}}
$$

(isto é, $\rho_{\infty}^{f}<10^{21}$ para $\ell$ grande) provando assim a primeira parte da proposição. Para obtermos o limite inferior usaremos as estimativas oriundas de lema 5.2.2. Assim, segue que

$$
\frac{\left|f\left(d_{m}\right)-f(c)\right|}{\left|f\left(d_{m+1}\right)-f(c)\right|} \geq 1+\frac{1-\exp ^{-2.7}}{\rho_{\infty}^{f}-1}
$$

Diante deste proposição podemos concluir o seguinte lema. 
Lema 5.2.10 Existem constantes $C_{1}, C_{2}>0$ tal que para $n$ e $\ell$ suficientemente grande

$$
1+\frac{C_{1}}{\ell} \leq \frac{\left|d_{n}-c\right|}{\left|z_{n-1}-c\right|}
$$

$e$

$$
\frac{\left|z_{n-1}-c\right|}{\left|z_{n+1}-c\right|} \leq 1+\frac{C_{2}}{\ell}
$$

E segue que $\frac{\left|d_{n}-z_{n-1}\right|}{\left|z_{n-1}-z_{n+1}\right|}$ é limitado longe do 0 .

\section{Demonstração:}

Podemos assumir sem perda de generalidade que $d_{n+3}<z_{n+1}<z_{n-1}<d_{n}$. Assim,

$$
\frac{\left|z_{n-1}-c\right|}{\left|z_{n+1}-c\right|} \leq \frac{\left|d_{n}-c\right|}{\left|d_{n+3}-c\right|}
$$

Usando o fato da aplicação $f$ ter ponto crítico de ordem $\ell$ e a Proposição 5.2 .9 concluimos que

$$
\frac{\left|z_{n-1}-c\right|}{\left|z_{n+1}-c\right|} \leq 1+\frac{C_{2}}{\ell}
$$

$\mathrm{e}$

$$
1+\frac{C_{1}}{\ell} \leq \frac{\left|d_{n}-c\right|}{\left|z_{n-1}-c\right|}
$$

\subsection{Ferramentas Probabilísticas e Ergódicas}

Definição 5.3.1 Dizemos que $(\check{I}, \check{f})$ é uma extensão de Markov (ou torre) de $(I, f)$ se

- (i) I I é uma união enumerável disjunta de intervalos $\check{I}_{j} \subset I$,

- (ii) $\check{f} \check{I}_{j}$ é monótona e $\check{f}\left(\check{I}_{j}\right) \supset \check{I}_{k}$ sempre que $\check{f}\left(\check{I}_{j}\right) \cap \check{I}_{k} \neq \emptyset$,

- $($ iii) $\pi \circ \check{f}=f \circ \pi$, onde $\pi: \check{I} \rightarrow I$ é a projeção natural.

Um exemplo que vamos utilizar neste trabalho é a torre de Hofbauer, que é definido como a seguir: $\check{I} \subset I \times \mathbb{N}$ consiste de uma união enumerável de intervalos $\check{D}_{n}=\left(D_{n}, n\right)$. Os pontos extremos de $D_{n}$ são iterados pra frente de $c$, um dos quais é $c_{n}$. $D_{1}=\left(c_{2}, c_{1}\right)$ e indutivamente

$$
D_{n+1}=\left\{\begin{array}{ccc}
f\left(D_{n}\right) & \text { se } & D_{n} \not \ngtr c, \\
\left(c_{1}, c_{n+1}\right) & \text { se } & D_{n} \ni c .
\end{array}\right.
$$

Sabemos que $c \in D_{n}$ se, e somente se $n=S_{k}$ para algum $k$. Então se $S_{k-1}<n \leq S_{k}$, $D_{n}=\left(c_{n}, c_{n-S_{k-1}}\right) \subset D_{n-S_{k-1}}$. Em particular, $c \in D_{S_{k}} \subset D_{S_{k}-S_{k-1}}=D_{S_{k}^{\prime}}$, para algum tempo de corte $S_{k^{\prime}}<S_{k}$. Além disso, $\check{f}$ age sobre $\check{I}$ como a seguir: Se $(x, n)=\check{x} \in \check{D}_{n}$ então

$$
\check{f}(\check{x})= \begin{cases}(f(x), n+1) & \text { se } x \text { e } c_{n} \text { estão no mesmo lado de } c, \\ \left(f(x), S_{Q(k)}+1\right) & \text { se } x \text { e } c_{n} \text { estão em lados diferentes de } c .\end{cases}
$$

Na primeira situação, dizemos que $\check{x}$ sobe um patamar na torre e no outro caso dizemos que $\check{x}$ tem uma queda na torre e este caso só ocorre quando $n=S_{k}$ é um tempo de corte. Esta observação será muito útil na demonstração do teorema. Além desta observação, segue um lema bastante útil 


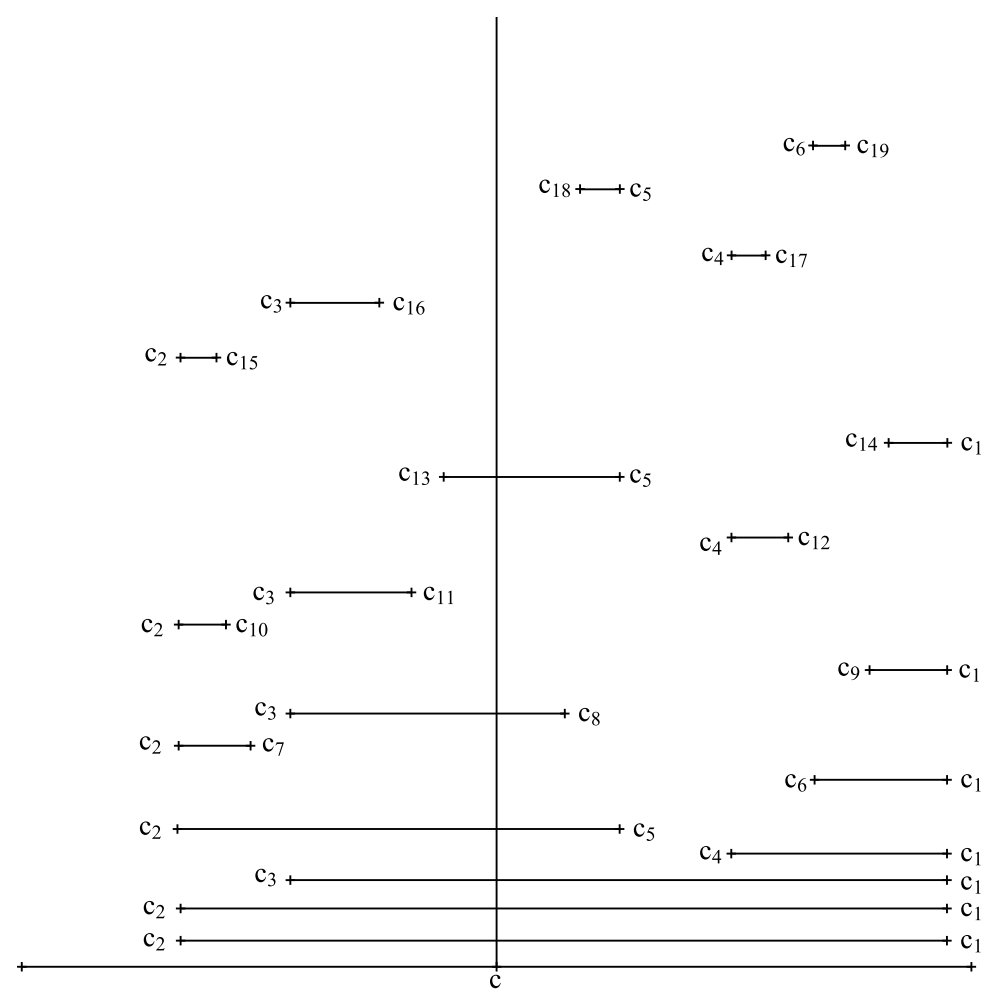

Figura 5.8: A torre para a aplicação de Fibonacci

Lema 5.3.2 Sejam $T=\left(c, d_{n}\right)$ e $t$ algum intervalo que é aplicado monotonicamente em $T$ por $f^{k}$. Sejam $L=\left(c, z_{n+1}\right), J=\left(z_{n+1}, z_{n-1}\right)$ e $R=\left(z_{n-1}, d_{n}\right)$. Sejam l, $j$ e $r$ subintervalos de $t$ que são aplicados por $f^{k}$ em $L, J$ e $R$, respectivamente. Então

$$
\frac{|l|+|r|}{|t|} \geq \frac{3}{4}
$$

Em particular, $\frac{|l|-2|j|+|r|}{t} \geq \frac{1}{4}$.

\section{Demonstração:}

Vimos no lema (5.2.10) que o tamanho de $R$ e $J$ são comparáveis em tamanho, quando $L$ é muito grande. Assim, componha $f^{k}$ com uma transformação de Moebius $M$, que nos forneça intervalos $J$ e $R$ de forma que

$$
|J|=|R|=1
$$

Assim, $|L|$ ainda será grande. Sabemos que $|L| \rightarrow \infty$ quando $\ell \rightarrow \infty$.

Agora, a fim de simplificarmos as contas podemos supor também que $|t|=1$, que pode ser estabelecido por um escalonamento. Podemos chamar o resultado deste escalonamento de $g$, e $\operatorname{assim} \mathcal{S} g=\mathcal{S} f^{k}<0$.

Então $g$ expande a razão cruzada pois $B(g, T, J) \geq 1$ como já vimos na Propriedade (3.2.5). Assim, ficamos com

$$
1 \leq B(g, T, J)=\frac{|T||J|}{|t||j|} \cdot \frac{|l||r|}{|L||R|}
$$


Como $|t|=|J|=|R|=1$ e $|T|=|L|+|J|+|R|$ ficamos com

$$
1 \leq \frac{|L|+2}{|L|} \cdot \frac{|l||r|}{|j|}
$$

Além disso, podemos tomar $L$ suficientemente grande, de forma que

$$
1 \leq \frac{3}{2} \cdot \frac{|l||r|}{|j|}
$$

Sendo assim,

$$
\frac{2}{3}|j| \leq|l||r|
$$

Além disso,

$$
0 \leq(|l|-|r|)^{2}=|l|^{2}-2|l||r|+|r|^{2}
$$

Somando $4|l||r|$ em ambos os lados, obtemos

$$
4|l||r| \leq|l|^{2}+2|l||r|+|r|^{2}=(|l|+|r|)(|l|+|r|)
$$

Como $|t|=|l|+|j|+|r|=1$, ficamos com

$$
4|l||r| \leq(1-|j|)(1-|j|)
$$

De 5.3 e 5.4 , concluimos que

$$
\frac{2}{3}|j| \leq|l||r| \leq\left(\frac{1-|j|}{2}\right)^{2}
$$

Logo, $|j| \leq \frac{3}{8}\left(1-2|j|+|j|^{2}\right)$. Resolvendo esta inequação do segundo grau, concluimos que ou $|j| \leq \frac{1}{3}(7-2 \sqrt{10})$ ou $|j| \geq \frac{1}{3}(7+2 \sqrt{10})$. E como por hipótese $1=|t|>|j|$ concluímos que

$$
|j| \leq \frac{1}{3}(7-2 \sqrt{10})=0,2251482265544137 \ldots
$$

E em particular, como

$$
\frac{|l|+|r|}{|t|}=\frac{1-|j|}{|t|}=1-|j| \geq 1-0,25=\frac{3}{4}
$$

$\mathrm{E}|j| \leq \frac{1}{4}$ podemos concluir que

$$
\frac{|l|+|r|-2|j|}{|t|} \geq \frac{1}{4}
$$

Lema 5.3.3 Seja $y=\left\{y_{i}\right\}_{i \geq 1}$ uma sequência estocástica tomando valores em $\{-1,1\}$, e seja $\left\{T_{n}\right\}_{n}$ as somas parciais, ou seja, $T_{n}=\sum_{j=1}^{n} y_{j}$. Se para algum $p \in[0,1]$,

$$
P\left\{y_{k}=1 \mid y_{1}, \cdots, y_{k-1}\right\} \geq p,
$$

para todo $k$ e $y_{1}, \cdots, y_{k-1}$, então

$$
\liminf _{n} \frac{1}{n} T_{n} \geq \mathbb{E}(Y)=2 p-1 \text { q.c. }
$$

\section{Demonstração:}

Ver [Bru94b]. 
Como veremos a seguir, não é essencial que $y_{i}$ sejam valores apenas em $\{-1,1\}$. Assim, este lema não será usado diretamente em nenhum momento, porém foi importante para se construir a Proposição 5.3.5.

Teorema 5.3.4 Sejam $\mathcal{F}_{1}$ e $\mathcal{F}_{2} \sigma$-álgebras. Se $\mathcal{F}_{1} \subset \mathcal{F}_{2}$ então

(i) $\mathbb{E}\left(\mathbb{E}\left(X \mid \mathcal{F}_{1}\right) \mid \mathcal{F}_{2}\right)=\mathbb{E}\left(X \mid \mathcal{F}_{1}\right)$

(ii) $\mathbb{E}\left(\mathbb{E}\left(X \mid \mathcal{F}_{2}\right) \mid \mathcal{F}_{1}\right)=\mathbb{E}\left(X \mid \mathcal{F}_{1}\right)$.

\section{Demonstração:}

Ver [Dur10].

Proposição 5.3.5 Seja $y=\left\{y_{i}\right\}_{i \geq 1}$ uma sequência estocástica tomando valores em $\{-2,-1,1\}$, e seja $\left\{T_{n}\right\}_{n}$ as somas parciais, ou seja, $T_{n}=\sum_{j=1}^{n} y_{j}$. Se para algum $p \in[0,1]$,

$$
P\left\{y_{k}=1 \mid y_{1}, \cdots, y_{k-1}\right\} \geq p,
$$

para todo $k$ e $y_{1}, \cdots, y_{k-1}$, então

$$
\liminf _{n} \frac{1}{n} T_{n} \geq 3 p-2 \quad q . c .
$$

\section{Demonstração:}

Pelo lema 5.3.3, observamos inicialmente que o resultado será válido no caso em que $y=\left\{y_{i}\right\}$ for uma sequência estocástica tomando valores em $\{-2,1\}$. Neste caso, o $\lim _{n} \inf \frac{T_{n}}{n}$ é limitado pela esperança da variável aleatória $Y$ de distribuição

$$
P\left\{y_{k}=1 \mid y_{1}, \cdots, y_{k-1}\right\}=p \text { e } P\left\{y_{k}=-2 \mid y_{1}, \cdots, y_{k-1}\right\}=1-p
$$

devido ao fato da esperança ser linear. Assim,

$$
\mathbb{E}\left\{y_{n} \mid y_{1}, \cdots, y_{n-1}\right\}=1 \cdot p+(-2)(1-p)=3 p-2
$$

Assim,

$$
\lim _{n} \inf \frac{1}{n} T_{n} \geq \mathbb{E}(Y)=3 p-2 \text { q.c. }
$$

Definamos agora

$$
z_{n}=\mathbb{1}_{\left[y_{n}=1\right]}-2 \mathbb{1}_{\left[y_{n}=-1\right] \cup\left[y_{n}=-2\right]} \quad \forall n
$$

Sendo assim, temos $\sigma\left(z_{1}, \cdots, z_{n}\right) \subseteq \sigma\left(y_{1}, \cdots, y_{n}\right)$ para todo $n$. Logo,

$$
\begin{aligned}
P\left\{z_{n}=1 \mid z_{1}, \cdots, z_{n-1}\right\} & =\mathbb{E}\left\{\mathbb{1}_{\left[z_{n}=1\right]} \mid z_{1}, \cdots, z_{n-1}\right\} \\
& =\mathbb{E}\left\{\mathbb{E}\left\{\mathbb{1}_{\left[z_{n}=1\right]} \mid z_{1}, \cdots, z_{n-1}\right\} \mid y_{1}, \cdots, y_{n-1}\right\}
\end{aligned}
$$

Pelo teorema 5.3.4 obtemos

$$
\begin{aligned}
\mathbb{E}\left\{\mathbb{E}\left\{\mathbb{1}_{\left[z_{n}=1\right]} \mid z_{1}, \cdots, z_{n-1}\right\} \mid y_{1}, \cdots, y_{n-1}\right\} & =\mathbb{E}\left\{\mathbb{E}\left\{\mathbb{1}_{\left[z_{n}=1\right]} \mid y_{1}, \cdots, y_{n-1}\right\} \mid z_{1}, \cdots, z_{n-1}\right\} \\
& =\mathbb{E}\left\{\mathbb{E}\left\{\mathbb{1}_{\left[y_{n}=1\right]} \mid y_{1}, \cdots, y_{n-1}\right\} \mid z_{1}, \cdots, z_{n-1}\right\} \\
& =P\left\{y_{n}=1 \mid y_{1}, \cdots, y_{n-1}\right\} \geq p
\end{aligned}
$$


Como já sabemos que

$$
\lim _{n} \inf \frac{z_{1}+\cdots+z_{n}}{n} \geq 3 p-2
$$

e além disso $z_{n} \leq y_{n}$ a.s. Portanto, concluímos que

$$
\liminf _{n} \frac{y_{1}+\cdots+y_{n}}{n} \geq 3 p-2
$$

Além disso, $P\left\{y_{n}=1 \mid y_{1}, \cdots, y_{n-1}\right\} \geq p$ só precisa ser verdadeiro para estados suficientemente altos no passeio aleatório para utilizarmos na proposição a seguir.

Sendo assim, segue a proposição imprescindível na demonstração do teorema.

Proposição 5.3.6 Para cada $N \in \mathbb{N}$ e todo $n>S_{N}$, existem conjuntos de medida positiva $\check{B}$ na torre tal que todo $\check{x} \in \check{B}$, \#\{orb $\left.(x) \cap \cup_{m \leq S_{N}} \check{D}_{m}\right\}<\infty$.

\section{Demonstração:}

Vamos considerar a ação de $f$ na torre como um passeio aleatório, que iremos estimar por baixo por uma estacionário. Este passeio aleatório será nos inteiros não negativos. Ao invés de considerarmos os níveis de tempo de corte $D_{S_{k}}$, vamos considerar a parte $\check{E}_{S_{k}}=\left(c, \check{d}_{k}\right) \subset \check{D}_{S_{k}}$.

Seja $\check{E}=\cup_{i \geq 0} \check{E}_{S_{i}}$. Para $k \geq 2, \check{E}_{S_{k}}$ pode ser dividido em quatro partes, como segue

$$
\left(\check{c}, \check{d}_{k}\right)=\left(\check{c}, \check{z}_{k+1}\right) \cup\left(\check{z}_{k+1}, \check{z}_{k}\right) \cup\left(\check{z}_{k}, \check{z}_{k-1}\right) \cup\left(\check{z}_{k-1}, \check{d}_{k}\right)
$$

De forma que

- $\left(\check{c}, \check{z}_{k+1}\right), \check{F}\left(\check{c}, \check{z}_{k+1}\right)=\check{f}^{S_{k+1}}\left(\check{c}, \check{z}_{k+1}\right)=\check{E}_{S_{k+1}}$

- $\left(\check{z}_{k-1}, \check{d}_{k}\right), \check{F}\left(\check{z}_{k-1}, \check{d}_{k}\right)=\check{f}^{S_{k-1}}\left(\check{z}_{k-1}, \check{d}_{k}\right)=\check{E}_{S_{k+1}}$

- $\left(\check{z}_{k+1}, \check{z}_{k}\right), \check{F}\left(\check{z}_{k+1}, \check{z}_{k}\right)=\check{f}^{S_{k+1}}\left(\check{z}_{k+1}, \check{z}_{k}\right)=\check{E}_{S_{k-1}}$

- $\left(\check{z}_{k}, \check{z}_{k-1}\right), \check{F}\left(\check{z}_{k}, \check{z}_{k-1}\right)=\check{f}^{S_{k}}\left(\check{z}_{k}, \check{z}_{k-1}\right)=\check{E}_{S_{k-2}}$

Esta partição define uma partição enumerável de Markov de $\check{E}$. A aplicação correspondente $\check{F}$ é uma aplicação induzida de $\check{f}$, sendo esta a aplicação de primeiro retorno de $\check{E}$ sob uma iteração de $\check{F}$. Os pontos podem ou subir um nível ou cair um ou dois níveis.

Seja $T_{n}(\check{x})=k$. Se $F^{n}(x) \in \check{E}_{S_{k}}$ então

$$
T_{n}(\check{x})=T_{0}(\check{x})+y_{1}(\check{x})+y_{2}(\check{x})+\cdots+y_{n}(\check{x})
$$

onde $y_{j}(\check{x}) \in\{-2,-1,1\}$ conforme $\check{F}^{j-1}$ sobe um nível ou cai um ou dois níveis na próxima iteração de $\check{F}$.

Vimos no lema 5.2.2 que $\left|\check{E}_{S_{i}}\right|$ decresce exponencialmente, assim $\sum_{i}\left|\check{E}_{S_{i}}\right|<\infty$ e a medida de Lebesgue pode ser normalizada. Portanto, considere $\check{E}$ um espaço de probabilidade, tendo a medida de Lebesgue normalizada como a medida de probabilidade.

Por definição de probabilidade condicional,

$$
P\left\{y_{n}(\check{x})=1 \mid T_{n-1}(\check{x})>N\right\}=\frac{\left|\left\{\check{x} \in \check{E} \mid y_{n}(\check{x})=1 \mathrm{e} T_{n-1}(\check{x})>N\right\}\right|}{\left|\left\{\check{x} \in \check{E} \mid T_{n-1}(\check{x})>N\right\}\right|}
$$

Como $\check{F}$ é uma aplicação de Markov, $\check{E}$ é uma união enumerável de intervalos dois a dois disjuntos $\check{J}$ com a propriedade que a aplicação $\check{F}^{n-1}$ leva $\check{J}$ monotonicamente em $\check{E}_{S_{k}}$ para algum $k \geq 0$. 
Assim, cada intervalo $\check{J}$ pode ser escrito como a união disjunta de dois intervalos

$$
\check{J}=\check{J}_{\text {cima }} \cup \check{J}_{\text {baixo }}
$$

Em que

- $\check{F}^{n-1}\left(\check{J}_{\text {cima }}\right)=\left(\check{c}, \check{z}_{k+1}\right) \cup\left(\check{z}_{k-1}, \check{d}_{k}\right)$

- $\check{F}^{n-1}\left(\check{J}_{\text {baixo }}\right)=\left(\check{z}_{k+1}, \check{z}_{k-1}\right)$

Assim, se $k>N$, então pelo lema 5.3.2 temos $\frac{\left|\breve{J}_{\text {cima }}\right|}{|\tilde{J}|} \geq \frac{3}{4}$ e $\frac{\left|\check{J}_{\text {baixo }}\right|}{|\check{J}|} \leq \frac{1}{4}$.

A órbita $\check{F}$ do intervalo $\check{J}$ é determinada pelo seu nível de partida, chamemos $T_{0}$, e das etapas sucessivas $y_{1}, \cdots, y_{n-1}$.

Somando sobre todos os intervalos $\check{J}$ tendo uma determinada órbita $\check{F}$ tal que $k>N$, temos

$$
\frac{\mid\left\{\check{x} \in \check{E} \mid y_{i}(\check{x})=y_{i} \text { para } i<n, y_{n}(\check{x})=1 \text { e } T_{n-1}(\check{x})>N\right\} \mid}{\mid\left\{\check{x} \in \check{E} \mid y_{i}(\check{x})=y_{i} \text { para } i<n \text { e } T_{n-1}(\check{x})>N\right\} \mid} \geq \frac{3}{4}
$$

Assim, $P\left\{y_{n}=1 \mid T_{0}, y_{1}, \cdots, y_{n-1}, T_{n-1}>N\right\} \geq \frac{3}{4}$. Devido ao fato de $y_{n} \in\{-2,-1,1\}$ e como $\left|\check{E}_{S_{i}}\right|$ decresce exponencialmente e $P\left\{T_{0}=k\right\}=\frac{\left|\check{E}_{S_{k}}\right|}{|\check{E}|}$, percebemos que podemos aplicar a proposição 5.3.5 e assim concluímos que existe um conjunto de medida positiva de pontos que sobem na torre.

\subsection{Demonstração do Teorema B}

Após construirmos com bastante cuidado e rigor todas as ferramentas necessárias para a demonstração é preciso estabelecer alguns fatos básicos sobre as medidas invariantes e absolutamente contínuas na torre antes de iniciarmos a prova.

Chamaremos uma aplicação de ergódica com respeito à medida de Lebesgue se para cada conjunto $A$ completamente invariante, temos ou $|A|=0$ ou $\left|A^{c}\right|=0$. Chamaremos a aplicação $f$ de conservativa se para cada conjunto invariante pra frente temos ou $|A|=0$ ou $\left|A^{c}\right|=0$ (e também para os conjuntos invariantes para trás) $A$. Notemos que este é um conceito mais forte do que o usual na teoria ergódiga pois a definição standard nos diz que $f$ é conservativa com respeito à medida de Lebesgue se não existe um conjunto de medida positiva tal que $f^{n}(A) \cap A=\emptyset$ para todo $n$. Se $f$ não for conservativa então ela será chamada de dissipativa.

Exemplo 5.4.1 Seja $f:[0,1] \rightarrow[0,1]$ definida por $f(x)=x$. Segundo a definição usual na teoria ergódica temos $f$ conservativa, contudo segundo a definiçẫo que usaremos aqui temos $f$ dissipativa.

Além disso, vamos invocar o seguinte resultado

Teorema 5.4.2 Seja $f$ uma aplicação S-unimodal, então $(I, f)$ é ergódico (conservativo) se, e somente se, $(\check{I}, \check{f})$ é ergódico (conservativo).

\section{Demonstração:}

Em [Bru94b].

Teorema B. Se $g_{\gamma}$ é aplicação de Fibonacci e $\ell$ é suficientemente grande, o conjunto $\omega$-limite do seu ponto crítico é um conjunto de Cantor minimal de medida Lebesgue nula cuja bacia tem medida de Lebesgue total. 
Seja $\check{B}=\check{B}(n)$ um conjunto de medida positiva na torre como vimos na proposição 5.3.6. Seja $\check{B}_{0}=\bigcup_{j \geq 0} \bigcup_{i \geq 0} f^{-i} \circ f^{j}(\check{B})$. Desta forma sabemos que $\check{B}_{0}$ é completamente invariante e, por ergodicidade, sabemos que possui medida total.

Para cada $n, \check{B}_{0}=\check{B}_{0}(n)$ consiste de pontos que visitam $\bigcup_{j \leq n} \check{D}_{j}$ apenas um número finito de vezes. Assim, temos $\check{f}$ não conservativo. O conjunto $\check{X}=\left\{\check{x} \in \check{I} \mid \check{f}^{n}(\check{x}) \notin \check{D}_{1}\right.$, para todo $\left.n \in \mathbb{N}\right\}$ é um exemplo de um conjunto invariante para frente com $|\check{X}|>0$ e $\left|\check{X}^{c}\right|>0$.

Agora, aplicando o teorema 5.4.2 concluimos que $f$ também não é conservativo.

Usando também o fato de que podemos escrever

$$
\omega(c)=\bigcap_{i} \bigcup_{j \geq i} \bar{D}_{j}
$$

Conforme [ML93], podemos ser mais preciso e obtermos

$$
\omega(c) \subset C_{k}=\left\{\bigcup_{S_{k}<i \leq S_{k+1}} D_{i} \cup \bigcup_{S_{k+1}+S_{k-1}<i \leq S_{k+2}} D_{i}\right\}
$$

Seja $U$ uma vizinhança arbitrária de $\omega(c)$. Como $\left|D_{i}\right| \rightarrow 0$ quando $i \rightarrow \infty$, existe um $k_{0}$ tal que para todo $k>k_{0}$ temos

$$
\omega(c) \subset C_{k} \subset U
$$

Então temos $\omega(x) \subset \omega(c)$ q.t.p. Concluímos então a prova.

A prova original deste resultado pode ser encontrado em [Bru94b] e em [BKNvS96]. 


\section{Referências Bibliográficas}

[BB04] Henk Bruin e Karen Brucks. Topics from One-dimensional dynamics, volume 62. London Mathematical Society, 2004. 11

[BH92] B. Branner e J. H. Hubbard. The iteration of cubic polynomials, part ii: Patterns and parapatterns. Acta Math., 169:229-325, 1992. 41

[BKNvS96] H. Bruin, G. Keller, T. Nowicki e S. van Strien. Wild cantor attractors exist. Ann. Math., 143:97-130, 1996. 3, 42, 59

[Bru94a] H. Bruin. Combinatorics of the kneading map. Intern. J. Bifur. and Chaos, 5:13391349, 1994. 10, 36

[Bru94b] H. Bruin. Invariant Measures for interval maps. Tese de Doutorado, Universidade de Delft, Holanda, Junho 1994. 55, 58, 59

[Bru98] H. Bruin. The existence of absolutely continuous invariant measures is not a topological invariant for unimodal maps. Ergodic theory and dynamical systems, 18:555-565, 1998. 2,40

[dMvS93] W. de Melo e S. van Strien. One-dimensional dynamics. Springer Verlag, 1993. 20, 21, 36

[Dur10] Rick Durrett. Probability: Theory and Examples. Cambridge U. Press, 2010. 56

[GJ90] J. Guckenheimer e S. Johnson. Distortion of s-unimodal maps. Annals of Mathematics, 132:71-130, 1990. 41

[GS97] J. Graczyk e G. Swiatek. Generic hyperbolicity in the logistic family. Ann. Math., 146:1-52, 1997. 37

[Guc79] J. Guckenheimer. Sensitive dependence on initial conditions for unimodal maps. Commun. Math. Phys., 70:133-160, 1979. 41

[HK90] F. Hofbauer e G. Keller. Quadratic maps without asymptotic measure. Communications in Mathematical Physics, 127:319-337, 1990. 8

[LB86] M. Lyubich e A. M. Blokh. Attractors of the interval. Banach Center Publ., 23:427-442, 1986. 41

[Lyu91] M. Lyubich. Ergodic theory for smooth one dimensional dynamical systems. 1991. 41

[ML93] J. Milnor e M. Lyubich. The fibonacci unimodal map. J. Amer. Math. Soc., 6:425-457, 1993. 41,59

[MT88] J. Milnor e W. Thurston. On iterated maps of the interval. Dynamical Systems, 18:465-563, 1988. 5, 6

[Sin78] D. Singer. Stable orbits and bifurcation of maps of the interval. SIAM J. Appl. Math., 35:260-267, 1978. 13 
[Tsu85] I. Tsuda. On the abnormality of period doubling bifurcations. Progress Theor. Phys., $66: 1985-2002,1985.42$

[Yoc] J.-C. Yoccoz. Il n'y a pas de contre-exemple de denjoy analytiques. 41 